\title{
An overview of current issues in the uptake of atmospheric trace gases by aerosols and clouds
}

C. E. Kolb ${ }^{1}$, R. A. Cox ${ }^{2}$, J. P. D. Abbatt ${ }^{3}$, M. Ammann ${ }^{4}$, E. J. Davis ${ }^{5}$, D. J. Donaldson ${ }^{3}$, B. C. Garrett ${ }^{6}$, C. George ${ }^{7}$, P. T. Griffiths ${ }^{2}$, D. R. Hanson ${ }^{8}$, M. Kulmala ${ }^{9}$, G. McFiggans ${ }^{10}$, U. Pöschl ${ }^{11}$, I. Riipinen ${ }^{9}$, M. J. Rossi ${ }^{12}$, Y. Rudich ${ }^{13}$, P. E. Wagner $^{14}$, P. M. Winkler ${ }^{14,15}$, D. R. Worsnop ${ }^{1,9}$, and C. D. O' Dowd ${ }^{16}$

${ }^{1}$ Center for Aerosol and Cloud Chemistry, Aerodyne Research, Inc., 45 Manning Road, Billerica, MA 01821-3976, USA

${ }^{2}$ Centre for Atmospheric Science, Department of Chemistry, University of Cambridge, Lensfield Road, Cambridge CB2 1EP, UK

${ }^{3}$ Department of Chemistry, University of Toronto, 80 St. George Street, Toronto, ON, M5S 3H6, Canada

${ }^{4}$ Laboratory of Radiochemistry and Environmental Chemistry, Paul Scherrer Institute, 5232 Villigen, Switzerland

${ }^{5}$ Department of Chemical Engineering, University of Washington, Seattle, WA 98195-1750, USA

${ }^{6}$ Fundamental \& Computational Sciences Directorate, Pacific Northwest National Laboratory, Richland, WA 99352, USA

${ }^{7}$ IRCELYON, Institut de recherches sur la catalyse et l'environnement de Lyon, CNRS UMR 5256, Université Lyon 1. 2, Av. Albert Einstein, 69626 Villeurbanne Cedex, France

${ }^{8}$ Augsburg College, Minneapolis, MN 55454, USA

${ }^{9}$ Department of Physical Sciences, University of Helsinki, P.O. Box 64, 00014 Helsinki, Finland

${ }^{10}$ Centre for Atmospheric Sciences, School of Earth, Atmospheric \& Environmental Sciences, University of Manchester, Manchester, M13 9PL, UK

${ }^{11}$ Biogeochemistry Department, Max Planck Institute for Chemistry, 55128 Mainz, Germany

${ }^{12}$ Laboratorium fuer Atmosphaerenchemie, OFLA008, Paul Scherrer Institute, 5232 Villigen, Switzerland

${ }^{13}$ Department of Environmental Sciences, Weizmann Institute, Rehovot 76100, Israel

${ }^{14}$ Faculty of Physics, University of Vienna, 1090 Vienna, Austria

${ }^{15}$ Atmospheric Chemistry Division, National Center for Atmospheric Research, Boulder, CO 80305-5602, USA

${ }^{16}$ School of Physics and Centre for Climate \& Air Pollution Studies, National University of Ireland Galway, University Road, Galway, Ireland

Received: 9 April 2010 - Published in Atmos. Chem. Phys. Discuss.: 27 April 2010

Revised: 7 October 2010 - Accepted: 11 October 2010 - Published: 10 November 2010

\begin{abstract}
A workshop was held in the framework of the ACCENT (Atmospheric Composition Change - a European Network) Joint Research Programme on "Aerosols" and the Programme on "Access to Laboratory Data". The aim of the workshop was to hold "Gordon Conference" type discussion covering accommodation and reactive uptake of water vapour and trace pollutant gases on condensed phase atmospheric materials. The scope was to review and define the current state of knowledge of accommodation coefficients for water vapour on water droplet and ice surfaces, and uptake of trace gas species on a variety of different surfaces characteristic of the atmospheric condensed phase particulate matter and cloud droplets. Twenty-six scientists participated in this
\end{abstract}

meeting through presentations, discussions and the development of a consensus review.

In this review we present an analysis of the state of knowledge on the thermal and mass accommodation coefficient for water vapour on aqueous droplets and ice and a survey of current state-of the-art of reactive uptake of trace gases on a range of liquid and solid atmospheric droplets and particles. The review recommends consistent definitions of the various parameters that are needed for quantitative representation of the range of gas/condensed surface kinetic processes important for the atmosphere and identifies topics that require additional research.

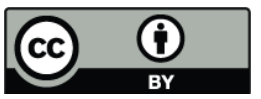

Correspondence to: C. E. Kolb

(kolb@aerodyne.com)

Published by Copernicus Publications on behalf of the European Geosciences Union. 


\section{Introduction and motivation}

\subsection{Why are trace gas uptake processes important?}

Trace gas uptake by a variety of condensed phase materials on the Earth's surface, including vegetation, rock, soil, ice, snow, fresh and marine surface waters, buildings and paved surfaces can play an important role in the transformation and environmental fate of many atmospheric species. Even more importantly, heterogeneous interactions of trace gases with liquid and solid cloud droplets and aerosol particulate matter (PM) strongly influence two critical atmospheric properties.

First, heterogeneous uptake by PM and cloud droplets, often coupled with surface and/or bulk phase reactions, can significantly alter the distribution of reactive atmospheric gases. Heterogeneous processes change the gas phase concentrations of photochemical oxidants, acid gases, free radicals, and a wide variety of soluble and/or semi-volatile species, significantly impacting important atmospheric processes and phenomena including photochemical smog, acid deposition, tropospheric oxidative cleansing, reactive greenhouse gas concentrations, and stratospheric ozone depletion.

Second, the uptake of trace gases, including water vapour, impacts important physical properties of atmospheric PM, such as size, optical properties, and ability to nucleate cloud droplets. These properties all impact the direct interaction of PM with atmospheric radiation or their ability to alter cloud formation and evaporation rates, which also influence atmospheric radiative fluxes as well as precipitation patterns. Thus, trace gas uptake processes must be understood in order to characterize the impact of atmospheric PM on the current climate and to predict its influence on the future's climate.

The following discussion will focus on the interaction of trace gases with atmospheric cloud droplets and PM, although some of the material is also pertinent to trace gas interactions with materials at the Earth's surface.

\subsection{Who needs to know about heterogeneous trace gas uptake?}

Nearly all atmospheric scientists concerned with atmospheric chemistry, aerosol microphysics or atmospheric radiative transport need to be concerned with trace gas uptake processes. As noted above these processes can have a significant impact on both the reactive trace gas distribution and the size distribution and character of aerosol PM.

Both atmospheric chemistry and climate modelers need to adequately compute the trace gas and PM content of the atmosphere and, therefore need to adequately represent trace gas uptake processes in both their diagnostic and prognostic models. Laboratory scientists quantifying atmospheric chemistry and physics processes often must account for trace gas uptake processes by deliberatively or accidentally entrained PM, as well as by the walls of their apparatus. Field measurement studies come with their own component of cloud droplets and/or aerosol PM that interact with the ambient trace gases and often influence the atmospheric properties being studied.

In recognition of these needs the topic of heterogeneous processes involving atmospheric trace gases has been included in the evaluations carried out by the NASA-JPL data evaluation panel. In their most recent full evaluation (Evaluation 15, July 2006; http://jpldataeval.jpl.nasa.gov/download. html) heterogeneous reactions in the stratosphere and selected heterogeneous processes in the free troposphere are addressed; an updated evaluation is scheduled for publication in late 2010. Recently the IUPAC Subcommittee for kinetics data evaluation for atmospheric chemistry has undertaken an evaluation of data for heterogeneous reactions which is presented on the IUPAC website at University of Cambridge, UK. (http://www.iupac-kinetic.ch.cam.ac.uk/) and has been partially published in this journal (Crowley et al., 2010). A further publication of evaluated data for uptake on liquid surfaces will be submitted to ACPD soon.

\subsection{What are the relevant atmospheric trace gas uptake surfaces?}

Atmospheric aerosol particles and cloud/fog droplets often have complex chemical compositions and some may occur in both liquid and solid phases, depending on ambient atmospheric conditions. Aqueous droplets may change from liquid to ice depending on atmospheric temperature, while aqueous salt PM may cycle between liquid and crystalline phases depending on relative humidity $(\mathrm{RH})$.

Some primary PM particles are usually emitted as solids, including entrained dust and soot from combustion, while others such as sea salt particles released by wave action, and sulphuric acid produced by burning sulphur containing fuels may initially form liquid droplets.

Atmospheric PM evolves by serving as heterogeneous condensation sites for semi-volatile inorganic and organic species emitted from various sources or created by atmospheric chemical processes, including inorganic and organic acids that can form salts by co-condensing with atmospheric ammonia or reacting with labile cations (e.g. $\mathrm{Na}^{+}, \mathrm{K}^{+}$, $\mathrm{Ca}^{++}$) in primary PM. It can also evolve by heterogeneous or (for liquids) bulk phase chemical or photochemical reactions with atmospheric oxidants, by agglomeration with dissimilar PM, or through "cloud processing" when absorbed into a cloud/fog droplet that later evaporates, mixing scavenged low volatility components.

Finally, recent advances in measuring the chemical content of atmospheric PM have revealed that submicron fine PM typically has a very significant (20-80\%) organic component, usually composed of a very large number of individual compounds. These organic species may be highly surface active, forming surface layers on aqueous or solid particles that may strongly affect trace gas uptake properties; they may 
also form separate hydrophobic organic phases within aqueous PM droplets.

The wide diversity of atmospheric PM surfaces poses a major challenge for both experimental and theoretical trace gas uptake studies. Most studies have been preformed on model systems that are composed of single or relatively few components. Studies that systematically vary multiple component surfaces are necessary and are starting to be more common.

\section{Processes, models and underlying physical chemistry}

\subsection{Multiple interacting physical and chemical processes}

The interaction of a trace gas molecule with the surface of a droplet or particle involves multiple transport and kinetic processes, including gas phase diffusion to the surface, thermal accommodation with the surface, adsorption to and desorption from the surface, possible reaction at the gas/surface interface, and, for liquids and some amorphous solid surfaces, interfacial mass transport of trace species (mass accommodation), solvation, bulk phase diffusion and bulk phase reaction (Kolb et al., 1995).

Figure 1 provides a simple schematic illustration of key processes and flux terms used to describe atmospheric gas uptake by atmospheric particles: gas kinetic flux of surface collisions $\left(J_{\text {coll }}\right)$, the adsorption onto the particle surface $\left(J_{\mathrm{ads}}\right)$, absorption into the particle bulk $\left(J_{\mathrm{abs}}\right)$, desorption from the surface $\left(J_{\mathrm{des}}\right)$, and net uptake by the condensed phase $\left(J_{\text {net }}\right)$. The red arrows indicate production and loss of chemical species by reactions at the particle surface $\left(P_{\mathrm{s}}\right.$, $\left.L_{\mathrm{S}}\right)$ or in the particle bulk $\left(P_{\mathrm{b}}, L_{\mathrm{b}}\right)$. Note that further refinements in the representation of the gas-particle interface region are needed to resolve interactions of multiple chemical species and reactive processes, especially at the surface of solid or highly viscous liquid particles (e.g.: distinction of sorption and quasi-static surface molecular layers for the description of Langmuir-Hinshelwood- or Eley-Rideal-type reactions) (Pöschl et al., 2007; Ammann and Pöschl, 2007).

\subsection{Definitions and terminology}

In atmospheric science, the terms mass accommodation and thermal accommodation are generally used to describe the transfer of chemical species (molecules, radicals, atoms) and thermal energy from the gas phase to the condensed phase (aerosol or cloud particles).

In the scientific literature, different rate parameters and formalisms have been defined and used for the quantitative mathematical description and modelling of these processes. Unfortunately, the terminology used in these descriptions has varied significantly, particularly among relevant sub-fields in atmospheric chemistry, materials science, physical chemistry and aerosol and cloud physics. Indeed, various subfields use

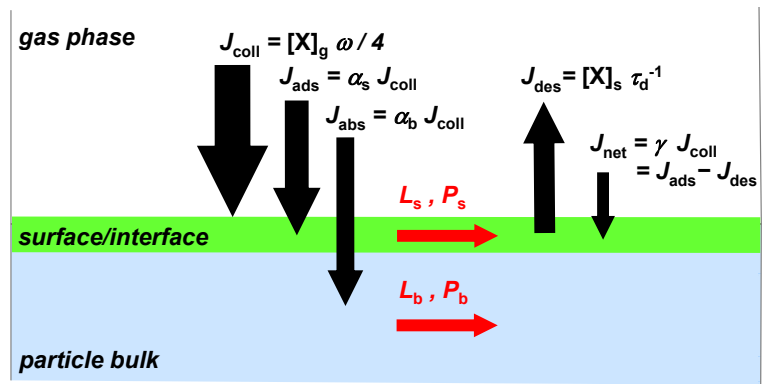

Fig. 1. Schematic illustration of key processes and flux terms describing gas uptake by atmospheric particles: gas kinetic flux of surface collisions $\left(J_{\text {coll }}\right)$, the adsorption onto the particle surface $\left(J_{\text {ads }}\right)$, absorption into the particle bulk $\left(J_{\text {abs }}\right)$, desorption from the surface $\left(J_{\mathrm{des}}\right)$, and net uptake by the condensed phase $\left(J_{\text {net }}\right)$. The red arrows indicate production and loss of chemical species by reactions at the particle surface $\left(P_{\mathrm{s}}, L_{\mathrm{S}}\right)$ or in the particle bulk $\left(P_{\mathrm{b}}\right.$, $\left.L_{\mathrm{b}}\right) .[\mathrm{X}]_{\mathrm{g}}$ and $[\mathrm{X}]_{\mathrm{S}}$ stand for the gas and surface concentrations of volatile species $\mathrm{X} ; \omega$ is the mean free path of $\mathrm{X}$ in the gas phase; $\alpha_{\mathrm{S}}$ and $\alpha_{\mathrm{b}}$ are the surface and bulk accommodation coefficients; $\tau_{\mathrm{d}}$ is the desorption lifetime, and $\gamma$ is the net uptake coefficient (Pöschl et al., 2007).

different terms for the same process or the same term for differing processes.

In this section, we propose a consistent terminology and use of symbols to describe the different steps in the uptake process. These definitions are accompanied by some key questions and exemplary references to the scientific literature.

1. Thermal accommodation (energy transfer): Gas molecules collide with the surface and equilibrate with the surface in all degrees of freedom (kinetic and internal energy), typically on a time scale of $\sim 10^{-11} \mathrm{~s}$. The thermal accommodation coefficient is the number of molecules equilibrated with the surface divided by the total number of molecules colliding with the surface. Literature symbols have included: $\alpha_{t}$ and $\mathrm{S}$ (Winkler et al., 2004; Vieceli et al., 2005). We recommend the use of $\alpha_{t}$.

2. Surface accommodation (adsorption): Gas molecules may collide with a surface and not be immediately reflected, staying at the surface for longer than the duration of direct scattering processes $\left(>10^{-12} \mathrm{~s}\right)$. Unlike thermal accommodation, surface accommodated molecules may retain part of their collisional kinetic energy. The accommodated or adsorbed molecule is bound to the surface by relatively weak forces, such as van der Waals forces or hydrogen bonds. The surface science literature refers to this process as physisorption or trapping. The surface accommodation coefficient is the number of molecules accommodated at the surface divided by the total number of molecules colliding with 
the surface. Literature symbols have included: $\alpha^{\prime}, \alpha_{\mathrm{s}}$ and S (Jayne et al., 1990; Hanson, 1997a; Ammann et al., 2003; Vieceli et al., 2005; Garrett et al., 2006; Ammann and Pöschl, 2007; Pöschl et al., 2007). In the surface science literature surface accommodation or adsorption often is not clearly distinguished from processes leading to chemisorption, which typically refer to surface species with significant alteration of their gas phase electronic structure and which may even include dissociation. Thus, surface accommodation often is not equivalent to the sticking coefficient used in surface science to describe both physisorptive and chemisorptive interactions (e.g. King and Wells, 1972). We recommend the use of surface accommodation to designate physisorptive processes, with the definition given above and the symbol $\alpha_{\mathrm{s}}$, to clearly separate it from subsequent chemisorptive processes.

3. Bulk accommodation (absorption): After surface accommodation gas molecules may be incorporated into the bulk of the condensed phase by interfacial mass transport processes involving their solvation in the interface region. Bulk accommodation is therefore a complex process. The bulk accommodation coefficient is the number of molecules incorporated into the particle bulk divided by the total number of molecules colliding with the surface. Note that for liquid surfaces the bulk accommodation coefficient is often described as the mass accommodation coefficient, usually designated as $\alpha$ in much of the literature cited in this review and in discussions in subsequent sections below. Literature symbols for bulk accommodation have included: $\alpha, \alpha_{m}$, and $\alpha_{b}$ (Jayne et al., 1990; Hanson, 1997a; Ammann et al., 2003; Winkler et al., 2004; Ammann and Pöschl, 2007; Pöschl et al. 2007). We recommend the use of the symbol $\alpha_{b}$ that designates the physical state of the accommodated species (dissolved in the bulk).

4. Net gas uptake (experimentally derived quantities): The uptake coefficient for a trace gas is defined as the ratio of the number of trace gas molecules removed from the gas phase (net loss) divided by the total number of trace gas collisions with the surface. This net uptake is the result of the accommodation processes described above and subsequent processes such as surface reaction, bulk diffusion and reaction, if applicable. The uptake coefficient may therefore be a time and concentration dependent quantity. It is often difficult to experimentally determine which, if any, of the accommodation coefficients defined above are actually controlling the heterogeneous uptake of trace gas species in either laboratory experiments or the atmosphere. Most measurements determine the rate of loss of trace gas phase species and/or the rate of gain of condensed phase trace species to or through a known or estimated liquid or solid surface area. The uncorrected measured uptake coefficient is often designated $\gamma$ or $\gamma_{\text {meas }}$, while the experimentally determined uptake coefficient corrected for, e.g., gas phase diffusion effects is often designated $\gamma_{0}$ (Davidovits et al. (2006) and references therein). On the other hand, the uptake coefficient may be time dependent due to changing surface properties, so many studies use $\gamma_{0}$ to designate the initial uptake coefficient observed at the earliest times during an uptake process. Thus, the time scales for estimating $\gamma_{0}$ often depend on the experimental method used. Corrections are frequently necessary to account for any near-surface trace gas concentration gradients due to finite trace gas diffusion rates. Moreover, if the trace gas molecule of interest has a relatively low bulk or surface solubility, $\gamma_{0}$ may also have to be corrected for any re-vaporization due to finite solubility constraints. Only after both gas phase diffusion and solubility corrections have been made can the measured uptake coefficient be constrained to one or more of the gas/surface interfacial processes described above (Ammann and Pöschl, 2007; Pöschl et al., 2007, and references therein). In the case of unreactive gases, the resulting uptake coefficient can be identified with some combination of surface accommodation (adsorption) and bulk accommodation (absorption); see e.g. Kulmala and Wagner (2001) for formulae connecting bulk accommodation and uptake coefficients for condensation of a single component. For reactive molecules, the various types of reversible and irreversible surface reactions may proceed in series with surface adsorption and in parallel with absorption by the bulk phase. If either a surface or bulk phase reaction relaxes a Henry's law solubility constraint, controlling the rate of trace gas uptake, the symbols $\gamma_{\mathrm{reac}}, \gamma_{\mathrm{r}}, \gamma_{\mathrm{b}}, \gamma_{\mathrm{sol}}$ or even just $\gamma$ are often used to designate the uptake coefficient. If only surface reactions dominate the resulting uptake coefficient also may be designated as $\gamma_{\text {surf }}$ or $\gamma_{\mathrm{s}}$.

\subsection{Multiple uptake process models}

Given the potential complexity of the trace gas uptake process, phenomenological models are usually necessary both to evaluate the results of laboratory uptake experiments and to model the impacts of trace gas uptake on atmospheric processes. Phenomenological models currently used include:

1. Continuum flux model: Coupled differential rate equations of mass and heat transport using continuum flow formulations with correction factors for kinetic effects at gas-particle interface (Kulmala and Vesala, 1991; Vesala et al., 1997; Winkler et al., 2004).

2. Kinetic resistance model: Linear combination of flux resistances (decoupled and normalized fluxes) in analogy to resistances in electric circuits (Jayne et al., 1990; Hanson, 1997a; Ammann et al., 2003; Pöschl et al., 2007, Davidovits et al., 2006). 
3. Kinetic flux model: Coupled differential rate equations of mass transport and chemical reactions using gas kinetic formulations with correction factors for gas phase diffusion effects (Ammann and Pöschl, 2007; Pöschl et al., 2007).

\subsubsection{Key questions in the application of uptake process models}

The use of multiple phenomenological models and inconsistent terminology raises several key questions:

1. Are the different definitions, terminologies, parameters, and model formalisms fully consistent and equivalent? What are the exact relations between the different terms, parameters, and formalisms? Which of them are best suited for application in analysis of experimental trace gas uptake data, detailed small-scale atmospheric process models and in simplified large-scale atmospheric models?

2. What level of detail is required for efficient and consistent experimental and theoretical description and analysis of gas-particle interactions and their effects on atmosphere, climate, and human health (Ammann et al., 2007; Pöschl et al., 2007; Fuzzi et al., 2006)?

3. Is the solution of time-dependent equations describing mass transport and chemical reactions (coupled differential equations) fully consistent with the assumption of quasi-steady state (QSS) conditions and the use of simplified formalisms (resistance models)? If not, what are the relevant limitations for each level of description of gas-particle interactions in the atmosphere?

4. Is it necessary and possible to experimentally separate the processes and rate parameters of surface and bulk accommodation, $\alpha_{\mathrm{s}}$ and $\alpha_{b}$ (Pöschl et al., 2007)?

Some studies have explored and compared the applicability and consistency of different modeling approaches (e.g., Shiraiwa et al., 2009, 2010; Pfrang et al., 2010; and references therein). A general consensus on the approach and level of detail required or best suited for different types of applications, however, has not yet been reached. Thus, researchers should evaluate on a case-by-case basis, which model approach is best suited for their application. When surface saturation effects are negligible and the rates of surface reaction and desorption are much lower than the rate of surface-bulk transfer, then bulk and surface accommodation coefficients are near-identical and independent of gas phase composition. Under such conditions, it will normally not be necessary to resolve surface processes. For bulk condensation or evaporation and absorption of gases by liquid aerosol and cloud particles, it should normally be sufficient to determine and use a bulk accommodation coefficient (traditional "mass accommodation coefficient") to characterize the molecular kinetics of gas-to particle mass transfer. To describe non-linear effects of surface reaction or surface saturation in Langmuir-Hinshelwood-type reactions on solid or highly viscous liquid particles, however, it will be necessary to distinguish surface and bulk accommodation and to resolve molecular kinetics at the gas particle interface (sorption layer vs. quasi-static surface layer, etc.). In studies investigating individual species and processes under steadystate conditions, the traditional resistor model approach may be suitable. Under transient conditions and for multiple component/multi-process systems, however, it may be necessary to consider the full set of coupled differential equations describing mass transport and chemical reactions (Pöschl et al., 2007; and references therein).

\subsection{Thermodynamics of gas/liquid interfaces and trace gas uptake}

As noted above, interacting kinetic, thermodynamic and mass/heat transfer processes are involved in trace gas heterogeneous uptake. For instance, mass and heat transport occur simultaneously in condensation and evaporation processes due to the latent heat of phase transitions. Thus, simultaneous modeling of both mass and heat transport is often required for correct interpretation of experimental data, e.g. in experiments involving gas-to-particle conversion or high fluxes of gas accommodation to or evaporation from bulk liquid interfaces. Understanding the interplay of multiple physical and chemical processes is always challenging; however, convoluted interfacial processes pose a special challenge. Physicists and physical chemists have a long and successful history of characterizing thermodynamic, kinetic and heat/mass transfer processes in single phases and can draw on well established theories and models to describe and analyze their interplay in single phase systems. However, interfacial properties and processes are generally much less well characterized, often lacking even critical thermodynamic data.

\subsubsection{The gas/liquid interface}

At an air-aqueous interface, of a water droplet, for example, the strong hydrogen bonds associated with water give rise to a very high surface energy at the interface. The high surface energy may be reduced if surface-active species are present at the interface; this occurs spontaneously if the decrease in surface energy is sufficient to overcome the loss of full solvation by the surfactant species. Amphiphilic compounds, such as long-chain carboxylic acids and phospholipids, are good examples of this effect, since the hydrophilic head groups may be well solvated by surface water molecules, whereas the hydrophobic tails are not and are preferentially directed into the gas phase. However, even quite soluble compounds, such as dimethysulphoxide (DMSO) or ethanol, can be surface active (Donaldson, 1999; Mmereki et al., 2000; Donaldson 
and Vaida, 2006); that is, they may spontaneously partition to the air-water boundary in a proportion greater than that present in the bulk aqueous phase. Recently, some soluble inorganic anions (especially the larger halides) have also been shown to be surface active (Hu et al., 1995; Donaldson, 1999; Ghosal et al., 2005; Jungwirth and Tobias, 2006; Petersen and Saykally, 2006). The presence of compounds other than water at the interface clearly may affect both the surface properties and the chemistry there.

Insoluble, non-volatile surfactants, such as fatty acids, alcohols and other such species, have been identified in aerosols of marine origin (Tervahattu et al., 2002; Russell et al., 2010). Such insoluble compounds exist exclusively at the interface and their film properties are generally expressed in terms of a film equation of state, such as a 2-D van der Waals analog (Donaldson and Vaida, 2006). Such films may exist in 2-D analogs of gaseous, liquid and solid states, as the film becomes successively more compressed. By contrast, in studies of soluble surfactants, it is generally the surface excess, rather than the surface concentration, of adsorbate that is measured. This is defined as the amount of solute adsorbed to the surface relative to the amount of solvent, water in this case. The surface excess is given by the Gibbs equation (Adamson and Gast, 1997), which may be related to the adsorbed amount using an adsorption isotherm. For soluble surfactants, the concentration (or activity) dependence of the surface excess, at solute concentrations below phase separation, has generally been described by a Langmuir adsorption isotherm. This type of adsorption behavior implies that a finite number of independent adsorption sites exist at the aqueous surface, with a single enthalpy of adsorption, independent of surface coverage.

Analysis of adsorption isotherms obtained from the Gibbs equation (or from calibrated spectroscopic measurements), may yield the standard free energy for adsorption; the temperature dependence of this quantity then provides standard enthalpies and entropies. Chromatographic measurements also have been used to obtain 'surface partitioning coefficients' (Hartkopf and Karger, 1973; Hoff et al., 1993). Another approach (Donaldson and Anderson, 1999) considers the free energy for transferring one mole of species from either the gas or solution phase to the surface.

Several organic surface-active solutes have been treated this way over a range of temperatures, yielding values of $\Delta G^{0}, \Delta H^{0}$, and $\Delta \mathrm{S}^{0}$ for adsorption to the air-water interface. In general, there is reasonable agreement among the various methods (Hoff et al., 1993; Donaldson, 1999; Donaldson and Anderson, 1999; Mmereki et al., 2000; Roth et al., 2002) for values of adsorption enthalpies; values of the other thermochemical parameters are explicitly dependent on the choice of standard state. For compounds that are expected to be better solvated by water, the standard enthalpies of adsorption are different from those of vaporization, but are related to the infinite-dilution solvation enthalpies. These observations suggest that adsorption of gases to the water surface involves specific interactions, rather than the surface merely providing a site for condensation.

In addition to the challenges noted above, there are several areas were fundamental knowledge is simply not available. At this time, there are no quantitative experimental data describing thermochemical adsorption parameters for inorganic anions. The issues of specifying the surface standard state and determining activity coefficients for species adsorbed on water surfaces have been raised in only a handful of studies to date. The choice of standard state will influence the importance and magnitude of the activity coefficients, which quantify the departure from the "ideal" mixture, expressed in the dimensions of the standard state. In its most recent evaluation IUPAC (Crowley et al., 2010) recommends using a molar area of $A_{0}=3.74 \times 10^{7} \mathrm{~m}^{2} \mathrm{~mol}^{-1}$ as suggested by Kemball and Rideal (1946). Other choices (either explicit or implicit) include the "unit concentration" standard state, $1 \mathrm{~mol} \mathrm{~m}^{-2}$, the "unit surface pressure" standard state $\left(1 \mathrm{mN} \mathrm{m}^{-1}\right)$ and the "unit mole fraction" standard state, in which the surfactant surface coverage, $\theta,=1$. Regardless of which standard state is used, it is important to be aware that its choice will affect the values of the thermochemical parameters derived, so direct comparisons are not always possible between the various reports in the literature.

The presence of salts in aqueous solution may affect the ability of organic molecules to dissolve, through the saltingout effect. The salting-out effect refers to the decrease in aqueous solubility and increase in the activity coefficient of aqueous neutral nonpolar compounds by inorganic salts. Hardly any studies of this effect are present in the atmospheric literature. Demou and Donaldson (2002) reported that both hexanoic acid and 1-propanol display a reduction in their propensities to partition from the gas phase to the surface as salt concentration is increased. At the same time, the maximum surface excess of organic, $\Gamma_{i}^{\max }$, determined for salt solutions was larger than that for pure water and increased with increasing salt concentration.

\subsubsection{Trace gas uptake}

Trace gas exchange between the gas phase and solid particle or liquid droplet condensed phases are driven by thermodynamics, but are achieved by elementary kinetic and transport processes occurring at the phase interface and, sometimes, in the underlying bulk phase. Assuming that adsorption or desorption of a trace gas on a solid or liquid surface does not significantly affect the surface properties, the kinetic picture of adsorbing and desorbing molecules can be related to adsorption equilibrium; an assumption developed in the early surface science literature and also adopted by atmospheric chemists (Mozurkewich, 1993; Tabazadeh and Turco, 1993; Carslaw and Peter, 1997; Pöschl et al., 2001; Winkler et al., 2002; Ammann et al., 2003; Sumner et al., 2004; Bartels-Rausch et al., 2005). As noted above, these and many other studies have demonstrated that adsorption 
of atmospheric trace gases on relevant substrates can often be reasonably well described by simple Langmuir or BET isotherms. However, a thorough comprehensive treatment relating the kinetic derivation of equilibrium with the thermodynamic equilibrium is still lacking.

Under similar assumptions, i.e., when transfer of adsorbed molecules into the bulk does not affect bulk properties, the equilibrium between the gas and the bulk condensed phase, i.e., solubility, can be related to the exchange rate coefficients both between gas and surface and those between surface and bulk (Jayne et al., 1990; Hanson, 1997; Ammann and Pöschl, 2007; Pöschl et al., 2007; Remorov and George, 2006). While significant progress has been achieved in describing gas/aqueous phase equilibria (e.g. Clegg et al., 2001), the relation between the kinetic description and solution thermodynamics is even less established than for surface adsorption.

The rate at which the adsorbed interfacial molecules enter the bulk, i.e., bulk (mass) accommodation, has also been described as an activated process driven by the free energy change associated with solvation at the interface (Davidovits et al., 1995). On the other hand, the concentration of dissolved molecules at the interface at equilibrium can also be described by a Gibbs equation of state (e.g. Donaldson, 1999a), as described in the previous section. The resulting trace species surface concentration can become very significant for the partitioning of amphiphilic molecules between the gas and the condensed phase and for subsequent reactive loss processes (Djikaev and Tabazadeh, 2003; Ammann and Pöschl, 2007; Remorov and George, 2006).

Remorov and Bradwell (2005) have proposed a Langmuir model for the mass accommodation of non-reactive trace gas species on aqueous surfaces that differentiates between the effective interfacial solubility and bulk solubility. This difference leads to a model that predicts a time dependent bulk accommodation coefficient that decreases to a steady-state value as the interfacial region reaches Henry's law equilibrium with the gas phase trace gas concentration, allowing the model to reproduce both the near unity initial bulk accommodation values predicted by molecular dynamics simulations (as described in Section 4) and the smaller, longer time scale values obtained by many laboratory experiments. A Langmuir model based treatment is likely to be most pertinent to surface-active species and could be investigated experimentally by determining uptake coefficents' dependence on gaseous species concentration.

Links between kinetic descriptions of trace gas uptake and the thermodynamics of surface adsorption and bulk solubility are an active area of study for the atmospheric chemistry community. The development of a comprehensive framework will require considerable additional work and should certainly be a priority for future efforts.

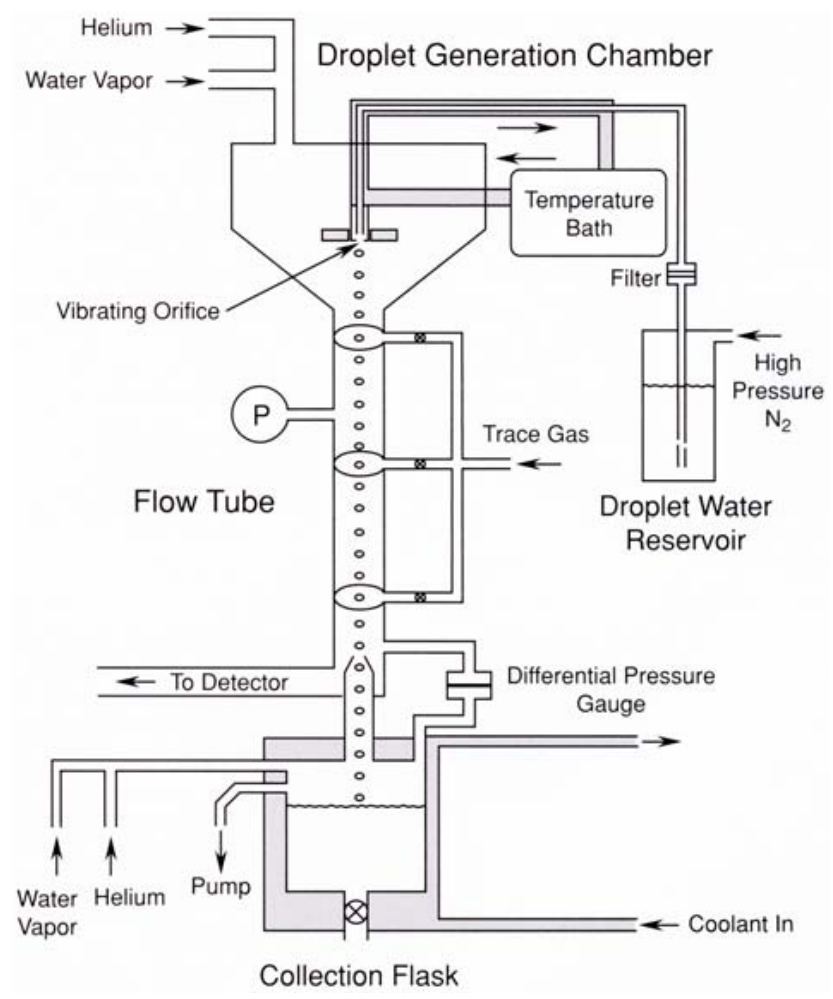

Fig. 2. Schematic diagram of typical droplet train flow reactor for measurement of uptake coefficients of trace gases into liquids (adapted from Jayne et al., 1992).

\section{Laboratory techniques for measurement of trace gas uptake}

A variety of techniques have been devised to meet the challenge of quantitative measurement of uptake kinetic parameters in multiphase systems. We present here only a brief description of four of the most successful methods that have been used in recent years for this purpose. Descriptions of these and additional techniques can be found in Danckwerts (1970), Kolb et al. (1995) and Davidovits et al. (2006).

\subsection{Droplet train flow reactor}

The droplet train flow reactor (Fig. 2) is a technique used to measure the rate of uptake of gases into liquid droplets for particle size $\sim 50-200 \mu \mathrm{m}$. It was first developed by the Aerodyne Research/Boston College Group (Worsnop et al., 1989) for measurement of accommodation coefficients of reactive trace gases in aqueous substrates.

It is comprised of a droplet generation chamber, which ejects droplets of uniform size and spacing via a vibrating orifice into a flow tube at linear flow velocities typically in the range (1500-4500) $\mathrm{cm} \mathrm{s}^{-1}$, and total pressure in the range 6-20 Torr. The surface/volume ratio in the reaction zone is typically $10^{-3} \mathrm{~cm}^{-1}$. Temperatures of the droplets lie in the range $\sim 260-290 \mathrm{~K}$ determined by the $\mathrm{H}_{2} \mathrm{O}$ vapor pressure in 


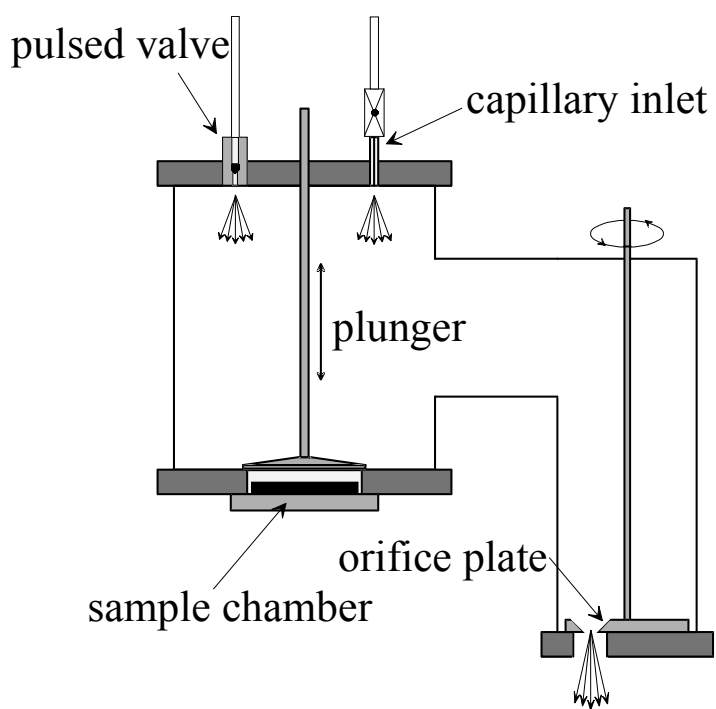

to the vacuum chamber and MS-detection

Fig. 3. Schematic of a Knudsen cell for the investigation of heterogeneous reactions using either continuous flow or pulsed gas admission. The rotatable orifice plate can put up to four molecular-beam forming orifices into line of sight with the ionizer of the mass spectrometric (MS) detector (from Caloz et al., 1997).

the tube. The reactant gas can enter the flow tube at various positions and changes in the concentration of the reactant gas, $\Delta_{n}$, are measured at the downstream end following exposure to the droplets. Typical detection techniques used for trace gases are mass spectroscopy and tunable diode laser infrared spectroscopy. The droplets leaving the interaction zone can also be collected and analyzed, typically using liquid chromatography methods, to determine changes in composition resulting from uptake.

The measured uptake coefficient can be evaluated from the ratio of the number of gas molecules taken up per second, $\left(F \Delta_{n}\right)$, over the total number of gas droplet collisions, $\left(N^{*} A N_{g} \bar{c}\right) / 4$ :

$\gamma=\frac{F \Delta_{n}}{N^{*} A N_{\mathrm{g}} \bar{c} / 4}$

where $F$ is the bath gas flow rate, $N^{*}$ the number of droplets which react with the gas, $A$ the droplet surface area, $N_{\mathrm{g}}$ the number of molecules of reactant gas per unit volume, and $\bar{c}$ the mean molecular speed. In practice it is convenient to measure the fractional change in gas concentration as a function of change in droplet surface area in contact with the trace gas. The surface area exposed to the trace gas is varied either by changing the applied frequency of the vibrating orifice or by a change in the droplet-gas interaction distance. The normal transit time in the reaction zone is $\sim 1-30 \mathrm{~ms}$, which is generally sufficient to avoid saturation of the surface. Very large uptake coefficients, requiring shorter contact times, can be achieved by injecting droplets transversely through holes in the side of a horizontal flow tube. If uptake coefficients are large, leading to significant concentration gradients, corrections may be required for gas phase diffusion. This requires knowledge of the diffusion coefficient of the trace gas in the appropriate carrier gas (usually He). Description of the mass transport in the gas phase is a key factor in obtaining accurate uptake rates using this technique.

An important aspect of the experimental technique is the careful control of all the conditions within the apparatus. All experimental parameters are computer monitored and controlled: pressure (typically 6-20 Torr); water partial pressure (1.7-16 Torr); temperature (260-290 K for pure water droplets); and gas interaction time $(1-30 \mathrm{~ms})$. The range of $\gamma$ values which can be measured is $10^{-3}-1.0$, although the detection limit is extended to $\sim 10^{-5}$ using liquid phase analysis. Experiments to date have been performed with water at various $\mathrm{pH}$ levels, aqueous salt solutions, organic liquids, and sulfuric acid from $20-70 \mathrm{wt} \% \mathrm{H}_{2} \mathrm{SO}_{4}$ (at temperatures down to $230 \mathrm{~K}$ ).

\subsection{Knudsen Cell}

The use of a Knudsen Cell for surface uptake studies was pioneered by David Golden and co-workers at Stanford Research Institute (SRI, now SRI International) (Golden et al., 1973; Quinlan et al., 1990). A schematic diagram of a typical Knudsen cell is shown in Fig. 3. Knudsen cells are operated at pressures $<10$ mTorr to ensure that the mean free path of the reactant gas exceeds the diameter of the exit orifice by at least a factor of three. In this way molecular flow conditions apply and gas phase diffusion effects are avoided. The reactant gas enters the cell at a known flow rate and exits it via an orifice of known size connected to a very low-pressure system. Detection is usually by modulated molecular beam mass spectrometry. As the reactant gas flows through the cell it is exposed to a sample surface, which may absorb, adsorb or react with the trace gas. Multiple orifices enable the measurement of the uptake rate as a function of pressure, which can be varied by up to a factor of two hundred. The first-order rate constant for the heterogeneous reaction, $k_{\mathrm{r}}$, and the uptake coefficient, $\gamma$, can be evaluated from the difference in reactant gas concentration both in the presence, $N_{\mathrm{r}}$, and absence, $N_{0}$, of the sample surface (which can be masked from the main cell volume) using the following equations:

$k_{\mathrm{r}}=k_{\mathrm{esc}}\left(\frac{N_{0}-N_{\mathrm{r}}}{N_{\mathrm{r}}}\right)$

$\gamma=\frac{A_{\mathrm{h}}}{A_{\mathrm{s}}}\left(\frac{N_{0}-N_{\mathrm{r}}}{N_{\mathrm{r}}}\right)$

Here $k_{\text {esc }}$ is the effective first-order rate constant for escape of the gas from the cell through the exit orifice, which can be evaluated experimentally or from molecular kinetic theory; $A_{\mathrm{h}}$ and $A_{\mathrm{s}}$ are the surface areas of the exit orifice and of 
the sample respectively. Typical surface/volume ratios are $4 \times 10^{-4}$ to $8 \times 10^{-2}$ and, by varying the relative size of $A_{\mathrm{h}}$ and $A_{\mathrm{s}}$ a range of $\gamma$ between 1 and $10^{-5}$, can be measured accurately. This range of $\gamma$ has been extended to $10^{-6}$ and below when the sealed-off sample compartment is used as a static reactor for the trapped gas phase at reaction times of minutes (Caloz et al., 1997).

The timescale for conducting uptake measurements in a Knudsen reactor is typically many seconds, often making surface saturation difficult to avoid and consideration must also be given to the possibility of re-evaporation into the gas phase following uptake, both producing time dependent uptake. Similarly, accumulation of products on the surface may change its reactivity. These effects lead to time dependent values of $\gamma$ that can provide useful information if measurements can adequately resolve the changes. Both pulsed and continuous gas flows have been used to investigate such behavior.

The main advantage of the Knudsen reactor is the avoidance of diffusion effects when uptake is very efficient. However, the need for very low operating pressures means that surfaces with high vapor pressures cannot be studied. This is a major limitation for atmospheric processes since it precludes studies on liquid water and ice at temperatures above $\sim 215 \mathrm{~K}$, although uptake on sulfuric acid at stratospheric temperatures can be studied. Uptake on solid substrates can be measured over a wide temperature range.

\subsection{Flow tube reactors}

\subsubsection{Coated wall flow tube reactor}

Coated and wetted wall flow tubes have been used extensively to measure uptake and reactions of gases with both liquid and solid surfaces. Figure 4 shows a schematic diagram of a typical flow tube system used in the investigation of the uptake of trace gases onto ice. The wall of the $1.5 \mathrm{~cm}$ diameter $\times \sim 50 \mathrm{~cm}$ length flow tube is coated with the condensed phase of interest (e.g. ice, salt/mineral dust layer or liquid film). The carrier gas (usually $\mathrm{He}, \mathrm{N}_{2}$ or Ar) travels under laminar plug flow at $500-3000 \mathrm{~cm} \mathrm{~s}^{-1}$ along the tube and enters the detector system downstream of the surface. The tube is jacketed allowing circulation of thermo-stated fluid to control temperature (range typically 190-350 K. Coated wall flow tube studies are generally carried out at low pressures (0.5-3 Torr) because of limitation in the diffusion rate of the reactant gas to the flow tube walls. For aqueous systems $\mathrm{H}_{2} \mathrm{O}$ is usually added to the carrier to prevent evaporative loss from the wall coating.

Reactant gas is added through a sliding injector. By varying the distance between the sliding injector and the detector, the time of exposure of the reactant gas to the condensed phase can be varied, allowing a pseudo-first order rate constant for removal of the gas, $k_{\mathrm{s}}$, to be measured, provided there is not significant surface saturation. Alternately the to-

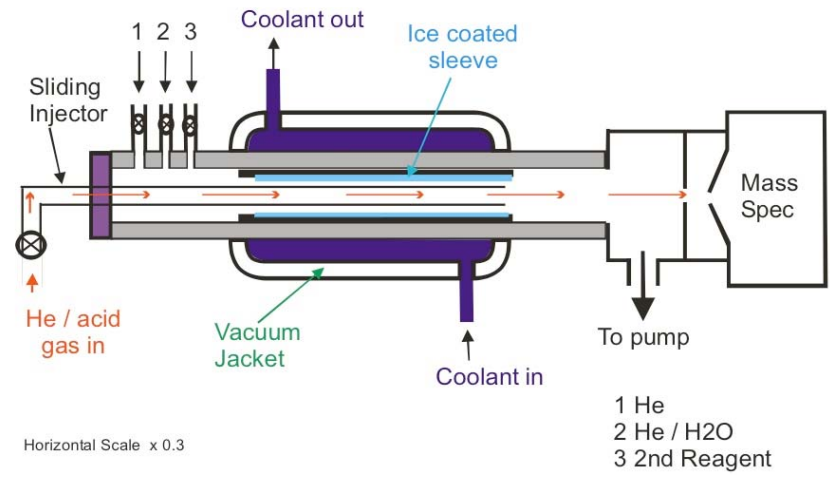

Fig. 4. Schematic of flow tube used for measurements of uptake of trace gases on ice (Symington, 2009). A smooth ice film is produced by freezing pure water on the inside of a liner inserted into the flow tube held at $258 \mathrm{~K}$. The trace gas diluted in $\mathrm{He}$ is introduced via a sliding injector.

tal amount of trace gas adsorbed on a prescribed surface area can be measured over a period of time at a fixed distance, providing information on surface coverage at saturation. This can be used to investigate adsorption characteristics and determine partitioning coefficients, e.g. to ice surfaces.

A variety of on-line sensitive detection schemes have been used, generally either spectroscopic or mass spectrometry with differential pumping (MS). Electron impact MS has been widely used. Additional sensitivity and selectivity has resulted from recent developments in chemical ionization mass spectrometry (CIMS). Optical detection techniques have included laser-induced fluorescence, UV and IR absorption and chemiluminescence.

The net uptake probability from coated wall flow tube experiments can be evaluated from:

$\gamma=\frac{2 r k_{\mathrm{s}}}{\bar{c}}$

where $r$ is the radius of the tube and $\bar{c}$ is the mean molecular speed. Correction of observed uptake rates for diffusion limitation can be made using the correction procedure described by Brown (1978), which can be applied if gas phase diffusion coefficients are known. Limits imposed by gas phase diffusion determines the upper limit for accurate uptake coefficient measurement of $\sim 0.2$ in wall coated flow tubes. Uptake coefficients down to $10^{-5}$ can be measured, making this a very versatile method for uptake measurements.

In the case of liquid surfaces a vertically mounted wetted wall flow tube is the favored configuration. The inside wall of the tube is completely covered with a slowly flowing film of the liquid of interest, which enters the tube via a lip or grooved joint to produce an even film. Thus the liquid surface is constantly renewed so that a surface of constant composition, free of saturation and reaction products, is obtained. An important limitation for aqueous liquids is the requirement for modest water vapor pressures to maintain a defined gas 


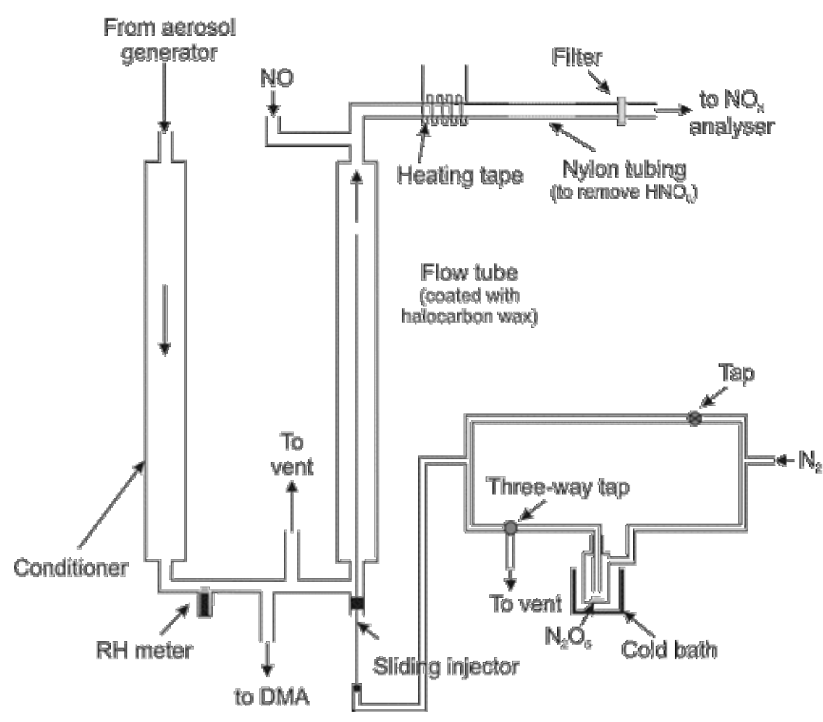

Fig. 5. Schematic of aerosol flow tube for measurements of uptake coefficients (from Badger et al., 2006). In this case $\mathrm{N}_{2} \mathrm{O}_{5}$ was measured using a chemiluminescence method after titration with NO added at the reactor exit.

diffusion regime. Thus, temperatures are restricted to 260 $293 \mathrm{~K}$. A second problem is to ensure that the liquid film is uniform and free of ripples, which can cause turbulence in the gas flow and augment diffusive transport to the surface.

In a recent paper Davis (2008) has published a detailed analysis of the interpretation of flow tube data for gas uptake measurements and reviewed the literature on flow tubes. The new analysis consolidates data obtained in earlier work for various flow rates and trace gas concentrations.

\subsubsection{Aerosol flow tube reactor}

The most realistic conditions for laboratory study of atmospheric heterogeneous reactions can be achieved with an entrained aerosol flow tube (AFT), in which the condensed phase takes the form of a poly-disperse sub-micron aerosol. The use of sub-micron particles or droplets overcomes the uptake rate-limitation caused by gas phase diffusion. This type of system was first used for uptake coefficient measurements of ammonia on sulfuric acid droplets by Robbins and Cadle (1953), Huntzicker et al. (1980) and McMurray et al. (1983), and the free radical, $\mathrm{HO}_{2}$, by Mozurkewich et al. (1987); it has subsequently been improved and developed by several groups, making use of novel technology in aerosol generation and characterization.

Figure 5 shows a schematic diagram for an aerosol flow system used to determine the uptake coefficient of $\mathrm{N}_{2} \mathrm{O}_{5}$ on aqueous aerosols. Submicron aerosols are generated in a nebuliser containing an aqueous solution or suspension of the desired substrate. The suspended particles are diluted in carrier gas (usually $\mathrm{N}_{2}$ or air), dried in a diffusion- dryer, re-humidified to the desired relative humidity, and passed through a conditioner where the aerosol equilibrates to the temperature and humidity conditions. The aerosol flow is split and one stream is directed to a differential mobility analyzer where the size distribution of the conditioned aerosol is determined. Charged particles need to be neutralized to avoid rapid loss if inlet lines and flow tube walls are not conductive (Guimbaud et al., 2002). Another stream enters the flow tube at the upstream end and the excess flow is vented. The total pressure is normally 1 bar and the flow speed $\sim 1-3 \mathrm{~cm} \mathrm{~s}^{-1}$, but the system can be operated at 100 mbar or less.

The reagent trace gas is diluted in carrier gas and enters the reactor through a sliding injector, allowing different exposure times to the aerosol, before detection at the reactor outlet. Attention needs to be given to the flow conditions required for establishment of laminar flow and mixing from the injector. This restricts measurements to the central portion of the flow tube but kinetic decays can normally be achieved over a useful time domain, and loss rate coefficients determined. Measurements for $k_{\mathrm{s}}$ have to be corrected for wall losses, hence uptake coefficients are determined by measuring the rate of loss of the reactant gas in the presence and absence of aerosol, with suitable corrections for concentration gradients produced by wall losses (Brown, 1978). The following equation is used to evaluate $\gamma$ :

$\gamma=\frac{4 k_{\mathrm{s}}}{\bar{c} S_{\mathrm{a}}}$

where $S_{\mathrm{a}}$ is the aerosol surface area per unit volume carrier gas. This is determined from the measured size distribution, which for soluble salt aerosols generated in a nebuliser, typically has a geometric standard deviation of $\sigma=1.5-2$ and a mean area weighted radius of $\sim 120 \mathrm{~nm}$. A calibrated scanning mobility particle sizer provides the integrated $S_{a}$, which can be varied over the range $10^{-5}-10^{-3} \mathrm{~cm}^{2} / \mathrm{cm}^{3}$. $S_{\text {a }}$ corresponds to the surface area of spherical particles with the same electrical mobility as those of interest. Therefore, a careful analysis is required to evaluate the true surface area for non-spherical particles. While the systematic error in $S_{\mathrm{a}}$ determined this way may be significant, e.g., $30 \%$ for mineral dust aerosol (Vlasenko et al., 2006), it is still much less than the large uncertainty associated with the evaluation of experiments using bulk powder substrates. This method has been used for accurate measurements of uptake coefficients of between 1.0 and $10^{-4}$ and on a range of inorganic and organic liquid particles and also on solid electrolytes and dusts. Careful control of all the conditions within the apparatus is achieved by using flow sensors and input flow valves operated under computer control.

Detection of trace gas concentration changes at the ppb level at 1 bar required for the AFT method has been mainly achieved via CIMS, which has potential for sensitive and selective measurement of a variety of species. Chemiluminescence analysis has been employed, e.g. for NOx species and for $\mathrm{HO}_{2}$. Alternately, the aerosol can be collected and the 


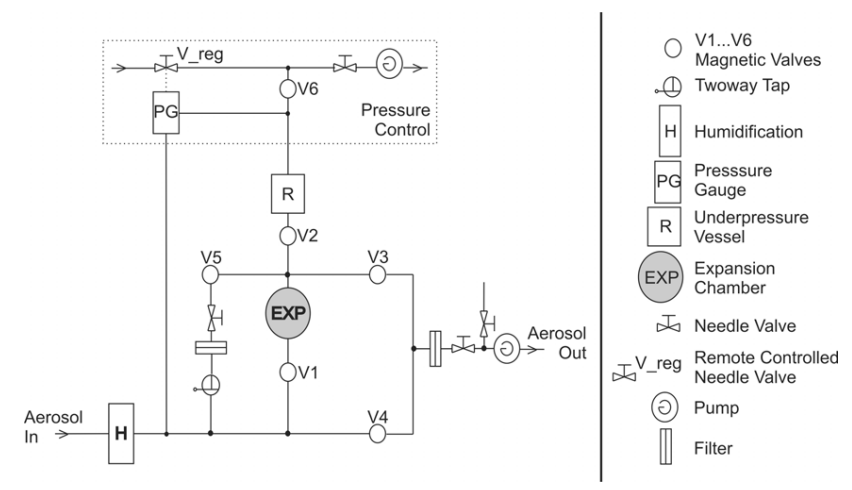

Fig. 6. Schematic diagram of the expansion cloud chamber showing the main components for pressure and flow control.

uptake determined by analysis of changes in the composition of the condensed phase.

\subsection{Cloud chamber}

Cloud chambers are commonly used for investigations of droplet formation and growth under well-defined thermophysical conditions. After vapour super-saturation has been established inside the measurement chamber, aerosol particles initiate formation of droplets, which subsequently grow by condensation. A schematic diagram of the expansion cloud chamber can be seen in Fig. 6. Vapor super-saturation is achieved by adiabatic expansion from the expansion chamber (EXP) allowing rapid establishment of well-defined and uniform conditions inside the measurement chamber, which has been previously charged with a well characterized monodisperse aerosol $\left(\sim 1.2 \times 10^{4}\right.$ particles $\left.\mathrm{cm}^{-3}\right)$ from an electrostatic classifier and a humidifier unit $(\mathrm{H})$. The pressure prior to expansion is measured on a gauge (PG) and the system attains thermal equilibrium with partial pressures in the expansion chamber slightly below those in the humidifier. Expansion is achieved by opening the valve (V2) between the expansion chamber and the much larger low-pressure buffer tank (R), leading to practically zero change in total pressure after the expansion.

Droplet growth is quantified using the constant angle Mie scattering (CAMS) method (Wagner, 1985), providing absolute, time-resolved, and non-invasive simultaneous determination of droplet diameter and number density. Mass and thermal accommodation coefficients can be determined from quantitative comparison of experimental and theoretical droplet growth curves. For the sizes and growth rates of the droplets considered a clear and accurate theoretical description is obtained using state-of-the-art condensation models (Vesala et al., 1997). Mass and heat fluxes to/from the droplets are calculated accounting for depletion of vapour as well as the production of latent heat in a population of growing droplets. As droplet growth is at least partly under gas kinetic control, both mass and heat flux need to be corrected by the corresponding transition regime correction factors (Fuchs and Sutugin, 1970)

$$
\beta_{\mathrm{m}}=\frac{1+\mathrm{Kn}}{1+\left(\frac{4}{3 \alpha_{\mathrm{m}}}+0.377\right) \mathrm{Kn}+\frac{4}{3 \alpha_{\mathrm{m}}} \mathrm{Kn}^{2}}
$$

and

$\beta_{\mathrm{t}}=\frac{1+\mathrm{Kn}_{\mathrm{t}}}{1+\left(\frac{4}{3 \alpha_{\mathrm{t}}}+0.377\right) \mathrm{Kn}_{\mathrm{t}}+\frac{4}{3 \alpha_{\mathrm{t}}} \mathrm{Kn}_{\mathrm{t}}^{2}}$,

respectively. Here, $\mathrm{Kn}$ and $\mathrm{Kn}_{\mathrm{t}}$ represent Knudsen numbers for vapour and carrier gas molecules given by the ratio of the corresponding mean effective free path and the droplet radius, and $\alpha_{\mathrm{m}}$ and $\alpha_{\mathrm{t}}$ denote mass and thermal accommodation coefficients. Variation of the total gas pressure inside the expansion chamber allows independent determination of $\alpha_{\mathrm{m}}$ and $\alpha_{\mathrm{t}}$, respectively (Winkler et al., 2004, 2006).

\section{Molecular dynamics simulations}

Some of the fundamental complexities of trace gas uptake by condensed phase systems have been outlined in Sect. 2, above. The question arises - can theoretical analyses and molecular simulation help? As available computer power has grown, molecular level simulation of complex chemical and physical processes has become an increasingly powerful tool for theoretical exploration. On the other hand, analytical expressions are also needed (e.g. Clement et al., 1996). In this section a summary of molecular simulation work on the trace gas uptake by aqueous systems is distilled from a recent review (Garrett et al., 2006).

Molecular simulations of aqueous interfaces provide detailed information about the structure, energetics, and dynamics of interface and molecular processes occurring at the boundary between vapour and liquid water (Pohorille and Benjamin, 1993; Pratt and Pohorille, 2002; Garrett et al., 2006). Many interaction potentials for water (Rahman, 1974; Lemberg and Stillinger, 1975; Rahman et al., 1975; Lie et al., 1976; Matsuoka et al., 1976; Stillinger and Rahman, 1978; Berendsen et al., 1981, 1987; Jorgensen et al., 1983; Carravetta and Clementi, 1984; Sprik and Klein, 1988; Niesar et al., 1989, 1990; Benjamin, 1991; Zhu et al., 1991; Caldwell and Kollman, 1995; Dang and Chang, 1997) have been used in simulations of aqueous interfaces (Townsend et al., 1985; Wilson et al., 1987a; Pohorille and Wilson, 1993; Taylor et al., 1996). The results from these simulations vary quantitatively from simulation to simulation, but they provide a consistent qualitative picture of the interface:

- The transition from vapour to liquid is over molecular length scales (e.g., $0.3-0.6 \mathrm{~nm}$ )

- The interface is rough on molecular length scales (e.g., $\sim 1 \mathrm{~nm}$ ) and fluctuates on time scales of $\sim 10^{-12} \mathrm{~s}$ 
- Molecular events at the interface (e.g., exchange of water molecules on the surface with those below the surface) occur on time scales of $10^{-12} \mathrm{~s}$

- The condensation and evaporation of water to and from the surface is equivalent to about 1 event per molecular area every $10^{-8} \mathrm{~s}$, and therefore do not play a significant role in the shorter time molecular events at the interface

- Calculated values of the surface tension of water for a variety of water models for many of the more commonly used potentials yield values that are within about $30 \%$ of experiment (Lee and Scott, 1980; Wilson et al., 1987b; Matsumoto and Kataoka, 1988; Zhu et al., 1991; Lie et al., 1993; Matsumoto et al., 1993; Alejandre et al., 1995; Dang and Chang, 1997; Taylor et al., 1996; Paul and Chandra, 2003; Vieceli et al., 2004).

Simulations of molecules interacting with the vapour/liquid interface of water have been performed for a variety of molecular species, (Pohorille and Benjamin, 1991; Matsumoto et al., 1993; Pohorille and Benjamin, 1993; Matsumoto, 1996; Sokhan and Tildesley, 1996; Tarek et al., 1996a, b; Taylor et al., 1997; Wilson and Pohorille, 1997; Benjamin, 1999; Taylor and Garrett, 1999; Dang and Feller, 2000; Shin and Abbott, 2001; Roeselova et al., 2003; Morita, 2003; Dang and Garrett, 2004; Paul and Chandra, 2004; Roeselova et al., 2004; Vacha et al., 2004; Vieceli et al., 2005; Canneaux et al., 2006; Minofar et al., 2007; Partay et al., 2007; Carignano et al., 2008; Mahiuddin et al., 2008; Morita and Garrett, 2008; Miller et al., 2009; Patel et al., 2009; Sun et al., 2009) including hydrophilic species such as ethanol, acids including oxalic and citric, amphiphilic molecules such as peptides, hydrophobic species such as oxygen and nitrogen, and radical species such as $\mathrm{OH}$ and $\mathrm{HO}_{2}$. The condensation of water on aqueous droplets is a special case of molecular uptake, but for molecular simulations, which treat all molecules as distinguishable, the methods used are the same as for other molecular species. Water adsorption has been studied extensively by molecular simulations (Nagayama and Tsuruta, 2003; Dang and Garrett, 2004; Morita et al., 2004a; Vacha et al., 2004; Vieceli et al., 2004). In an interesting recent study, a theoretical model of liquid water evaporation was presented based on transition state theory (TST) (Smith et al., 2006; Cappa et al., 2007).

Profiles of the variation in free energy have been computed for insertion of a molecule from the vapour, across the interface, and into the bulk liquid. The difference in free energy between the vapour and bulk liquid is equivalent to the free energy of solvation of the molecule, which can be related to the Henry's law coefficient. ${ }^{1}$ Similarly, the adsorption free

\footnotetext{
${ }^{1}$ The computed free energies use the thermodynamic definition of Ben-Naim and Marcus (1984), which gives the free energy of solvation in terms of the ratio of the number densities in the two
}

energy can be obtained from the free energy profiles and related to the surface excess. Comparison of computed (Pohorille and Benjamin, 1991; Pohorille and Wilson, 1993; Taylor et al., 1997; Wilson and Pohorille, 1997; Benjamin, 1999; Taylor and Garrett, 1999; Dang and Feller, 2000; Shin and Abbott, 2001; Dang and Garrett, 2004; Paul and Chandra, 2004; Roeselova et al., 2004; Vacha et al., 2004; Vieceli et al., 2005; Canneaux et al., 2006; Partay et al., 2007; Carignano et al., 2008; Morita and Garrett, 2008; Patel et al., 2009; Sun et al., 2009) and experimental (Ben-Naim and Marcus, 1984; Castro et al., 1991; Hanson et al., 1992; Dabkowski et al., 1996; Karpovich and Ray, 1998; Allen et al., 1999; Sander, 1999; Autrey et al., 2004) solvation and adsorption free energies provide a test of the accuracy of the molecular interactions used in simulations of uptake. The comparisons show that the interaction potentials are capable of reproducing energetic quantities to within several $\mathrm{kJ} / \mathrm{mol}$. The free energy profiles indicate that small hydrophilic molecules are surface active, that is, their free energy at the interface is lower than in the bulk. Furthermore, the free energy profiles exhibit only small intrinsic barriers (typically less than $5 \mathrm{~kJ} / \mathrm{mol}$ ) for moving the solute from the bulk liquid to the interface, and these barriers are much smaller than those for desorption of the molecule into the vapour. These results disagree with the interpretation of uptake experiments (Davidovits et al., 1995, 2005) and the disagreement provided motivation to calculate kinetic and dynamical quantities to explain the discrepancy.

Ethanol uptake is a prototypical example that has been studied in detail by a few research groups (Wilson and Pohorille, 1997; Taylor et al., 1997; Taylor and Garrett, 1999; Taylor and Shields, 2003; Canneaux et al., 2006). Transition state theory using the free energy profile (Chandler, 1978; Hynes, 1985; Schenter et al., 2003) was employed to calculate absorption and desorption rate constants for the prototypical example of ethanol uptake (Taylor and Garrett, 1999). Grote-Hynes theory (Grote and Hynes, 1980) has been used in two cases, (Taylor and Garrett, 1999; Shin and Abbott, 2001) including for ethanol uptake, to correct for effects of solvent dynamics on the reaction rates. The calculated absorption rate for ethanol is $10^{10} \mathrm{~s}^{-1}$, which is about a factor of 1000 times faster than the desorption rate of $10^{7} \mathrm{~s}^{-1}$ (Taylor and Garrett, 1999). The time scale for absorption from the interface to the bulk liquid is comparable with the time scale for diffusing about $1 \mathrm{~nm}$ into the liquid, which is the length scale at which the free energy profile goes to the bulk liquid value. Dynamical simulations, or classical trajectories, have been used to test whether there are dynamical bottlenecks that do not appear in the energetic (free energy) calculations. If the interpretation of uptake experiments is correct, the time scale for molecules to move from the interface to the bulk liquid should be comparable or longer than the desorption time

phases. The experimental values are for the low concentration limit, which is the same condition as the computed values. 
scale of $10^{-7} \mathrm{~s}$, while the kinetic and diffusion lifetimes computed for absorption are about 3 orders of magnitude faster, $\sim 10^{-10}$ s. Wilson and Pohorille (1997) calculated a $30 \mathrm{~ns}$ trajectory with ethanol initially at the liquid/vapour interface. The trajectory was observed to move between the surface and bulk a number of times indicating a time scale on the order of a $10^{-9} \mathrm{~s}$. Similar results were obtained for simulations of DMSO on water (Benjamin, 1999). Therefore, the calculated time scale for molecules to move from the interface to the bulk liquid is fast, in disagreement with interpretations of experimental measurements (Davidovits et al., 1995, 2006).

Calculations have also been performed to study the initial sticking, or surface accommodation, of the collisions of several molecules with water surfaces (Wilson and Pohorille, 1997; Nagayama and Tsuruta, 2003; Morita, 2003; Roeselova et al., 2004; Tsuruta and Nagayama, 2004; Vieceli et al., 2004; Morita et al., 2004a, b; Vieceli et al., 2005). The conclusion from these studies is that for collisions of hydrophilic molecules near room temperature nearly all molecules initially stick to the surface. For the one hydrophobic molecule studied (ozone), the predicted mass accommodation coefficient was 0.047 and the thermal accommodation coefficient was 0.90 . The picture that emerges from these studies is that liquid water is very efficient at dissipating the translational energy of the incident molecule, leading to rapid equilibration of the majority of the incident molecules. After a relatively short period of time (on the order of $10^{-9}$ to $10^{-8} \mathrm{~s}$ ) the probability of desorbing and absorbing are determined by the kinetics of the evaporation and absorption processes.

The major conclusion from all of these studies is that both scattering and kinetic calculations yield values for the mass accommodation coefficient close to unity except for molecules with small solubility in water (e.g., ozone). This conclusion includes water condensation, where values for mass accommodation coefficient are calculated to be unity (Vieceli et al., 2004; Nagayama et al., 2003; Dang and Garrett, 2004; Morita et al., 2004a; Vacha et al., 2004; Vieceli et al., 2005). The more recent study of water evaporation using a TST model (Cappa et al., 2007) gave results that were fitted to agree with their experimental observations that yielded an evaporation coefficient of about 0.6 (Smith et al., 2006; Drisdell et al., 2008). The discrepancy between the experimental and computational interpretations of mass accommodation requires further investigations to understand the limits of both the simulation and experimental approaches.

The simulation methods are by necessity approximate. The size and length of water simulations are generally limited to hundreds to thousands of molecules for time scales of $10^{-8} \mathrm{~s}$ or less. Periodic boundary conditions Allen and Tildesley, 1987) are used to extend the effective size of the simulations and mitigate the effects of small simulation sizes. The size of the system is also connected with how the long-range interactions are treated. Many early simulations truncated all interaction potentials past some cutoff value (typically on the order of $1 \mathrm{~nm}$ ), while more recent simulations typically use Ewald summation methods (Ewald, 1921) to treat the long-range electrostatic interactions more accurately. Computed values must be converged with respect to increasing the size of system and the length of the simulations. There is limited evidence that simulation sizes on the order of 1000 water molecules are sufficient when used with Ewald summation methods. A recent study has shown that lengths of simulations of $10^{-9} \mathrm{~s}$ or longer are needed to converge properties such as the surface tension and that shorter time scales can lead to errors on the order of about $30 \%$ (Ismail et al., 2006). Another consideration is that most simulations of molecular uptake do not account for finite concentration effects. In the uptake experiments by (Jayne et al., 1991) the gas phase density of ethanol was about $5 \times 10^{13}$ molecules $\mathrm{cm}^{-3}$, so that at saturation the aqueous phase concentration is on the order of $2 \times 10^{17}$ molecules $\mathrm{cm}^{-3}$ (about $4 \times 10^{-4} \mathrm{M}$ or a mole fraction of $7 \times 10^{-6}$ ) and surface concentration is about $1 \times 10^{18}$ molecules cm $\mathrm{cm}^{-3}$ (about $2 \times 10^{-3}$ $\mathrm{M}$ or a mole fraction of $4 \times 10^{-5}$ ). Stewart et al. (2003) performed simulations of the free energy profile for ethanol uptake into a 0.059 mole fraction aqueous ethanol solution and found the same qualitative behavior as in the infinitely dilute case. Changes of solvation and adsorption free energies with concentration were less than the uncertainties in the computed values. In this case of finite concentration the free energy profile rose monotonically from the surface into the bulk liquid and did not display an intrinsic barrier. Additional work to clarify the role of concentration effects on free energy profiles would be helpful.

\section{Recommendations for further work}

Future work to understand trace gas uptake using molecular simulation methods will benefit greatly from advances in computing hardware and software. Almost all previous simulations of molecular processes at the air/liquid interface have relied on approximate analytical expressions for interaction potentials, which are fitted to experimental data. Advanced computing resources now enable reliable simulations of liquid interfaces using electronic structure methods to describe the molecular interactions (Kuo and Mundy, 2004; Mundy et al., 2008). Studies that use more reliable interaction potentials such as electronic structure methods will help to understand the influence of the interaction potential on the structure, energetics, and dynamics at liquid interfaces, and address criticisms (Davidovits et al., 2005; Worsnop et al., 2004a, b) of previous simulation results. More work is also needed to bridge the gap between the molecular scales treated in simulations to the macroscropic scale of the experiments. Uptake of molecules by water droplets is a macroscopic process that is controlled by mass transport in the gas and liquid phases, and can be influenced by molecular-scale processes occurring at the vapor/liquid interface. Multi-scale 
models that accurately include molecular scale phenomena in continuum models are required to provide a clear understanding of molecular processes in the uptake phenomenon.

\section{Overview of current experimental results}

In this section we review what we have determined experimentally and outline what we need to learn. A discussion of the characteristics of each surface type, the general behaviour of uptake kinetics on each surface, and the outstanding challenges is presented below.

\subsection{Results and challenges for aqueous liquid surfaces}

The uptake of trace gaseous species by aqueous liquid surfaces has most recently been reviewed by Davidovits et al. (2006). As noted above, liquid aqueous surfaces of most relevance to atmospheric PM include nearly pure water (cloud and fog droplets), inorganic acid/water solutions (stratospheric and newly nucleated tropospheric aerosol droplets), halide salt solutions (sea salt aerosols), ammonia neutralized acid/water solutions (secondary inorganic aerosol droplets), and all of the above with dissolved soluble organics, organic surfactant coatings, or mixed aqueous organic liquid phases (primary and secondary aerosol droplets with organic components). The influence of organic films on trace gas uptake by aqueous surfaces was the focus of a recent review by (Donaldson and Vaida, 2006).

The experimental community has developed a number of techniques for measuring trace gas uptake on liquid surfaces, including wetted wall, droplet train, bubble train, and aerosol flow reactors, Knudsen cell reactors, coaxial liquid jets, single suspended droplet/laser probe methods, impinging gas/liquid flows, ambient pressure aerosol chambers, and liquid surface/molecular beam scattering chambers. Brief descriptions of several these techniques have been presented in section 3 and further examples can be found in Kolb et al. (1995) and Davidovits et al. (2006).

The general status of our knowledge of trace gas uptake on key classes of aqueous liquid surfaces and current research challenges are reviewed below.

\subsubsection{Laboratory measurement of uptake of water molecules onto water surfaces}

Numerous experimental methods have been proposed during the past few decades to determine mass and thermal accommodation coefficients (see, e.g., Davis, 2006 and Davidovits et al., 2006). Recent focus has been on experiments employing droplet trains or liquid jets (Li et al., 2001; Cappa et al., 2005; Smith et al., 2006), expansion cloud chambers (Winkler et al., 2004, 2006), laminar diffusion flow reactors (Voigtländer et al., 2007) and electrodynamic levitation (Shaw and Lamb, 1999; Zientara et al., 2008).
The outcomes of these experiments, however, show considerable discrepancies (Davidovits et al., 2004). Droplet train experiments ( $\mathrm{Li}$ et al., 2001) yield mass accommodation coefficients of the order of 0.2 , with a significant negative temperature dependence (the accommodation coefficient decreases with increasing temperature). On the other hand, expansion cloud chamber experiments require and allow determination of both thermal and mass accommodation coefficients. Both coefficients are found to be close to 1 within experimental error (Winkler et al., 2004, 2006). No temperature dependence was observed. The most recent electrodynamic levitation experiments yield temperature dependent results in excellent agreement with the droplet train measurements (Zientara et al., 2008). Recent experiments with liquid jet and droplet trains in very low pressure chambers measure evaporation coefficient values near 0.6 (Smith et al., 2006; Drisdell et al., 2008). (Evaporation coefficients are believed to be equivalent to mass accommodation coefficients via microscopic reversibility.)

As a result of different values reported based on different experimental techniques a review of data interpretation is needed. In the interpretation of droplet train data the resistance model is usually used which includes the mass accommodation coefficient. To correct for gas phase diffusion the Fuchs-Sutugin equation is applied (Worsnop et al., 2001). This requires knowledge of the Knudsen number (mean free path/droplet radius) that varies with pressure and droplet size.

In expansion cloud chamber experiments (Winkler et al., 2004, 2006) droplet growth curves were measured by Constant-Angle Mie Scattering (Wagner, 1985) for water vapor saturation ratios ranging from 1.02 to 1.5 . Accommodation coefficients were obtained from comparison of measured growth rates to quasi-steady state droplet growth calculations (Kulmala and Vesala, 1991; Kulmala et al., 1993a; Vesala et al., 1997) to account for the convective transport in the vicinity of the growing droplets (Stefan flow). The characteristic times for the concentration and temperature profiles to relax to steady state can be determined by standard expressions (see, e.g. Seinfeld and Pandis, 1998). For the experimental conditions of Winkler et al. $(2004,2006)$ these times are typically in the range from 0.1 to $1 \%$ of the growth times, and never exceed $3.2 \%$. Accordingly, nonsteady state effects can be disregarded in the Winkler et al. $(2004,2006)$ experiments. All but one experiment reported by Winkler et al. $(2004,2006)$ occurred for supersaturations of $\sim 0.2$ to 0.5 ., more data close to atmospheric conditions (supersaturations $\leq 0.02$ ) would be desirable. Voigtländer et al. (2007) analyzed droplet growth in a laminar diffusion flow reactor using droplet growth models similar to those of Winkler et al. and obtained similar results, estimating $0.3<\alpha<1.0$ at 275-277 K.

Single water droplet evaporation experiments have been reported by Zientara et al. (2008), in a series of papers culminating in a thorough temperature dependant study. Because they carried out the evaporation experiments at atmospheric 
pressure, the reported results must be derived using a continuum theory modified by incorporating an effective gas phase diffusivity and an effective thermal conductivity, which is similar to, but they suggest is more complete, than the model used by Winkler et al. $(2004,2006)$ to analyze their expansion cloud chamber growth data. The Zientara et al. (2008) temperature dependent evaporation coefficient results agree very well in both magnitude and temperature trend with the droplet train mass accommodation coefficient results reported by $\mathrm{Li}$ et al. (2001). It should be noted however, that the relative humidity in the chamber applied by Zientara et al. (2008) is not directly measured but obtained from a 4-parameter fit (the other parameters are the accommodation coefficients and droplet charge). This is may introduce uncertainty in the data, since net uptake, and thus, evaporation processes and calculated accommodation coefficients are very sensitive to relative humidity.

In addition, novel studies of water jet and water droplet train evaporation under low pressure, nearly free evaporation conditions have been reported by the Saykally/Cohen groups at UC Berkeley (Cappa et al., 2005; Smith et al., 2006; Drisdell et al., 2008). Both of these experiments concluded that the evaporation coefficient was significantly less than 1 , indicating a rate limiting activation barrier for evaporation (and invoking microscopic reversibility, for mass accommodation). The second, more definitive Smith et al. (2006) experiment reported a weak temperature dependence between 245 and $298 \mathrm{~K}$ with an average $\alpha$ value of $0.62 \pm 0.09$. The method used by Smith et al. (2006) has been extended to $\mathrm{D}_{2} \mathrm{O}$ by Disdell et al. (2008) yielding an evaporation/mass accommodation coefficient of $0.57 \pm 0.06$ for roughly the same temperature range. Whether the air/water interface structural and kinetic properties for the low gas pressure, nearly free evaporation conditions of the Berkeley experiments and the atmospheric pressure, near equilibrium conditions of the Zientara et al. (2008) experiments are comparable remains an open question.

\section{Recommendations for further work}

See combined recommendations for water vapour and trace gas uptake at the end of Sect. 5.1.7.

\subsubsection{Field determination of uptake of water onto water surfaces}

Determination of mass accommodation coefficient from socalled "droplet closure" measurement in the field has been attempted by Fountoukis et al. (2007). An "optimum" value of $\alpha \sim 0.06$ was suggested as being best able to reproduce the observed cloud droplet number concentration in a range of airborne and ground-based cloud experiments. This was deduced from simulations using the cloud parcel model of (Fountoukis and Nenes, 2005) constrained by typical up draft velocities. The model adopts the conventional simultaneous solution of the mass and heat flux to the surfaces of a population of growing droplets and, whilst it is undefined whether the derived $\alpha$ value was equivalent to $\alpha_{m} / \alpha_{\mathrm{s}}$ or $\alpha_{b}$, it would appear that the formulation of the model is sufficiently similar to that used in the Winkler et al. $(2004,2006)$ to derive $\alpha_{m} / \alpha_{\mathrm{s}}$ values of unity from expansion chamber studies, to infer that the derived value is $\alpha_{\mathrm{m}} / \alpha_{\mathrm{s}} \sim 1$. In the Fountoukis and Nenes (2005) study, it appears that any value greater than 0.06 is able to reproduce the measured droplet number within the measurement error. Thus it appears appropriate to claim that $\alpha_{m} / \alpha_{\mathrm{s}} \sim 0.06$ is a lower limit to the value derived from the closure study, rather than the optimum value. It is also difficult to assess whether the error introduced into the predicted droplet number concentration through realistic quantification of the error in the input up draft velocity is included in the reported error bars. Other studies have achieved closure with a range of chosen accommodation coefficient values from 0.04 to 1.00 (see Table 7 in McFiggans et al., 2006, and references therein) and values as low as $10^{-4}$ (Cantrell et al., 2001) and $10^{-5}$ (Chuang, 2003) have even been inferred for specific periods in atmospheric studies.

Laaksonen et al. (2005) advocate that, since they analyze their expansion chamber water droplet growth data with the same model equations generally used to predict cloud droplet growth, to be consistent with their laboratory data (Winkler et al., 2004, 2006) a mass accommodation coefficient of unity should be used. However, Zientara et al. (2008) have recently questioned whether the heat and mass flux model used for their experimental droplet growth data analysis by Winkler et al. $(2004,2006)$ is complete. The importance of proper mass and thermal accommodation coefficient values for cloud microphysical studies was emphasized previously by Kulmala et al. (1993b).

Owing to the significant error in both modelled (through input updraft velocities) and measured droplet number, it is difficult to reliably derive values for accommodation coefficient from cloud droplet closure studies. Moreover, since there are invariably trace components in cloud droplets (the original seed aerosol components), and indeed suppression of mass transfer rate by these components have been invoked as a reason for the choice of low accommodation coefficients, such closure studies could not be reliably used to derive accommodation coefficients of water on pure water even in the absence of measurement or modelling error. The use of such closure studies is not, however, greatly diminished by the above statements since the broad inference of suppression of the "effective" accommodation coefficient may be used as evidence for some suppression of the uptake kinetics (albeit not strictly the mass accommodation coefficient).

While comprehensive quantification of the impact of the water vapour accommodation coefficient on cloud properties is beyond the scope of this review, it will likely be significant and non-linear. In particular, it should be noted that a recent cloud parcel model study of aerosol PM and 
updraft limitations on cloud droplet formation (with an implicit assumption of unity accommodation coefficient) identified a number regimes that may exhibit significant sensitivity to the use of such an appropriate "effective" accommodation coefficient (Reutter et al., 2009).

There have also been several attempts to investigate potential "suppression of growth kinetics" in smog chambers (Asa-Awuku et al., 2010; Engelhart et al., 2008) and in field studies (Bougiatioti et al., 2009) by adoption of Köhler theory analysis (Padró et al., 2007). It is unclear whether such analyses are reliable, as discussed in the anonymous review of Asa-Awuku et al. (2010), since there are several alternative potential reasons for discrepancy between the size of activated droplets from sample and from inorganic calibration aerosol. In any case, such studies should yield a measure of suppression relative to that of the kinetics of growth by accommodation of water on calibration salt solution droplets (e.g. Shantz et al., 2010).

It is widely known and accepted that a coupled consideration of heat and mass flux must be used to realistically treat growth of cloud droplets in a depleting water vapour field, such as those often encountered in the atmosphere. Since growth is largely in the continuum regime, the appropriate growth laws are well defined and use of a flux matching correction such as the widely used Fuchs-Sutugin (1970) correction or some other such treatment of transition regime mass flux can be used to represent deviation from continuum regime growth. It has been contended that in order to consistently treat growth of cloud droplets in competition for the available water, that an accommodation coefficient of unity is required and any discrepancy in droplet closure experiments must therefore be attributed to some other suppression of growth kinetics not incorporated in the accommodation coefficient (Laaksonen et al., 2005).

It is unknown whether apparent discrepancies in droplet closure exercises (see above) result from the presence of film forming trace organic components, which have been hypothesized to be able to inhibit water uptake or from some other real suppression of uptake kinetics. An alternative possibility is that the apparent discrepancy results from uncertainties in the closure measurements. Since the source (and indeed existence) of systematic discrepancy is in dispute, the level of detail that must be included in atmospheric model treatments of droplet growth is currently unclear. There are a large number of potential candidates for film-forming compounds and their properties vary widely. Whether a modified "effective" mass accommodation coefficient tuned to best represent available atmospheric measurements may reasonably be used to capture inhibition of uptake kinetics is unknown. Pöschl et al. (2007) have provided a framework to describe the kinetics of transport across the gas / liquid interface in which the formal description of uptake kinetic limitation clearly indicates that accommodation coefficient modification may not be the most informative or meaningful correction.

\section{Recommendations for further work}

See combined recommendations for water vapour and trace gas uptake at the end of Sect. 5.1.7.

\subsubsection{Trace gas uptake onto water surfaces}

Measured mass accommodation coefficients using a range of experimental techniques for a variety of trace species are generally between 0.01 and 1.0 (Davidovits et al., 2006). Some temperature dependent studies show mass accommodation coefficients tend to increase with decreasing temperature, with experimentally accessible surface temperatures ranging from $\sim 300$ to $265 \mathrm{~K}$, although not all experimental techniques can access the super-cooled liquid water temperatures below $272 \mathrm{~K}$ (Davidovits et al., 2006). However, there is still discrepancy between different techniques in the temperature-dependence of the reported mass accommodation coefficients: data sets obtained applying expansion cloud chambers typically report no temperature dependence for the mass and thermal accommodation coefficients (e.g. Rudolf et al., 2001; Winkler et al., 2004).

For reasons discussed above, the determination of mass accommodation coefficients for relatively insoluble species is much more difficult since bulk Henry's law constraints may cause evaporative losses before quantifiable amounts of trace gases are absorbed. In addition, surface-active molecules with relatively low solubility may adsorb to the surface rather than absorb into the bulk. In this case, it becomes difficult to experimentally distinguish between surface and bulk accommodation.

One common practice to relieve bulk accommodation Henry's law solubility constraints has been to reactively scavenge accommodated molecules by adding acid $\left(\mathrm{H}^{+}\right)$or reactive anions $\left(\mathrm{Br}^{-}, \mathrm{I}^{-}\right.$, etc.). However, subsequent studies have shown that hydronium ions, heavy halide ions, and some other common ions are actually surface active, with interfacial mole fractions exceeding bulk mixing ratios (Jungwirth and Tobias, 2006). Since surface reactions proceed in parallel with mass accommodation (Hanson, 1997a, 1998), the resulting surface reactions, like surface adsorption, are also difficult to distinguish from bulk accommodation. Recent studies have even indicated that hydronium ions have a significant surface excess even in pure water (Buch et al., 2007), meaning that acid catalyzed reactions, like D/H isotopic exchange, may occur much more rapidly on water surfaces that previously expected. Further work is required to allow clear identification of $\gamma$ with $\alpha$. (For example, see the discussion below about ozone uptake, in Sect. 5.1.5).

There are studies, however, that may distinguish between the uptake and mass accommodation coefficients for trace gases, assuming that co-deposition of a trace species along with larger depositional flux of water vapour is equivalent to trace gas accommodation on a water surface near equilibrium with its vapour. For instance, Rudolf et al. (2001) report mass 
accommodation coefficients of unity for nitric acid on nitric acid/water surfaces, based on cloud chamber experiments similar to those reported by Winkler et al. (2004). Boy et al. (2005) report a successful closure between the production rates, sinks and observed concentrations of $\mathrm{H}_{2} \mathrm{SO}_{4}$ assuming a mass accommodation coefficient of unity for sulphuric acid onto ambient aerosol particles. Evaporation rates of dicarboxylic acids from aqueous solution surfaces also seem to be consistent with reported saturation vapour pressures if mass accommodation coefficients close to unity (or at least not smaller than 0.1) are used for both the acids and water (Riipinen et al., 2007).

When considering reactive uptake on pure water, reactions are generally restricted to species that readily hydrolyze (e.g. $\mathrm{N}_{2} \mathrm{O}_{5}, \mathrm{HCHO}, \mathrm{CH}_{3} \mathrm{CHO}$ ) or involve isotope exchange (e.g. $\mathrm{C}_{2} \mathrm{D}_{5} \mathrm{OD}, \mathrm{CD}_{3} \mathrm{COOD}, \mathrm{D}_{2} \mathrm{O}$ ) (Shi et al., 1999; $\mathrm{Li}$ et al., 2001). Irreversible uptake depends on the occurrence of rapid reaction/isotopic exchange in the interface region, which depends on the local availability of water molecules. Under such circumstances irreversible uptake to water droplets may be controlled by mass accommodation, but may also be affected by surface reactivity. Whether hydrolysis or isotopic exchange reactions occur within the interface region, in the bulk liquid phase (after mass accommodation), or both can be difficult to determine (Clifford et al., 2007; Audura and Donaldson, 2009). Methods that measure reactive uptake with very high time resolution or as a function of droplet surface/volume ratio may be able to determine the relative importance of surface versus bulk phase reactions (Hanson and Lovejoy, 1996; Hanson, 1998).

\section{Recommendations for further work}

See combined recommendations for water vapour and trace gas uptake at the end of Sect. 5.1.7

\subsubsection{Stable trace gas uptake on acidic aqueous surfaces}

Because of the importance of heterogeneous processes on binary water/sulphuric acid and ternary water/nitric acid/sulphuric acid PM in stratospheric ozone depletion a large number of studies have investigated uptake processes on these acid solutions (see Davidovits et al., 2006 for additional discussions and references). Weaker acid solutions, meant to approximate aqueous tropospheric particulate matter have also been investigated for some trace gases. Important reactive uptake processes involving $\mathrm{N}_{2} \mathrm{O}_{5}, \mathrm{ClONO}_{2}$, $\mathrm{BrONO}_{2}$, and the permutations of $\mathrm{HX}+\mathrm{HOX}(\mathrm{X}=\mathrm{Cl}, \mathrm{Br})$ have been studied by multiple experimental techniques producing results with generally reasonable agreement that have been incorporated into stratospheric chemistry models.

Bulk accommodation uptake studies of $\mathrm{HCl}, \mathrm{HCHO}$ and other moderately soluble trace gases have also been studied by multiple techniques as a function of both tempera- ture and acid concentration. $\mathrm{HCl}$ uptake as a function of sulphuric acid weight per cent performed by aerosol flow reactor and droplet train flow reactor techniques disagree for relatively low acid concentrations at temperatures around $270 \mathrm{~K}$. Aerosol flow reactor results for $26 \mathrm{wt} . \%$ sulphuric acid using $\mathrm{HOCl}$ to scavenge $\mathrm{HCl}$ to avoid Henry's law solubility constraints yielded a significantly higher uptake coefficient than uptake on pure water and weak sulphuric acid solutions using the droplet train flow reactor. Similar discrepancies occur for droplet train versus aerosol flow reactor results measuring uptake of $\mathrm{NH}_{3}$ on pure water and dilute sulphuric acid solutions (Worsnop et al., 2004a; Hanson and Kosciuch, 2004); both techniques do measure an uptake coefficient of $\sim 1$ above $\sim 50 \mathrm{wt} . \%$ acid. Finally, uptake coefficients of methane sulphonic acid on dilute sulphuric acid obtained using the aerosol flow reactor were much higher than those obtained on pure water at similar temperatures using droplet train flow reactors in two separate laboratories. These differences may be due to inaccurate gas phase diffusion corrections for the 100-200 $\mu \mathrm{m}$ droplets used in the droplet train flow reactor or they may reflect larger than anticipated uptake due to surface reaction of surface active $\mathrm{HOCl}$ in the case of $\mathrm{HCl}$ uptake, and of surface active hydronium ions in the case of aerosol flow reactor studies of surface-active ammonia and methane sulfonic acid, both of which have significantly higher proton affinities than water.

In other notable aqueous acid uptake studies both aerosol flow reactor and wetted wall flow reactor studies indicate that the uptake of sulphuric acid vapour on sulphuric acid surfaces is $\sim 1$, and a wetted wall flow reactor study indicates that the uptake coefficient for $\mathrm{SO}_{3}$ (the anhydride of sulphuric acid vapour) on sulphuric acid surfaces is also $\sim 1$. Full references for all of these studies on aqueous acid surfaces can be found in Davidovits et al. (2006).

\section{Recommendations for further work}

See combined recommendations for water vapour and trace gas uptake at the end of Sect. 5.1.7.

\subsubsection{Stable trace gas uptake on aqueous salt solutions}

A variety of salt solutions exist in the troposphere, including both sodium halides and those with ammonium as the counter ion to inorganic and organic acids. The interactions of gas-phase species with concentrated halide ion solutions are important to marine boundary layer and snow pack/seaice chemistry. In each, there is the possibility that the concentration of halide ions at the surface is enhanced over the bulk, as indicated by both molecular dynamics simulations and electro spray ionization mass spectrometry. At low temperatures, concentrated brine may coexist with snow/ice. Surface segregation appears to be most important for the largest anions, such as $\mathrm{Br}^{-}$and $\mathrm{I}^{-}$(Finlayson-Pitts, 2003). The major 
issues for gas-uptake studies are whether the chemistry occurs at the surface or in the bulk, and whether a surface proton (hydronium ion) is active as a reagent, as is frequently the case in bulk-phase processes. Novel surface-phase chemistry not involving a proton has also been suggested (Oum et al., 1998a). The second class of salt solutions - ammonium salts- occur when strong acids, such as nitric, sulfuric or hydrochloric acids are neutralized by ammonia. This process forms common secondary inorganic aerosol components in continental atmospheres. Nitric acid is taken up rapidly into water and micron-sized halide salt droplets $(\gamma>0.2)$ at RH $>60 \%$ (Abbatt and Waschewsky, 1998). On submicron particles, loss of $\mathrm{HNO}_{3}$ occurs on deliquesced sea salt or $\mathrm{NaCl}$ particles with an uptake coefficient between 0.1 and 0.5 (Saul et al., 2006; Guimbaud et al., 2002; Stemmler et al., 2008). The chemistry has not been systematically studied on ice/brine surfaces but is expected to be equally rapid.

There are a variety of reactions that involve HOX species (where $\mathrm{X}=\mathrm{Cl}, \mathrm{Br}, \mathrm{I}$ ) interacting with halide solutions, yielding gas-phase $\mathrm{XY}$. The bulk accommodation of $\mathrm{HOBr}$ on aqueous $\mathrm{NaCl}$ has been reported to be larger than $0.2(\mathrm{Ab}-$ batt and Waschewsky, 1998) and it has been reported to be 0.6 on deliquesced $\mathrm{NaBr}$ (Wachsmuth et al., 2002), but more studies are required on other aqueous systems. The interactions of $\mathrm{HOBr}$ and $\mathrm{HOCl}$ with ice/halide brines have been studied, with initial reactive uptake coefficients greater than 0.01 (Huff and Abbatt, 2000; Adams et al., 2002; Huff and Abbatt, 2002). As with $\mathrm{OH}$, there is indication that protons are not required for reactive uptake but that the chemistry is faster on acidic surfaces.

Uptake of $\mathrm{N}_{2} \mathrm{O}_{5}$ on aqueous solutions has been extensively studied. In general, the hydrolysis reaction proceeds with an uptake coefficient between 0.01 and 0.04 at high relative humidity; this value matches that on pure water surfaces. Under conditions favorable for rapid reaction of solvated $\mathrm{N}_{2} \mathrm{O}_{5}$ molecules (high $\mathrm{RH}, \mathrm{H}^{+}$catalyzed hydrolysis, high $\left[\mathrm{X}^{-}\right]$), $\gamma$ is essentially independent of RH. This strongly suggests that uptake is accommodation controlled (Fried et al., 1994; George et al., 1994, Schweitzer, 1998; Hu and Abbatt, 1997; Hanson, 1997b; Robinson et al., 1997; Hallquist et al., 2000; Hallquist et al., 2003; Thornton et al., 2003; Thornton and Abbatt, 2005a). The reaction in sulfate and nitrate salts is hydrolysis, whereas in $\mathrm{NaCl}$ and sea salt aerosols reaction leads to $\mathrm{ClNO}_{2}$ production, unless the acidity is high in which case $\mathrm{Cl}_{2}$ is formed (Roberts et al., 2008). Uptake coefficients for $\mathrm{ClNO}_{2}$ production are similar to those for $\mathrm{N}_{2} \mathrm{O}_{5}$ hydrolysis and a decrease in $\gamma$ with RH is observed (Schweitzer et al., 1998; Stewart et al., 2004; Thornton and Abbatt, 2005a). Interestingly, in the case of aqueous $\mathrm{NaCl}$, the kinetics are similar to those on pure water, and yet a different product is formed, i.e. $\mathrm{ClNO}_{2}$ and not $\mathrm{HNO}_{3}$ (Behnke et al., 1991; Thornton and Abbatt, 2005a). This implies that the initial uptake of $\mathrm{N}_{2} \mathrm{O}_{5}$ requires water molecules and is mass accommodation limited, at a value of close to 0.03 at room temperature. The differentiation of the products presumably arises further along the reaction pathway. The degree to which surface reactions compete with bulk reaction is unknown.

In neutral or weak acid solutions at $\mathrm{RH}<70 \%$ a strong trend of decreasing $\gamma$ with decreasing $\mathrm{RH}$ is observed; an abrupt drop in $\gamma$ is observed at efflorescence $\mathrm{RH}$ when phase change to solid particles occurs (Kane et al., 2001; Hallquist et al., 2003; Thornton and Abbatt, 2003). Uptake of $\mathrm{N}_{2} \mathrm{O}_{5}$ is also reduced on aerosols containing nitrate ions; as RH decreases, $\left[\mathrm{NO}_{3}^{-}\right]$increases and $\gamma$ declines proportionally due to retardation of reversible hydrolysis chemistry (Wahner et al., 1998; Mentel et al., 1999; Hallquist et al., 2003).

Since ozone is such an important atmospheric oxidant and free radical precursor the issue of potential heterogeneous atmospheric loss and surface/condensed oxidation is of interest. Ozone does not react with pure water and is only weakly soluble. This has the consequence that uptake measurements using pure water and many of its solutions are challenging due to the rapid saturation of the aqueous sample. This limitation has been addressed by the use of solutes chosen to react rapidly with dissolved ozone, eliminating the saturation problem. Uptake experiments are then analyzed (for the most part) using the resistor model:

$$
\frac{1}{\gamma_{\mathrm{obs}}}=\frac{1}{\Gamma_{\mathrm{diff}}}+\frac{1}{\alpha}+\frac{\bar{c}}{4 H R T \sqrt{k^{1} D_{l}}}
$$

where $k^{I}$ represents the pseudo-first order rate coefficient for reaction in solution and $H$ and $D_{l}$ are the Henry's law constant and the aqueous phase diffusion coefficient, in this case for ozone. In principle, plotting the inverse of the diffusioncorrected uptake coefficient vs. the inverse square root of the solution phase concentration of the aqueous reagent yields the accommodation coefficient as the intercept. This is the approach that most studies have taken. Solubility limitations of the aqueous reagent, $\mathrm{X}$, generally require a significant extrapolation to the $[\mathrm{X}]^{\frac{1}{2}} 0$ limit.

Experiments have been performed using droplet train $(\mathrm{Hu}$ et al., 1995; Magi et al., 1997), wetted wall (Utter et al., 1992; Muller and Heal, 2002a), and single-droplet (Schutze and Herrmann, 2002) techniques with halide (mainly $\mathrm{I}^{-}$) (Utter et al., 1992; Hu et al., 1995; Magi et al., 1997; Schutze and Herrmann, 2002) and sulfur oxide $\left(\mathrm{SO}_{3}^{2-}\right.$ and $\mathrm{S}_{2} \mathrm{O}_{3}^{2-}$ ) (Utter et al., 1992; Muller and Heal, 2002a) aqueous scavenger reagents. There is fairly good agreement among the measurements: all suggest that $\alpha$ could be as large as unity, though most estimate it to lie between 0.02 and 0.1 . This is consistent as well with a molecular dynamics calculation (Vieceli et al., 2005) that yielded $\alpha=0.047$.

Recently, however, it has become clear that many polarizable anions, $\mathrm{I}^{-}$in particular, may be surface active (Petersen et al., 2004; Ghosal et al., 2005; Gopalakrishnan et al., 2006; Jungwirth and Tobias, 2006; Pegram and Record, 2006). This raises the possibility that the observed ozone uptake may be influenced by surface reaction. At this date, there are no suggestions that either $\mathrm{SO}_{3}^{2-}$ or $\mathrm{S}_{2} \mathrm{O}_{3}^{2-}$ might 
act in this manner; in fact surface tension data on $\mathrm{SO}_{3}^{2-}$ indicate the opposite (Vazquez et al., 1995). One test for whether there is a surface component to the reaction is to plot $\log (\gamma)$ vs. $\log$ (excess reagent); a slope of 0.5 indicates a bulk phase reaction, whereas a unity slope shows 'pure' surface behavior. Where this procedure has been reported (Utter et al., 1992; Muller and Heal, 2002a), the sulfur oxide reagents show purely bulk reaction behavior, while the $\mathrm{Cl}^{-}$reagent appears to show a surface component to the reaction as well. The halide results are therefore suspect, because their analysis does not include the possibility of surface reaction (although in one case (Hu et al., 1995) this was considered, but it was stated that there were no indications of such an effect).

Recent laboratory results (Hunt et al., 2004; Clifford and Donaldson, 2007; Wren et al., 2010) for $\mathrm{O}_{3}$ interacting with $\mathrm{NaBr}$ suggests there may also be a surface component for $\mathrm{Br}^{-}$oxidation. The upper limit for reactive uptake coefficient of $\mathrm{O}_{3}$ loss to neutral aqueous $\mathrm{NaCl}$ is $10^{-4}$, but the mass accommodation coefficient may be larger (Abbatt and Waschewsky, 1998). There is also a need for further kinetics studies of the interactions of ozone on ice/brines, to extend an initial study of the reaction of $\mathrm{O}_{3}$ with frozen seawater that indicated bromine activation (Oum et al., 1998b).

\section{Recommendations for further work}

See combined recommendations for water vapour and trace gas uptake at the end of Sect. 5.1.7.

\subsubsection{Uptake of reactive free radical species on aqueous surfaces}

The heterogeneous uptake of three reactive radicals, $\mathrm{OH}$, $\mathrm{HO}_{2}$ and $\mathrm{NO}_{3}$, which are important tropospheric photochemistry radical products, have been studied in multiple laboratories on multiple aqueous surfaces. The material in this subsection will concentrate on these species.

Several other halogen containing reactive radicals $(\mathrm{Cl}$, $\mathrm{ClO}, \mathrm{OClO}, \mathrm{Br}, \mathrm{BrO}, \mathrm{OBrO}, \mathrm{I}, \mathrm{IO}, \mathrm{OIO}, \mathrm{IO}_{3}$ ) play a significant role in stratospheric and/or marine boundary layer chemistry, but their heterogeneous uptake processes have been much less extensively studied. Discussions of and citations for aqueous surface heterogeneous processes for some of these halogen radicals can be found in (Davidovits et al., 2006). In general definitive heterogeneous uptake studies for atmospheric halogen radicals are not available and thus the importance of their heterogeneous kinetics is not well characterized, although published studies of $\mathrm{ClO}$ and $\mathrm{BrO}$ uptake on sulphuric acid indicate that this process is too slow to be atmospherically important (Abbatt, 1996).

Initial studies of $\mathrm{HO}_{2}$ uptake onto aqueous inorganic aerosol particles revealed a relatively high value for $\alpha(\sim 0.5)$ as long bulk saturation of $\mathrm{HO}_{2}$ is avoided. This was clearly demonstrated by Mozurkewich et al., (1987), who showed the overall loss of $\mathrm{HO}_{2}$ to the particles ceased when the $\mathrm{Cu}$ (II) $\left(\mathrm{a} \mathrm{HO}_{2}\right.$ scavenger) concentration dropped below $10^{-4}$ M. Interestingly, this is also the point where the bulk reactodiffusive length for $\mathrm{HO}_{2}$ became larger than the particle radius, indicating that the uptake was consistent with bulk phase reaction. These two points illustrate an important distinction between the mass accommodation coefficient and the overall uptake coefficient, the latter often being the quantity of interest for atmospheric modelling calculations. This distinction means that if an experimentalist reports a value for $\alpha$, this value should not be included in atmospheric models until a specific loss process has been identified for that species. Later $\mathrm{HO}_{2}$ uptake studies using $\mathrm{Cu}(\mathrm{II})$ as a scavenger have confirmed the results of Mozurkewich et al. (Cooper and Abbatt, 1996; Thornton and Abbatt, 2005b; Taketani et al., 2008). For solutions that have not been doped by $\mathrm{Cu}(\mathrm{II})$, studies have been conducted on aqueous inorganic salts, such as $\mathrm{NaCl}$ and $\left(\mathrm{NH}_{4}\right)_{2} \mathrm{SO}_{4}$, and on sulfuric acid solutions. On the salt solutions, there is a loss corresponding to an uptake coefficient of roughly 0.1 , via a mechanism that is not entirely clear. Work by (Thornton and Abbatt, 2005b) at relatively high $\mathrm{HO}_{2}$ concentrations is consistent with bulk phase loss processes involving dissolved $\mathrm{HO}_{2}$ species but recent studies by Taketani et al. (2008, 2009), done at lower concentrations, are not. It is possible that a surface phase reaction is driving the kinetics. On cold, copper-free sulfuric acid solutions $(T<250 \mathrm{~K})$, a loss of $\mathrm{HO}_{2}$ on bulk liquids occurs with an uptake coefficient of $\geq 0.05$ via a mechanism that has not been identified (Hanson et al., 1992; Cooper and Abbatt, 1996). On aerosol particles the loss is smaller $(\gamma<0.01)$ at room temperature (Thornton and Abbatt, 2005b)

Hanson et al. (1992) also studied OH mass accommodation into bulk liquids and, while only relatively small lower limits of about 0.01 could be determined for water and dilute acids, uptake onto concentrated acids suggested lower limits to $\alpha$ of 0.2 . Overall these results suggest that the mass accommodation process is not rate limiting for $\mathrm{OH}$ uptake onto liquids: i.e. $\alpha>0.1$. This is consistent with recent experiments by Laskin et al. (2006), who report a lower limit of 0.1 for $\alpha$ for $\mathrm{OH}$ uptake onto deliquesced $\mathrm{NaCl}$ particles. Takami et al. (1998) report somewhat lower values for $\alpha$, ranging from 0.03 to greater than 0.1 on bulk liquid surfaces. It is not clear that mass transport in this experiment has been dealt with at a sufficiently high accuracy so that unity values for $\alpha$ can be ruled out.

For halide salt solutions reactive $\mathrm{OH}$ uptake may occur, since $\mathrm{OH}$ is known to oxidize halides in the bulk liquid phase. Reactive uptake is expected to occur at the surface given the short reacto-diffusive length predicted by bulk-phase parameters. Although the initial elementary interaction in a seawater mixture is likely with $\mathrm{Cl}^{-}$, this needs to be confirmed given the preferential enhancement of $\mathrm{Br}^{-}$at the surface. Observations show that $\mathrm{Br}^{-}$is the first species to be oxidized in $\mathrm{NaCl} / \mathrm{NaBr}$ mixtures and that halide oxidation occurs with both $\mathrm{NaCl}$ and $\mathrm{NaBr}$ solutions (Oum et al., 1998a; Frinak 
and Abbatt, 2006; Sjostedt and Abbatt, 2008). Both Oum et al. (1998a) and Sjostedt and Abbatt (2008) observe that substantial halogen formation from brines occurs at high $\mathrm{pH}$ (e.g. >7), although formation rates are higher at lower $\mathrm{pH}$.

$\mathrm{NO}_{3}$ uptake studies on liquid water revealed no limitation to the uptake process due to mass accommodation $(\alpha>0.04$ at $293 \mathrm{~K}$ ) and specific loss processes occurring in the bulk of the aqueous solution determined the overall uptake (Rudich et al., 1996). Interestingly, on pure water it was postulated a noticeable hydrolysis of $\mathrm{NO}_{3}$ produces $\mathrm{HNO}_{3}$ and the $\mathrm{OH}$ radical. The possible role of surface-active ions on the uptake rates measured for the $\mathrm{NaCl}, \mathrm{NaI}$ and $\mathrm{NaNO}_{2}$ solutions studied has not been fully assessed.

\section{Recommendations for further work}

See combined recommendations for water vapour and trace gas uptake at the end of Sect. 5.1.7.

\subsubsection{Role of organic coatings on aqueous surfaces}

The effect of organic layers on aqueous aerosol properties has been reviewed by (Donaldson and Vaida, 2006), a critical assessment of the state of current knowledge of the role that organic aerosol PM plays in atmospheric air quality and, climate change was published by (Fuzzi et al., 2006), and the adsorption and reaction of organic compounds on aqueous surfaces has been reviewed by Donaldson and Valsaraj (2010). Recent work by Pöschl, Rudich and Ammann (PRA) offers a way of treating the complex chemical processes of multicomponent aerosol systems within a coherent framework, using the example of $\mathrm{N}_{2} \mathrm{O}_{5}$ reactive uptake (Ammann and Pöschl, 2007; Pöschl et al., 2007).

There is an extensive body of experimental evidence that organic compounds perturb the uptake of trace gases onto aqueous surfaces. In the case of $\mathrm{N}_{2} \mathrm{O}_{5}$, (Folkers et al., 2003) showed that uptake by inorganic sulfate aerosol was slowed when products of the oxidation $\alpha$-pinene had condensed onto the aerosol surface. In a subsequent study, (Badger et al., 2006) showed that uptake of $\mathrm{N}_{2} \mathrm{O}_{5}$ by sulfate aerosol was reduced by humic acid. Uptake coefficients decreased by an order of magnitude as the humic acid mass fraction was increased over the range $0-50 \%$.

The effect of monolayer coatings of organic acids has been studied by Thornton and Abbatt (2005a) and McNeill et al. (2006b). Hexanoic acid coatings of aqueous $\mathrm{NaCl}$ and sea-salt surfaces inhibited both the uptake of $\mathrm{N}_{2} \mathrm{O}_{5}$ and the rate of release of the $\mathrm{ClNO}_{2}$ hydrolysis product. The effect of films of sodium dodecyl sulphate and oleic acid on uptake of $\mathrm{N}_{2} \mathrm{O}_{5}$ supports the view that monolayer coverage is sufficient to inhibit uptake (McNeill et al., 2006b; IUPAC, 2009). They found that the uptake coefficient is reduced by an order of magnitude from 0.02 to 0.001 as the organic acid mass content was increased over the range $0.05-3 \%$ by weight.
Above $3 \mathrm{wt} \%$ organic acid, no further decrease in uptake coefficient was observed. The results were interpreted in terms of a monolayer formation at $\sim 3 \mathrm{wt} \%$ oleic acid.

Molecular beam studies by Nathanson and co-workers (Park et al., 2007) have shown that the efficiency of conversion of $\mathrm{N}_{2} \mathrm{O}_{5}$ into nitric acid, $\mathrm{HNO}_{3}$, by concentrated sulphuric acid surfaces was reduced by films of butanol and hexanol. In contrast to previous work, the uptake coefficient decreased linearly from 0.12 to 0.05 with increasing surface coverage over the range $0.2-0.6$ monolayers, while earlier work in the same group showed that butanol surface layers actually enhanced the uptake and isotopic exchange of $\mathrm{HCl}$ and $\mathrm{HBr}$ with deuterated sulphuric acid surfaces (Lawrence et al., 2005b), presumably because the butanol $\mathrm{OH}$ groups enhance surface bonding sites for the halogen halide species.

Although the effect of the films on $\mathrm{N}_{2} \mathrm{O}_{5}$ uptake is undisputed, the reason for the reduction remains unclear. It may be that the organic film fundamentally inhibits the transfer of $\mathrm{N}_{2} \mathrm{O}_{5}$ across the surface, but it may also be that the organic layer merely reduces the availability of surface water, which is needed to accommodate the incoming trace gas. Anttila et al., (2006) propose that the effect of larger mass fractions on $\mathrm{N}_{2} \mathrm{O}_{5}$ uptake is better understood in terms of the combined effects of bulk phase solubility, diffusion and accommodation of the trace gas in an organic aerosol component.

Ammann and co-workers (Stemmler et al., 2008) have studied the uptake of nitric acid, $\mathrm{HNO}_{3}$, by sea-salt aerosol coated with long-chain fatty acids. The effect of surface composition was studied by varying the chain length and degree of saturation of the fatty acids used to coat the aerosol. C15 acid coatings slowed uptake by an order of magnitude, while C12 and C9 coatings had no effect. It is noteworthy that a monounsaturated $\mathrm{C} 18$ acid, oleic acid, had no effect on uptake while a saturated $\mathrm{C} 18$ acid reduced uptake. The study demonstrated that the morphology of the surface layer was important to uptake - the authors conclude that it is not the amount of fatty acid, but the phase state that is critical to uptake. The surface of aerosol particles coated with an expanded liquid monolayer films contains a significant amount of available surface water. In contrast, the condensed monolayers not only have a reduced amount of surface water, reducing the extent of reaction, but which may also inhibit transfer of reactants across the interface.

Donaldson and co-workers (Mmereki et al., 2003; Clifford et al., 2007) have shown that even sub-monolayer coatings of organic films may perturb uptake of gas phase species to the aqueous surface. Mmereki et al. (2003) demonstrated that such coatings could increase the uptake coefficient of hydrophobic compounds to the water surface, while Clifford et al. (2007) showed that inorganic acid hydrolysis is influenced by 1-octanol films. Sub-monolayer coverage of the alcohol inhibited the rate of nitric acid uptake, possibly due to a reduction of water in the interfacial region that delays hydrolysis until adsorbed acid molecules are transported closer to or into the bulk. Similar results were observed for ammonia, 
and the observations for the two species were successfully rationalized on the basis of the dependence of the rate of surface reaction on the availability of surface water. No suppression of uptake was observed in the case of butanol films, as these form a more openly packed film presumably with readily available surface water.

McNeill et al. (2008) studied the uptake of $\mathrm{OH}$ onto aqueous and solid $\mathrm{NaCl}$ particles coated with palmitic acid. Uptake onto coated solid particles was more efficient $(\gamma=$ $\left.0.3_{-0.05}^{+0.1}\right)$ than onto coated aqueous particles $(\gamma=0.05 \pm$ 0.01 ), perhaps due to a change in orientation of the palmitic acid molecules within the film and a less favourable orientation involving presentation of a less reactive (primary) carbon site towards the incoming $\mathrm{OH}$ molecules.

While difficult to relate to atmospheric conditions, surfactant films have been shown to reduce the rate of evaporation of water from an inorganic salt using the optical tweezers technique (Buajarern et al., 2007). However, butanol coatings at up to $80 \%$ monolayer coverage do not seem to inhibit the evaporation of water vapour from cold sulphuric acid surfaces (Lawrence et al., 2005a).

\section{Recommendations for further work}

Three major experimental challenges for measuring the uptake kinetics of water vapour and trace gases on pure water and aqueous solutions need further investigation. First, it is necessary to ensure that the experimental techniques that use large or moderate surface areas (e.g. wetted wall flow reactors, droplet train flow reactors, coaxial liquid jet reactors) do not underestimate uptake coefficients by improperly accounting for near surface gradients in trace gas concentrations due to finite gas phase diffusion rates. Second, it must be established that experiments that often approximate pure water uptake by employing acid or salt aqueous solutions to create and stabilize small droplets and achieve high surface to volume ratios (aerosol flow reactors, aerosol chambers) and techniques that dope aqueous solutions to overcome bulk Henry's law solubility constraints do not suffer from enhanced surface reaction and/or surface adsorption that mimic bulk mass accommodation. Third, techniques that involve rapid droplet growth (e.g. expansion cloud chambers) or rapid droplet evaporation (e.g. injection of free jets or droplet trains into near vacuum environments) must assure that their measurement and/or analysis methods accurately account for both the rapid interfacial mass transport and heat flux rates involved and that their experimental interfacial structural properties are not significantly different from the near equilibrium interfacial surfaces that are relevant for atmospheric gas uptake and evaporation processes.

Generally, reliable determination of uptake and accommodation coefficients requires approaches that, on one hand, decouple the thermodynamic equilibrium properties from kinetic properties and, on the other hand, account for the si- multaneous mass and heat transfer related to condensation and evaporation. From an experimental perspective, welldefined gas phase composition and temperature profiles are among the key factors defining useful approaches.

Measurements involving strong aqueous acid and halide salt solutions are particularly prone to the competition between interfacial reaction/adsorption and bulk liquid reaction/absorption processes and need to be carefully designed to avoid confusion. Measurement of reacto-diffusive lengths of aqueous-phase processes are needed to determine whether reactions are dominated by either surface- or bulk-phase chemistry (Hanson and Lovejoy, 1995). This is especially true for processes involving $\mathrm{Br}^{-}$and $\mathrm{I}^{-}$. Uptake studies should consider the role of high ionic strength solutions by varying the bulk ion concentrations, and they should attempt to better define interfacial ion concentrations. In the case of ice/brines, attention should be given to the phase of the substrate and the degree of surface segregation of the reactants, which will depend on temperature, overall substrate composition, and mode of surface preparation.

Since most aqueous atmospheric aerosol particles are known to contain organic components, the impact of organic surface and bulk species on their formation and evolution is a key topic for further study. The effect of organic surface films on the uptake of a wider range of trace gases, including other organics, should be determined. Further studies should also examine the effect of surface concentration and chemical content of organic films, as well as the effect of layer thickness of surface-active compounds on uptake. If possible, surface structure should be determined. Continued development of the PRA model or equivalent approaches is required to reliably represent the impact of organic films on trace gas surface adsorption, bulk absorption and the production and subsequent evaporation of both surface and bulk phase reactions and to guide the development of accurate atmospheric models that include organic aerosol effects.

The uptake of $\mathrm{H}_{2} \mathrm{O}_{2}$ offers another benchmark system for future study. There are indications that its reactivity towards aerosol is strongly dependent on both relative humidity and aerosol composition (Pradhan et al., 2010). In addition, the bulk aqueous kinetics of this system are comparatively well understood and will provide an important point of contact between the experimental community and kinetic modeling frameworks.

We also recommend that attention be increasingly given to measurements in the field that can be used to demonstrate heterogeneous chemistry on real aerosol particles. In that regard, we highlight the early, ground-breaking efforts to measure the uptake coefficients of $\mathrm{N}_{2} \mathrm{O}_{5}$ by tropospheric aerosol (Brown et al., 2006; Bertram et al., 2009) and the first attempts to measure the effects of $\mathrm{OH}$ heterogeneous oxidation of tropospheric particulates/droplets (George et al., 2008). In particular, combined studies of trace gas removal and particulate composition will allow the partitioning of trace gases and their products to be better quantified. There is also a 
need for an increased input from the field measurement community to better quantify the composition and mixing state of the atmospheric aerosol particles.

While, as detailed in Sect. 5.1.2 above, it is undoubtedly difficult to directly infer mass accommodation coefficient of water vapour on pure water by reconciling field measurements of cloud droplet number with $\mathrm{CCN}$ using conventional coupled models of mass and heat flux, more comprehensive efforts now offer promise to quantify kinetic limitations. Mapping of offline molecular level quantification of aerosol composition onto online size-resolved broad composition measurements, along with measurements of mixing state by hygroscopicity can all be provided by modern comprehensive experiments. Such data can provide input for a model including other potential limitations on the kinetics of condensation (possible film formation at a known organic composition, for example). Careful measurements of droplet microphysics and updraft velocity together with selection of case study clouds eliminating non-adiabatic conditions have the promise to provide meaningful constraints on the kinetic parameters included in such a model.

\subsection{Results and challenges for solid PM surfaces}

A wide range of inorganic, organic and mixed solid materials can be found in atmospheric PM. The solid surfaces considered for atmospheric studies, as listed in the introduction to the evaluation heterogeneous processes on the IUPAC website at University of Cambridge, UK (http://www. iupac-kinetic.ch.cam.ac.uk/), are: ice (usually in polycrystalline form); NAT (nitric acid trihydrate), NAD (nitric acid dihydrate) and SAT (sulphuric acid tetrahydrate); mineral oxides (as on naturally occurring dusts); soot (usually amorphous black carbon); and solid electrolyte salts below their efflorescence temperature. The IUPAC evaluation provides recommendations for uptake coefficients on these surfaces, except for soot and solid salts. A monograph covering much of this material has recently been published in Atmospheric Chemistry and Physics (Crowley et al., 2010). In this section trace gas uptake on a series of model systems representing the range of real PM solid surfaces and their individual components will be reviewed and outstanding challenges will be identified.

\subsubsection{Trace gas uptake on ice surfaces}

In the polar stratosphere solid "ice" occurs as Type II (pure $\mathrm{H}_{2} \mathrm{O}$ ice) and Type Ia (nitric acid trihydrate or NAT) Polar Stratospheric Clouds (PSCs) whereas it mainly occurs as cirrus (largely $\mathrm{H}_{2} \mathrm{O}$ ice) clouds in the upper troposphere as well as aviation contrails. Other forms such as metastable nitric acid dihydrate (NAD), sulphuric acid tetrahydrate (SAT) and other hydrates may also exist (Wooldridge et al., 1995). Surface properties, including structural parameters, surface disorder and the presence of mixed-phases on the surface of at- mospheric ice particles are probably more important for trace gas uptake than hitherto thought (McNeill et al., 2007).

Specific kinetic and thermodynamic data for many systems involving ice may be found in evaluations/compilations of the IUPAC Subcommittee and the NASA-JPL Panel (NASA, 2006; IUPAC, 2009).

Pure $\mathrm{H}_{2} \mathrm{O}$ ice has a structurally-disordered "quasi-liquid layer" (QLL) in the range 233-273 K whose depth depends on temperature and which may be described by an order parameter to which different measurements (Nuclear Magnetic Resonance, glancing X-ray spectroscopy, Second Harmonic Generation, etc.) are sensitive to different extents, e.g. Wei et al. (2001). A concise summary of ice properties can be found in (Abbatt, 2003).

The surface mass accommodation coefficient, $\alpha$, of $\mathrm{H}_{2} \mathrm{O}$ vapour onto pure ice is temperature dependent, measured as unity at $130 \mathrm{~K}$ and decreasing with increasing temperature down to values of $\alpha=0.12 \mathrm{~K}$ at $220 \mathrm{~K}$ for vapour-condensed ice (Delval and Rossi, 2004). A recent measurement of the average value of $\alpha$ at $223 \mathrm{~K}$ using levitated cirrus-like ice crystals (Magee et al., 2006) found $\alpha=6 \times 10^{-3}$, which represents the lowest value obtained to date. Starting at $230 \mathrm{~K} \alpha$ seems to increase with temperature in order to attain a value of $\alpha=0.7(+0.3,-0.1)$ in the range $259-273 \mathrm{~K}$ (Lu et al., 2006) for vapor-condensed ice.

The mass accommodation coefficient of water vapour depends strongly (up to a factor of 4.5) on the type of ice in the range 130 to $220 \mathrm{~K}$. This range is comparable to the temperature variation of $\alpha$ over the range $130-230 \mathrm{~K}$. It is lowest for artificial (dendritic) snow and quasi-single crystal ice and highest for vapor-condensed ice (Pratte et al., 2006). This span is even larger (a factor of 6.5) when results for uptake by cubic ice over the range of 130-140 K are included. The negative $T$-dependence and the "break" at $193 \pm 2 \mathrm{~K}$ where the negative activation energy changes from a low $(-1.25 \mathrm{~kJ} / \mathrm{mol})$ to a high $(-6.3 \mathrm{~kJ} / \mathrm{mol})$ value suggest a complex reaction mechanism involving several elementary steps and one or more precursors (Delval and Rossi, 2004; Pratte et al., 2006). Despite this complexity, measured rates of condensation are first order in $\mathrm{H}_{2} \mathrm{O}$ and rates of evaporation are zero order under all conditions examined. In addition, the negative $T$-dependence of $\alpha$ changes into a positive one at $T=230 \pm 5 \mathrm{~K}$, approximately coincident with the incipient formation of a QLL at $T=233 \mathrm{~K}$ for pure ice.

The solubility of most trace gases including acidic gases such as $\mathrm{HCl}$ and $\mathrm{HNO}_{3}$, whose solubilities in liquid $\mathrm{H}_{2} \mathrm{O}$ are high, are, in fact, rather low in ice (Hanson and Mauersberger, 1990; Thibert and Dominé, 1998). However, the interaction between pure ice and $\mathrm{HCl}$ leads to a perturbed structure at the gas-condensed phase interface that has implications for heterogeneous chemical reactions. McNeill et al. (2006a; 2007) and Aguzzi et al. (2003) established the formation of a "quasi-liquid layer" (QLL) or of an "interface region", respectively, ranging from several tens to $200 \mathrm{~nm}$ thickness at $200 \mathrm{~K}$ that enabled the practically 
unlimited uptake of $\mathrm{HCl}$ in contrast to the limited uptake on crystalline ice. Advanced quantum-chemical methods fail to reproduce the structure and depth of the QLL of ice whose structure is perturbed owing to contamination by acidic trace gases (Girardet and Toubin, 2001).

The interaction of acidic trace gases $\mathrm{HX}\left(\mathrm{X}=\mathrm{Cl}, \mathrm{Br}, \mathrm{NO}_{3}\right)$ with ice, especially of $\mathrm{HCl}$, has been studied extensively (see Huthwelker et al., 2006 for an extensive review) because of the importance of the availability of $\mathrm{HX}$ at the gas-ice interface for effectively bimolecular chlorine activation processes such as reactions:

$\mathrm{ClONO}_{2}+\mathrm{HCl}($ ads $) \rightarrow \mathrm{Cl}_{2}+\mathrm{HNO}_{3}$ (ads)

$\mathrm{N}_{2} \mathrm{O}_{5}+\mathrm{HCl}($ ads $) \rightarrow \mathrm{ClNO}_{2}+\mathrm{HNO}_{3}$ (ads)

The mechanism of these reactions requires the presence of $\mathrm{H}_{3} \mathrm{O}^{+}$and $\mathrm{Cl}^{-}$ions resulting from electrolytic dissociation of $\mathrm{HCl}$ adsorbed on ice. The chloride ion, $\mathrm{Cl}^{-}$, induces a nucleophilic displacement of nitrate ion, $\mathrm{NO}_{3}^{-}$, resulting in volatile $\mathrm{Cl}_{2}$ :

$\mathrm{Cl}^{-}+\mathrm{ClONO}_{2} \rightarrow \mathrm{Cl}_{2}+\mathrm{NO}_{3}^{-}$(ads)

The low activation energy of this ionic displacement reaction is the mechanistic reason for the high rate of these reactions, even at stratospheric temperatures; in contrast to homogeneous (non-ionic) gas phase processes.

When $\mathrm{HCl}$ is exposed to $\mathrm{H}_{2} \mathrm{O}$ ice the spontaneous decay of $\mathrm{HCl}$ on ice into $\mathrm{H}_{3} \mathrm{O}^{+}+\mathrm{Cl}^{-}$at $T>90 \mathrm{~K}$ is observed by using $\mathrm{X}$-ray absorption, a surface-sensitive detection technique (Parent and Laffon, 2005). Second harmonic generation (Henson et al., 2004), Rutherford back scattering (Krieger et al., 2002), ellipsometry (McNeill et al., 2006a) and many other experimental methods point to the existence of both crystalline $\left(\mathrm{HCl}^{6} \mathrm{H}_{2} \mathrm{O}, \mathrm{HCl}_{3} \mathrm{H}_{2} \mathrm{O}\right)$ as well as amorphous phases of potential atmospheric importance (Zondlo et al., 2000). The unstructured ice stability region of the $\mathrm{HCl} / \mathrm{H}_{2} \mathrm{O}$ phase diagram reveals an amorphous region around the "ice"/liquid coexistence phase boundary as opposed to the interior ice stability region that contains crystalline ice with $\mathrm{HCl}$ "impurities". The experimental distinction between a QLL, a structurally perturbed region induced by $\mathrm{HCl}$ adsorption on ice, and the crystalline region of "ice" in the interior of the $\mathrm{HCl} / \mathrm{H}_{2} \mathrm{O}$ phase diagram results in an important difference in reactivity of the ice phase under atmospheric conditions, depending on $T$ and the partial pressure of $\mathrm{HCl}$ (McNeill et al., 2006a; 2007). In addition to the ionic nature of $\mathrm{HCl}$ the structural properties of the ice matrix control the rate of chlorine activation: the QLL supports continuous $\mathrm{HCl}$ uptake in contrast to the crystalline region where uptake saturates at a formal monolayer or so. For $\mathrm{HBr}$ no phase diagram exists for atmospheric conditions, but FTIR absorption spectra in the $\mathrm{HBr} / \mathrm{H}_{2} \mathrm{O}$ system suggest the existence of an amorphous phase at the expense of crystalline hydrates.
For nitric acid several hydrates are known at atmospheric temperatures and as characterized in a series of published phase diagrams (Worsnop et al., 1993). In addition, amorphous $\mathrm{HNO}_{3} / \mathrm{H}_{2} \mathrm{O}$ phases are known to nucleate, albeit inefficiently, to the corresponding crystalline phases (Zondlo et al., 2000), in contrast to $\mathrm{HCl}$ hexahydrate that does not crystallize at atmospheric conditions (Wooldridge et al., 1995). However, nucleation of NAT and NAD on ice is slow and occurs only at high supersaturation ratios of $\mathrm{H}_{2} \mathrm{O}$ vapour relative to the corresponding hydrates. Similar to $\mathrm{HCl} / \mathrm{H}_{2} \mathrm{O}$, the uptake coefficient of $\mathrm{HNO}_{3}$ on ice has a negative $T$ dependence with multilayer uptake at $T<205 \mathrm{~K}$, probably because of substantial surface modification, and with (sub)monolayer-uptake behavior above that temperature (Aguzzi and Rossi, 2001; Ullerstam et al., 2005). There is some disagreement about the temperature dependence of the $\mathrm{HNO}_{3}$ uptake coefficient, as described in (Ullerstam et al., 2005).

Typical halogen exchange reactions, as discussed above, occur between adsorbed $\mathrm{HCl}$ and $\mathrm{ClONO}_{2}, \mathrm{BrONO}_{2}$ and $\mathrm{N}_{2} \mathrm{O}_{5}$ as a nucleophilic displacement by $\mathrm{Cl}^{-}$at the ice interface. These effectively bimolecular reactions are extremely efficient around $200 \mathrm{~K}$ because of the availability of chloride and bromide at the interface (Oppliger et al., 1997; Aguzzi et al., 2003; McNeill et al., 2006a). The hydrolysis mechanism of $\mathrm{N}_{2} \mathrm{O}_{5}$ is expected to be acid catalyzed in analogy to solution studies, yet its hydrolysis rate does not seem to be influenced by the presence of $\mathrm{HNO}_{3}$, its hydrolysis product. The rate of hydrolysis is essentially the same on both ice and sulphuric acid substrates (see IUPAC (2009) and NASA (2006)) at nominally high acidity. Hydrolysis of $\mathrm{ClONO}_{2}$ on ice in the absence of HX is slow and prone to saturation, whereas that of $\mathrm{BrONO}_{2}$ is significantly faster. The solubility of $\mathrm{HOCl}$ in ice/ $\mathrm{HNO}_{3}$, the latter being the other $\mathrm{ClONO}_{2}$ hydrolysis product, is small whereas that for $\mathrm{HOBr}$ from $\mathrm{BrONO}_{2}$ hydrolysis is appreciably larger and leads to a sustained vapour pressure of $\mathrm{HOBr}$ over ice $/ \mathrm{HNO}_{3}$.

The evaporative lifetime of complex atmospheric ice particles is longer than hitherto believed owing to the slower than expected evaporation rate of $\mathrm{H}_{2} \mathrm{O}$ in the presence of $\mathrm{HCl}$ and $\mathrm{HNO}_{3}$ (Livingston and George, 1998; Delval et al., 2003). The decrease of the absolute rate of $\mathrm{H}_{2} \mathrm{O}$ evaporation relative to pure $\mathrm{H}_{2} \mathrm{O}$ ice is a function of the amount of adsorbed trace gas and the structure of the trace gas hydrate being either in an amorphous or crystalline state. This decrease in evaporation rate may be as high as a factor of ten or more, depending on the amount of $\mathrm{HCl}$ and $\mathrm{HNO}_{3}$ on the ice. Thermodynamically efficient sealing or "capping" of a pure ice phase under a crystalline coating of an acid hydrate has not been observed for thin films in the $\mu \mathrm{m}$ thickness range (Biermann et al., 1998; Delval et al., 2003). The component with the higher vapour pressure and/or the faster evaporation rate always finds its way across a crystalline or amorphous overlayer of lower vapour pressure and/or evaporation rate in order to preferentially evaporate from the condensed phase. Therefore, "burying" of a high-vapour pressure ice 
phase under a low volatility "blanket" has not been observed to date for a $\mu \mathrm{m}$-size overlayer thickness.

Weak acids such as $\mathrm{SO}_{2}$ adsorb onto ice after electrolytic dissociation to $\mathrm{HSO}_{3}^{-}$(Clegg and Abbatt, 2001). As expected, the uptake is inhibited and increased by acidic and basic adsorbates on the ice, respectively. Unlike most other adsorbed species on ice, the surface coverage of $\mathrm{SO}_{2}$ shows a positive $T$-dependence together with $\mathrm{CO}_{2}$ (Abbatt, 2003). Other weak acids such as $\mathrm{HOCl}, \mathrm{HOBr}$ and $\mathrm{HONO}$ have been studied as well. The case of $\mathrm{HOBr}$ is interesting because $\gamma$ has a sizable negative $T$-dependence suggesting possible compound (hydrate) formation on ice. The interaction of oxygenated organic species such as alcohols, ketones, aldehydes and organic acids are distinctly different from that of strong acids and are well described by a nondissociative Langmuir model associated with a monolayer-like surface coverage for a remarkably wide range of molecules (Abbatt, 2003; Hessberg et al., 2008). Hydrogen bonding is thought to be the dominant interaction and most adsorption enthalpies are larger than those for gas-liquid condensation, thus pointing towards a strong adsorptive interaction. Adsorption enthalpies are also lower than energies of solvation, indicating that these adsorbed molecules do not acquire a full hydration shell.

Gao et al. (2004) present field data on $\mathrm{H}_{2} \mathrm{O}$ and $\mathrm{HNO}_{3}$ in the free troposphere and conclude that certain atmospheric strata within the flight path of the aircraft measurement platform are supersaturated in $\mathrm{H}_{2} \mathrm{O}$ vapour up to $130 \% \mathrm{RH}$. Invoking the presence of cubic ice decreases the degree of supersaturation to $120 \% \mathrm{RH}$, which is still insufficient to explain the high supersaturation ratios (Peter et al., 2006; Shilling et al., 2006). The answer may in part lie in the existence of a kinetic effect; the approach of the $\mathrm{H}_{2} \mathrm{O}$ partial pressure to equilibrium saturation vapour pressures may take substantially longer in agreement with low measured values of $\alpha\left(\mathrm{H}_{2} \mathrm{O}\right)$ or decreases in the evaporation rate owing to adsorption of acidic trace gases (HX) onto ice surfaces.

\section{Recommendations for further work}

Several aspects of trace gas interactions with ice remain unexplored. At $T>193 \pm 2 \mathrm{~K} \alpha$ for $\mathrm{H}_{2} \mathrm{O}$ vapor interacting with ice has a small but significant negative $T$-dependence of approximately $-6.3 \mathrm{~kJ} / \mathrm{mol}$. The work of Sadtchenko and others (IUPAC 2009) report an $\alpha$ value close to, but significantly less than, 1.0 in the range $258-273 \mathrm{~K}$. However, the $T$-dependence in the intermediate range $220-260 \mathrm{~K}$ remains unknown. It is currently not known how the $\alpha$ values for ice and liquid $\mathrm{H}_{2} \mathrm{O}$ "connect" at the junction solid/liquid near $273 \mathrm{~K}$, except for the work of Wei et al. (2001). Measurements of $\alpha$ on liquid $\mathrm{H}_{2} \mathrm{O}$ (including a small supercooled range) suggest a negative $T$-dependence as well, which is in contrast to the constant $T$-independent value for ice reported by Lu et al. (2006).
In view of the importance of heterogeneous atmospheric reactions involving bromine it is desirable to obtain knowledge of relevant portions of the $\mathrm{HBr} / \mathrm{H}_{2} \mathrm{O}$ phase diagram and the ability of the "ice" phase to take up $\mathrm{HBr}$ and other bromine-containing species such as $\mathrm{HOBr}$ for atmospheric conditions. Similarly, it is not known if the "ice" region of the $\mathrm{HNO}_{3} / \mathrm{H}_{2} \mathrm{O}$ phase diagram is subdivided into an amorphous (QLL) and a crystalline domain corresponding to two different reactivities akin to the case of $\mathrm{HCl} / \mathrm{H}_{2} \mathrm{O}$.

Water-rich" vs. " $\mathrm{HNO}_{3}$-rich" or "HCl-rich" phases of the crystalline $\mathrm{HNO}_{3}$ - and $\mathrm{HCl}$ hydrates are expected on the basis of the corresponding phase diagrams. In contrast, spectroscopic studies (FTIR in transmission and grazing incidence) point towards a single identifiable crystalline hydrate in addition to "excess" $\mathrm{H}_{2} \mathrm{O}$ (ice). Evaporation experiments of mixed ice/NAT or ice/ $\mathrm{HCl}$ hexahydrate distinguish between a pure ice phase at the characteristic ice evaporation rate and a phase whose evaporation rate continuously decreases with decreasing $\mathrm{H}_{2} \mathrm{O}$ content. The properties of the interface including the change of its molar composition upon evaporation are insufficiently known.

In many of the earlier studies on the heterogeneous reactivity of trace gases on contaminated ice surfaces the identity of the substrates was only identified by its vapour pressure, which seems insufficient for positive identification of binary or ternary solid phases. Many of these uptake and evaporation experiments should be repeated where the type of substrate should be identified using spectroscopic techniques. There is no proof that crystalline hydrates were indeed nucleated before trace gas uptake in cases where it was claimed that uptake occurred on these hydrates. With the benefit of hindsight we expect that many of these uptake experiments have been performed on solids that were ill defined. Systematic uptake experiments of $\mathrm{H}_{2} \mathrm{O}$ and atmospheric trace gases on well-defined ice substrates should be undertaken over temperature ranges of atmospheric interest.

Uptake experiments on ice particles of 0.5 to $10 \mu \mathrm{m}$ diameters at atmospheric temperatures should be performed in order to confirm laboratory results obtained on macroscopic ice coatings or thin solid ice films. This would help determine to what extent laboratory results may be transferred to atmospheric ice PM.

The gas-condensed phase interface of trace gas hydrates should be characterized in multi-diagnostic experiments simultaneously probing both phases under identical experimental conditions. The role of amorphous and crystalline ice in the "ice" domain of binary and ternary phase diagrams should be explored to determine the reactivity and chemical potential of $\mathrm{H}_{2} \mathrm{O}$ at the interface. Investigations of the uptake kinetics/dynamics of trace gases including $\mathrm{H}_{2} \mathrm{O}$ vapour on well-characterized trace gas hydrate/ice mixtures should also be performed. 


\subsubsection{Trace gas uptake on mineral and mineral dust surfaces}

Mineral dust is an important factor in atmospheric chemistry owing to its direct (scattering and absorption) and indirect radiative effects (important ice nucleating abilities) (Archuleta et al., 2005). In addition, the presence of mineral dust may change the atmospheric composition owing to the occurrence of fast heterogeneous reactions of atmospheric trace gases: $\mathrm{H}_{2} \mathrm{O}, \mathrm{HNO}_{3}, \mathrm{~N}_{2} \mathrm{O}_{5}, \mathrm{NO}_{3}, \mathrm{SO}_{2}, \mathrm{O}_{3}, \mathrm{NO}_{2}$. Mineral surfaces can also adsorb a wide range of organic species (Falkovich and Rudich, 2001; Falkovich et al., 2004). All atmospheric mineral dust contains variable amounts of $\mathrm{CaCO}_{3}$ as well as a range of inorganic oxides (Usher et al., 2003). All of the above trace gases interact with both mineral dust surrogates (Saharan dust, Arizona test dust, China loess, etc.) or pure mineral dust model compounds $\left(\mathrm{CaCO}_{3}, \mathrm{Al}_{2} \mathrm{O}_{3}, \mathrm{SiO}_{2}\right.$, $\mathrm{CaO}, \mathrm{Fe}_{2} \mathrm{O}_{3}$, etc.) either by: A) forming adsorbates (nitrates, sulfites, sulfates) on substrates with unreactive bulk phases (Ullerstam et al., 2003; Seisel et al., 2004); or B) volatile reaction products due to acid-base reactions of acidic atmospheric trace gases $\left(\mathrm{HNO}_{3}, \mathrm{HCl}, \mathrm{SO}_{2}\right)$ on $\mathrm{CaCO}_{3}$ (Santschi and Rossi, 2006).

(A) $\mathrm{MOOH}+\mathrm{HNO}_{3} \rightarrow \mathrm{MO} \cdot \mathrm{NO}_{3}+\mathrm{H}_{2} \mathrm{O}$ (Seisel et al., 2004)

(B) (a) $\mathrm{Ca}(\mathrm{OH})\left(\mathrm{HCO}_{3}\right)+\mathrm{HX}\left(\mathrm{X}=\mathrm{NO}_{3}, \mathrm{Cl}\right)$

$\rightarrow \mathrm{Ca}(\mathrm{X})\left(\mathrm{HCO}_{3}\right)+\mathrm{H}_{2} \mathrm{O}$

(b) $\mathrm{Ca}(\mathrm{X})\left(\mathrm{HCO}_{3}\right)+\mathrm{HX} \rightarrow \mathrm{Ca}(\mathrm{X})_{2}+\mathrm{H}_{2} \mathrm{O}+\mathrm{CO}_{2}$

where $\mathrm{MOOH}$ represents the mineral dust (oxide) interface.

Knowledge of the dependence of heterogeneous kinetic parameters on relative humidity is required to translate laboratory results to atmospheric conditions. As pointed out in Sect. 3, coated wall and Knudsen flow reactor studies are often unable to adequately address this important question, in contrast to aerosol flow reactor and static reaction chamber experiments where $\mathrm{RH}$ variations are easier to investigate. Generally, the dependence of $\gamma$ on relative humidity is not dramatic for atmospherically relevant values in the range of 30 to $95 \% \mathrm{RH}$, with $\gamma$ generally varying less than a factor of three over that $\mathrm{RH}$ range. The work of Mogili et al. (2006a), Wagner et al. (2008, 2009), Sullivan et al. (2009), and Vlasenko et al. (2009) present representative examples of this modest effect. Both increases of $\gamma$ with RH (Liu et al., 2008) as well as decreases (Mogili et al., 2006a) have been observed. This has been interpreted in terms of competing effects of $\mathrm{H}_{2} \mathrm{O}$ vapor adsorption and ionic solvation by adsorbed water on the surface of the mineral dust particles (Vlasenko et al., 2009). The effect of RH on $\gamma$ must be evaluated on a case-by-case basis in order to obtain a reliable functional dependence of $\gamma$ on this important atmospheric parameter.
The interaction of $\mathrm{NO}_{2}$ and $\mathrm{N}_{2} \mathrm{O}_{5}$ with mineral dust leads to the formation of surface nitrates (Usher et al., 2003) and variable amounts of $\mathrm{HNO}_{3}$, respectively (Karagulian et al., 2006). Surface reaction of $\mathrm{HNO}_{3}$ leads to volatile secondary products $\mathrm{H}_{2} \mathrm{O}$ and $\mathrm{CO}_{2}$ on $\mathrm{CaCO}_{3}$ together with solid $\mathrm{Ca}\left(\mathrm{NO}_{3}\right)_{2} . \mathrm{NO}_{3}$ free radical uptake leads to the formation of $\mathrm{N}_{2} \mathrm{O}_{5}$ and $\mathrm{HNO}_{3}$ in the presence of $\mathrm{NO}_{2}$ through EleyRideal surface recombination, except on Saharan dust where only uptake of $\mathrm{NO}_{3}$, without formation of a volatile product, is observed (Karagulian and Rossi, 2005). $\mathrm{SO}_{2}$ leads to the formation of surface sulfites and sulfates under oxidizing conditions (Ullerstam et al., 2003; Usher et al., 2003), whereas it reacts on $\mathrm{CaCO}_{3}$ by releasing prompt $\mathrm{CO}_{2}$ and by delayed formation of $\mathrm{H}_{2} \mathrm{O}$ (Santschi and Rossi, 2006). $\mathrm{O}_{3}$ decomposes into $\mathrm{O}_{2}$ and a surface oxide in a non-catalytic way (Ullerstam et al., 2002; Karagulian and Rossi, 2006), at variance with results obtained by Grassian and coworkers (Usher et al., 2003) and Hanisch and Crowley (2003). All mechanisms are complex and seem to involve surface intermediates. The interaction of $\mathrm{CaCO}_{3}$ with atmospheric trace gases implies the presence of a weathered surface as represented by $\mathrm{Ca}(\mathrm{OH})\left(\mathrm{HCO}_{3}\right)$ (Stipp et al., 1994). Mechanistic studies reveal that $\mathrm{SO}_{2}$ first attacks the surface by reacting with the $\mathrm{HCO}_{3}$ group leading to prompt $\mathrm{CO}_{2}$, whereas the acidic gases $\mathrm{CO}_{2}, \mathrm{HCl}$ and $\mathrm{HNO}_{3}$ first attack the $\mathrm{OH}$-group leading to prompt $\mathrm{H}_{2} \mathrm{O}$ (Santschi and Rossi, 2006). In order to obtain a molecular understanding of interfacial chemistry the surface composition of mineral dust materials and their proxies, in addition to $\mathrm{CaCO}_{3}$, have to be taken into account. In many cases surface hydroxyl groups represented by $\mathrm{MOOH}$ (see above) are the gateways to the reactivity of atmospheric trace gases with mineral dust substrates.

Recently, laboratory studies on the reactivity of mineral dust materials and proxies have been conducted in static aerosol chambers of varying volumes in the presence of atmospherically relevant concentrations of trace gases (Cwiertny et al., 2008). The advantage of such chamber studies lies in the opportunity to study heterogeneous reactions at elevated relative humidity and ambient temperatures, which has not been possible in studies using low-pressure reactors and laminar flow coated-wall flow tubes. In addition, aerosol flow tubes operated at atmospheric pressure are inappropriate for slow, albeit significant, heterogeneous reactions that need to be studied at low reactant pressure in order to avoid excessive saturation of the mineral dust surface. Pertinent examples are the interaction of $\mathrm{O}_{3}$ with mineral dust proxies $(\alpha$ $\mathrm{Fe}_{2} \mathrm{O}_{3}, \alpha-\mathrm{Al}_{2} \mathrm{O}_{3}$ ), the reaction of $\mathrm{CH}_{3} \mathrm{COOH}$ with $\mathrm{CaCO}_{3}$, $\mathrm{H}_{2} \mathrm{O}$ uptake on clay minerals and $\mathrm{SO}_{2}$ uptake on $\alpha-\mathrm{Fe}_{2} \mathrm{O}_{3}$ (hematite) and $\alpha-\mathrm{FeOOH}$ (goethite).

Another new type of experiment has recently been developed to study the exposure of mineral dust particles to atmospheric trace gases that affect their ability to nucleate cloud liquid water and/or ice condensation. It allows examination of the cloud condensation properties of aerosols processed under controlled trace gas exposure, either in an aerosol flow 
tube (Sullivan et al., 2009) or in an aerosol chamber (Möhler et al., 2008). The results are expressed in terms of parameters that determine the time scale of cloud condensation as a function of $\mathrm{H}_{2} \mathrm{O}$ vapor supersaturation and exposure to atmospheric trace gases, either with a CCNC (cloud condensation nucleus counter) (Sullivan et al., 2009), or in an adiabatic aerosol expansion chamber (Möhler et al., 2008). When extrapolated to atmospheric conditions, the experimental parameters enable calculation of the time scale for the transition from a non-hygroscopic to a hygroscopic aerosol particle state, or vice-versa, depending on the particle surface chemistry. The atmospheric fate and surface composition of aerosol particles also affect the radiative properties that directly impact climate change numerical predictions.

\section{Recommendations for further work}

Available laboratory data reveal several important areas for future work. For experiments where both trace gas-uptake and formation of a surface adsorbate have been studied under nominally identical experimental conditions, an important discrepancy in the uptake kinetics has often been observed. The average steady-state uptake coefficient of $\mathrm{HNO}_{3}$ on $\alpha-\mathrm{Al}_{2} \mathrm{O}_{3}$ has been measured as $\gamma_{s s}=4 \times 10^{-8}$ using the integrated absorption band of $\mathrm{HNO}_{3}$ at $1680 \mathrm{~cm}^{-1}$ (Goodman et al., 2001) whereas gas-uptake experiments yield $\gamma_{s s}=\gamma_{\mathrm{obs}}$ $=3 \times 10^{-3}$ (Underwood et al., 2001). A similar example is provided by (Ullerstam et al., 2003), where the measured uptake coefficients for $\mathrm{SO}_{2}$ interacting with Saharan dust in the presence of $\mathrm{NO}_{2}$ differ by three orders of magnitude. In these studies on mineral dust proxy surfaces uptake measurements based on gas phase loss yield larger $\gamma$ values than studies of time-dependent changes of diffuse reflectance monitored using FTIR (DRIFTS), which monitors the accumulation of surface reaction products. In some more recent studies this discrepancy in $\gamma$ values has narrowed, although significant differences remain (Mashburn et al., 2006). According to Mashburn et al. (2006) the DRIFTS-derived $\gamma$ values should be regarded as lower limiting values. Work by Adams et al. (2005) attempted to account for these observations in terms of a 2-site absorption model.

For $\mathrm{HNO}_{3}$ uptake there still is a discrepancy of four orders of magnitude in $\gamma$, depending on whether or not one applies a surface area correction due to surface pore diffusion. For example, Hanisch and Crowley $(2001 \mathrm{a} ; 2001 \mathrm{~b})$ found $\gamma_{0}$ to be independent of sample mass and derived $\gamma_{0} \sim 0.1$ on the basis of the geometric surface area of the sample. In contrast, Underwood et al. (2001) and Frinak et al. (2004) found a dependence of $\gamma_{\text {obs }}$ on sample mass and calculated a "true" value of $\gamma$, either on the basis of the measured BET surface area (lower limit) or of the pore diffusion model. In both cases the resulting $\gamma_{0}$ values were lower by several orders of magnitude. A solution to these issues is afforded by experiments in aerosol flow reactors, where the uptake coefficient on Arizona Test Dust was determined to be 0.1 (Vlasenko et al., 2006) confirming the Hanish and Crowley results.

Another striking example is provided by the $\mathrm{N}_{2} \mathrm{O}_{5} /$ Saharan dust system, examined both in a Knudsen flow reactor and in an aerosol flow tube (Wagner et al., 2008). These authors measured $\gamma=1.3 \times 10^{-2}$ at zero relative humidity in an aerosol flow tube compared with $\gamma=3.7 \times 10^{-2}$ in a Knudsen flow reactor. The agreement within a factor of three is considered to be satisfactory when taking into account that the value obtained in the Knudsen flow reactor has to be regarded as an upper limit because it was evaluated using the geometric surface area of the mineral dust. Other examples of $\mathrm{N}_{2} \mathrm{O}_{5}$ interacting with mineral dust substrates presented by Wagner et al. (2009) make a strong case for the use of the geometric surface instead of the total internal and external (BET) surface area for the normalization of the measured heterogeneous rate constant leading to $\gamma$ values. In light of this result the accuracy of $\mathrm{N}_{2} \mathrm{O}_{5}$ uptake kinetics on identical mineral dust substrates obtained by Mogili et al. (2006b) in an aerosol chamber must be questioned as the resulting $\gamma$ values are smaller by a factor of 25 and 110 compared to $\gamma$ values obtained by Wagner et al. (2009) and Karagulian et al. (2006), respectively; the discrepancy may be attributed to the use of the BET surface in the evaluation of $\gamma$ by Mogili et al. (2006) whereas the surface area in the aerosol flow tube was calculated by the aerodynamic particle diameter. In the case of quartz $\left(\mathrm{SiO}_{2}\right)$ substrates, the differences between results from Mogili et al. (2006) and Wagner et al. (2009) amount to a factor of 200 .

The quantity of surface-adsorbed $\mathrm{H}_{2} \mathrm{O}$ that controls many interfacial hydrolysis reactions, manifested by a significant humidity dependence for uptake kinetics (Vlasenko et al., 2006; $\mathrm{Li}$ et al., 2006), is uncertain by a factor of two: at 33\% RH Santschi and Rossi (2006) derive a weakly-bound $\mathrm{H}_{2} \mathrm{O}$ layer over $\mathrm{CaCO}_{3}$ of 3.5 formal monolayers, whereas Goodman et al. (2001) arrive at half that value. In contrast, only $1.4 \%$ of all $\mathrm{H}_{2} \mathrm{O}$ molecules on $\mathrm{CaCO}_{3}$ at $33 \% \mathrm{RH}$ are strongly bound, i.e. desorb at $T>480 \mathrm{~K}$ under vacuum (Santschi and Rossi, 2006). The water content of Arizona test dust and $\mathrm{CaCO}_{3}$ surfaces was determined by Gustafsson et al. (2005), using three complementary techniques whose results were in reasonable agreement.

The photochemistry of gases adsorbed onto mineral dust particles is largely unknown, especially for particles containing $\mathrm{TiO}_{2}$ (rutile or anatase). This material may lead to interfacial charge-separation processes and may support oneelectron redox chemistry of adsorbed gases upon adsorption of $\mathrm{H}_{2} \mathrm{O}$. For example, recent work employing $\mathrm{TiO}_{2}$ aerosol, mixed $\mathrm{TiO}_{2} / \mathrm{SiO}_{2}$ and authentic dusts has demonstrated an efficient route for the photochemical conversion of $\mathrm{NO}_{2}$ to HONO. $\mathrm{NO}_{2}$ uptake coefficients of the order of $10^{-3}$ were measured (Gustafsson et al., 2006; Ndour et al., 2008), much greater than has been observed in similar studies employing organic surfaces. The rate of conversion is a function of $\mathrm{RH}$, 
with a maximum value around $20 \%$, implying efficient electron transfer from the surface that is efficiently quenched by adsorbed water. However, the surface residence times of adsorbed gases must be sufficiently long so as to enable this type of chemistry.

The role of co-adsorption of trace gases onto mineral dust particles and potential synergistic effects is largely unexplored. Co-adsorption is expected to lead to a reactivity change for the gas-solid interface at high values of $\mathrm{RH}$, since, as discussed above, both oxidant and $\mathrm{H}_{2} \mathrm{O}$ vapor are competing for the same surface sites. This competition must be considered in relation to aging effects on mineral dust reactivity in polluted air masses. The loss of reactivity due to exposure to $\mathrm{O}_{3}, \mathrm{HNO}_{3}, \mathrm{SO}_{2}$ and other trace gases are important issues remaining to be elucidated, as is the rate of reactivation by exposure to $\mathrm{H}_{2} \mathrm{O}$ vapour. The same holds for the effect of surface adsorbates on the chemical reactivity and for the claimed catalytic oxidation of adsorbed sulfites to stable sulfates by air oxygen or other oxidants (e.g. $\mathrm{O}_{3}$ ). For example, a word of caution is in order regarding the catalytic heterogeneous decomposition of $\mathrm{O}_{3}$. No substrate should be regarded as catalytic until the product stoichiometry $2 \mathrm{O}_{3}->3 \mathrm{O}_{2}$ has been proven on a sustained basis over a long exposure period. Turn-over numbers based on ill-defined total (BET) surface areas offer little insight into the decomposition mechanism, in part because of questions of mass transport: the rate of exposure of these sites to gas phase $\mathrm{O}_{3}$ molecules is uncertain (Cwiertny et al., 2008).

Once the kinetics of the most important heterogeneous reactions of trace gases on mineral dust and the associated reaction mechanisms have been studied using both Knudsen and laminar coated-wall flow reactors it is recommended that the same reactions be studied using either atmospheric pressure aerosol reactors or static aerosol chambers for two reasons: (a) aerosols obviate the need for correction due to porosity effects of the solid substrate and the ability to calculate particle surface area directly from measured mobility or aerodynamic diameters, and therefore remove a potentially important bias from the kinetic results; (b) uptake experiments may be performed under atmospheric humidity conditions which may be particularly important in cases where deliquescent reaction products such as $\mathrm{Ca}\left(\mathrm{NO}_{3}\right)_{2}$ and anhydrous $\mathrm{CaCl}_{2}$ are generated from the reaction of $\mathrm{CaCO}_{3}$ with $\mathrm{HNO}_{3}$ and $\mathrm{HCl}$, respectively. Aerosol flow tube and static aerosol reactor experiments using mineral dust particles are currently being performed in several laboratories (Vlasenko et al., 2006; Preszler Prince et al., 2007; Wagner et al., 2008; Vlasenko et al., 2009; Wagner et al., 2009; Cwiertny et al., 2008). Other types of experiments that emphasize the study of the composition of the interface of the condensed phase upon trace gas uptake including free radicals (Laskin et al., 2006; Liu et al., 2008) may become important tools to study trace gas uptake on mineral dust aerosol.

The following gases are suggested for detailed study in atmospheric pressure aerosol flow reactors and static aerosol reaction chambers in the presence of characterized mineral dust aerosol over a representative range of atmospheric humidity: $\mathrm{HNO}_{3}, \mathrm{SO}_{2}\left(+\mathrm{NO}_{2}, \mathrm{O}_{2}\right.$ or $\left.\mathrm{O}_{3}\right), \mathrm{N}_{2} \mathrm{O}_{5}, \mathrm{O}_{3}, \mathrm{NO}_{3}, \mathrm{NO}_{2}$, $\mathrm{H}_{2} \mathrm{O}_{2}$ and $\mathrm{HO}_{2}$. The species in italics have previously been studied using Knudsen and laminar flow reactors (IUPAC, 2009; Crowley et al., 2010). The uptake of $\mathrm{H}_{2} \mathrm{O}_{2}$ offers another benchmark system for future study (Pradhan et al., 2010). The aqueous kinetics of this system are comparatively well understood and will provide an important point of contact between the experimental community and the Pöschl, Ammann and Rudich model framework.

\subsection{Trace gas uptake on soot surfaces}

Soot is typically composed of elemental carbon and highly functionalized carbonaceous compounds, such as saturated and unsaturated hydrocarbons, alkanoic acids, differently substituted aromatics, alcohols, ketones, dicarboxylic acids, polycyclic aromatic hydrocarbons and their derivatives, which comprise soot's organic fraction. Unfortunately, soot produced by different fuels or the same fuels burned under different combustion conditions can have rather variable chemical and morphological characteristics, so trace gas uptake and reaction parameters can vary significantly depending on the soot or soot surrogate substrate used and its history of chemical aging.

A significant body of literature describes the uptake of the abundant atmospheric oxidants $\mathrm{NO}_{2}, \mathrm{NO}_{3}, \mathrm{~N}_{2} \mathrm{O}_{5}, \mathrm{HNO}_{3}$, $\mathrm{HONO}, \mathrm{O}_{3}$ and $\mathrm{OH}$ on soot (Kleffmann et al., 1999; Longfellow et al., 2000; Stadler and Rossi, 2000; Arens et al., 2001; Bertram et al., 2001; Pöschl et al., 2001; Saathoff et al., 2001; Salgado and Rossi, 2002; Esteve et al., 2006; Karagulian and Rossi, 2007; Aubin and Abbatt, 2007; McCabe and Abbatt, 2009; Shiraiwa et al., 2009). First, soot provides a substrate for adsorption of these species on its wide range of different surface sites, as a starting point for surface reactions. The most striking common feature of these processes is that, except for $\mathrm{OH}$, uptake is characterized by relatively fast deactivation, owing to a limited number of sufficiently reactive surface species. Therefore, the role of these processes as a sink for most gas phase oxidants is probably limited. This has had a notable impact on modelling studies since it was recognized that loss of gas phase species to particles with limited uptake capacity can not be described by a simple time-independent uptake coefficient (Aumont et al., 1999; Ammann and Pöschl, 2007).

While the overall significance of trace gas uptake by soot affecting atmospheric trace gas content is still under debate, it has become evident that these trace gas/soot reactions are very significant in processing soot with respect to toxicity (e.g. Ferry et al., 2002, Lammel and Novakov, 1995; Pöschl, 2002) as well as the ability of soot to act as cloud condensation nuclei (Decesari et al., 2002; Persiantseva et al., 2004; Petzold et al., 2005; Perraudin et al., 2007; Shonija et al., 2007). The ability of soot PM to condense water also 
strongly influences its atmospheric lifetime. However, the details of the interaction of water with soot that determine its ability to nucleate liquid water and ice continue to be a primary atmospheric science research issue. The interaction of soot with water depends on the type of soot (lean vs. rich flame conditions) and changes with the degree of oxidative aging for undersaturated humidities (Chughtai et al., 1991; Alcala-Jornod and Rossi, 2004); this affects soot reactivity, since the presence of water on soot changes the accessibility of reactive surface sites and species. Similarly, the presence of adsorbed polycyclic aromatic hydrocarbons (PAHs) on soot affects it reactivity and depends strongly on the type of fuel combusted as well as on its combustion conditions (Jones et al., 2004).

\section{Recommendations for further work}

Future studies should address feedback mechanisms between soot type, oxidative aging, reactivity and hygroscopicity, as well as investigate multiphase processes in mixed particles composed of soot and other components. Furthermore, the impact of light induced processes and condensed phase radical chemistry has only recently been considered (Styler et al., 2009; Monge et al., 2010). (See Sect. 5.5 below)

Discussions of the wetting properties of soot and its associated ice and cloud nucleating properties often include the terms "hydrophilic" and "hydrophobic". These terms are qualitative in nature and have been coined to describe the results of contact angle measurements and thus refer to a bulk property of soot or other surfaces. It must be stressed that a molecular view on the wetting properties of submicron carbonaceous aerosol particles makes the use of these terms obsolete because even the most "hydrophobic" soot substrate shows molecular adsorption sites for $\mathrm{H}_{2} \mathrm{O}$ vapor (Alcala-Jornod et al., 2004). At the very least a molecular interpretation of the bulk hydrophobic/hydrophilic concept should be attempted.

A fundamental question about the interaction of free radicals such as $\mathrm{OH}, \mathrm{NO}_{2}$ and $\mathrm{NO}_{3}$, as well as other reactive atmospheric trace gases such as ozone, with PAHs adsorbed on soot concerns their relative reactivity with the carbonaceous substrate compared to adsorbed PAHs. Recent results, discussed above, suggest that the rate of reactive trace gas uptake by the substrate is three to four orders of magnitude faster than the rate of surface reaction of adsorbed PAHs. Additional experiments elucidating reactive branching ratios for adsorbed PAHs and relevant substrates are expected in the near future (Esteve et al., 2006; Perraudin et al., 2007; Miet et al., 2009a, b). Moreover, the underlying causes for the apparent non-reactivity of 30 to $70 \%$ of the PAHs adsorbed on laboratory soot with atmospheric trace gases such as $\mathrm{O}_{2}$ and $\mathrm{NO}_{2}$, except $\mathrm{OH}$, should be investigated. In contrast, $100 \%$ of PAHs adsorbed on $\mathrm{SiO}_{2}$ substrates are reactive. Furthermore, it is time to discover which organic surface func- tionalities make soot and other carbonaceous particles a good ice nucleus (IN) and/or condensation nucleus (CN) for water condensation.

\subsection{Trace gas uptake on solid and liquid organic surfaces}

The oxidation of organics in aerosol particles affects their physical and chemical properties. Laboratory and field measurements suggest that heterogeneous processes may play a central role in the atmospheric "aging" of organic particles. (Rudich 2003, 2007).

Ozone is efficiently removed by unsaturated (olefins, PAHs) organic surfaces as well as biological surfaces like leaves and lungs (de Gouw and Lovejoy, 1998; Moise and Rudich, 2000; Wadia et al., 2000; Pöschl et al., 2001; Moise and Rudich, 2002; Morris et al., 2002; Eliason et al., 2003; Katrib et al., 2003, 2004, 2005a, b; Broekhuizen et al., 2004; Kwamena et al., 2004, 2006; Mmereki et al., 2004; Thornberry and Abbatt, 2004; Donaldson et al., 2005; Moise et al., 2005; Ziemann, 2005; Knopf et al. 2005; Shiraiwa et al., 2009). In fact, reaction on surfaces occurs at a higher rate than in the gas phase (Wadia et al., 2000; Moise and Rudich, 2002; Donaldson et al., 2005; Donaldson and Vaida, 2006; Clifford et al., 2008; Gross and Bertram, 2008; Cape et al., 2009). The phase of the particle, as well as the matrix to which the organics attach, are important to the reaction rate and even small inhomogeneities can result in appreciable changes in the reaction rates (Moise and Rudich, 2000; Hearn and Smith, 2004; Kwamena et al., 2004; Hearn et al., 2005; Knopf et al., 2005; Kwamena et al., 2006; Griffiths et al., 2009; Sage et al., 2009). In some cases non-olefinic surfaces, such as organic monolayers, inhibit subsequent ozone uptake.

The $\mathrm{OH}$ radical reacts rapidly with all organic surfaces, often leading to fragmentation and release of oxygenated fragments to the gas phase (Bertram et al., 2001; Molina et al., 2004; Kwan et al., 2006; George et al., 2007; Vlasenko et al., 2008; Lambe et al., 2009). Release of gas phase products has also been observed in the reaction of ozone with organic surfaces (Thomas et al., 2001). The $\mathrm{OH}$ reaction proceeds via an Eley-Rideal direct abstraction mechanism. The high reactivity of the $\mathrm{OH}$ radical results in uptake coefficients close to unity, and probably does not depend on the relative humidity. Recently, reactivity of $\mathrm{OH}$ towards long chained alkanes was studied in a new method for measuring the heterogeneous chemistry of sub-micron organic aerosol particles (Che et al., 2009).

The $\mathrm{NO}_{3}$ radical reacts with organic surfaces, with uptake coefficient in the range $10^{-4}$ to $10^{-2}$ (Moise and Rudich, 2002; Docherty and Ziemann, 2006; Knopf et al., 2006; Gross and Bertram, 2008; Gross et al., 2009). Reactions with olefins are faster. The $\mathrm{NO}_{3}$ reaction does not lead to significant release of gas phase products (Knopf et al., 2006). It remains to be seen if $\mathrm{NO}_{3}$ uptake leads to formation of surface 
bound nitrates; similarly, organosulphates are observed following uptake of monoterpine oxides by acid sulfate particles (Iinuma et al., 2009).

Uptake of $\mathrm{N}_{2} \mathrm{O}_{5}$ by various organic surfaces has also been shown to depend on relative humidity, on particle phase, and on particle composition (Cosman and Bertram, 2008; Cosman et al., 2008; Griffiths et al., 2009). Field and laboratory studies have demonstrated that organic coatings on aqueous surfaces can decrease the reactive uptake of $\mathrm{N}_{2} \mathrm{O}_{5}$ (Brown et al., 2009); further details are presented in section 5.1.7 above.

Halogen atoms also react with all organic surfaces with a high uptake coefficient, also leading to release of fragments to the gas phase (Moise and Rudich, 2001).

Although polycyclic aromatic hydrocarbons (PAH) represent only a small mass fraction of the organic material associated with combustion related particulates they are of great concern due to their toxic and carcinogenic properties, especially after reacting with atmospheric oxidants. Aged PAHs consisting of singly or multiply substituted PAHs such as nitro- and hydroxyl-substituted PAHs as well as their corresponding quinones are usually encountered in the particulate phase because atmospheric reaction products generally have lower vapour pressures compared to reactant PAH and thus render these semivolatile hydrocarbons even less volatile.

Perraudin et al. (2007), Miet et al. (2009a, b) and others have investigated the reactions of $\mathrm{O}_{3}, \mathrm{NO}_{2}$ and $\mathrm{OH}$ with $\mathrm{PAH}$ adsorbed on various substrates such as graphite, diesel soot and silica $\left(\mathrm{SiO}_{2}\right)$ particles. On carbonaceous surfaces only a fraction of the adsorbed $\mathrm{PAH}$ reacts with the atmospheric oxidants in contrast to silica particles where $100 \%$ of the adsorbed PAH reacts. This begs the question of mass transport of small oxidant molecules and free radicals in nanoporous materials such as soot. Obviously mass transport is slow on the time scale of these experiments (several minutes), a fact also known from studies on bulk mineral dust samples discussed above (Sect. 5.2.2). The reactivity $\mathrm{OH}$ with $\mathrm{PAH}$ is independent of the $\mathrm{PAH}$ structure whereas $\mathrm{PAH} / \mathrm{NO}_{2}$ reactivity depends strongly on the identity of PAH. Similar to gas phase organic species $\mathrm{OH}$ reactions, the more exothermic reactions of $\mathrm{OH}$ radicals are less selective than the corresponding less exothermic reactions of the $\mathrm{NO}_{2}$ free radical. Heterogeneous reactions of $\mathrm{O}_{3}$ with particulate $\mathrm{PAH}$ were shown to be more rapid than those occurring in the gas phase, as opposed to reactions with $\mathrm{NO}_{2}$ and $\mathrm{OH}$ whose rates were faster in the gas compared to the particulate phase. In line with expectations, $\mathrm{OH}$ was approximately four orders of magnitude more reactive than $\mathrm{NO}_{2}$ towards surface-bound PAH. The comparison between carbonaceous and silica substrates leads to the conclusion that the $\mathrm{PAH} / \mathrm{NO}_{2}$ system depends on the nature of the substrate in contrast to $\mathrm{PAH} / \mathrm{O}_{3}$.

Gross and Bertram (2008) have examined the uptake of several atmospheric trace gases such as $\mathrm{NO}_{3}, \mathrm{~N}_{2} \mathrm{O}_{5}, \mathrm{NO}_{2}$, $\mathrm{HNO}_{3}$ and $\mathrm{O}_{3}$ on a variety of solid PAH surfaces in a coated wall laminar flow tube and come to the conclusion that only $\mathrm{NO}_{3}$ showed a significant uptake whereas all others showed immeasurably slow uptake kinetics. The initial uptake was very rapid $(\gamma>0.1)$ and settled down to a steady-state value after some tens of minutes on the order of several times $10^{-3}$ $(\gamma)$. This is in agreement with uptake experiments of $\mathrm{NO}_{3}$ on soot performed in a low-pressure flow reactor and suggests that PAH may be an important sink for $\mathrm{NO}_{3}$ under certain conditions (Karagulian and Rossi, 2007). It may be noted in passing that the initial uptake of $\mathrm{NO}_{3}$ by PAHs is as fast as it is on mineral dust surrogates (see section 5.2.2 and Karagulian and Rossi, 2005) in contrast to pure $\mathrm{H}_{2} \mathrm{O}$ ice (IUPAC, 2009; Crowley et al., 2010). Styler et al. (2008) observed that ozone uptake by solid pyrene films can be photoenhanced.

\section{Recommendations for further work}

Several issues remain unresolved and require further investigations. Surface-adsorbed water has been shown to affect reaction mechanisms and rates. Examples include reaction inhibition probably due to competition with the adsorbing reactant on adsorbing sites, or changes in reaction mechanisms due to still unidentified reactions of the water with intermediate species. Other outstanding issues are whether photoreductive uptake is a general phenomenon (see Sect. 5.5 below), and the degree to which the product of reactions are volatilized by the $\mathrm{OH}$ and halogen atom reactions, i.e. what are the VOC yields?

Another important issue to address is the chemical nature and hydrophobicity of the processed surfaces and the consequences of these transitions to aerosol microphysics and climatic impacts. Reactive uptake of ammonia by carboxylic acids can lead to changes in hygroscopic properties. However, the reactive uptake coefficients are currently unknown for this process (Dinar et al., 2008). We recommended that the effects of organic coatings on uptake by aerosols, the fate of the organic coating and the dependence on its origin (marine versus SOA) be more thoroughly investigated. Finally, studies of the role of complex organic films and their role in the heterogeneous chemistry of urban surfaces are highly desirable (Simpson et al., 2006).

\subsection{Photochemistry on atmospheric surfaces}

Organic aerosols have an atmospheric lifetime that can reach several weeks and it is therefore necessary to investigate their transformations in the atmospheric environment. Chemical aging may lead to changes both in the climatic and health effects of the aerosols due to variation in their composition, optical properties and hygroscopicity. In this context, many aging processes are still unknown; specifically photo-induced processes of atmospheric particles are definitely not well enough understood. Some of the fundamental unanswered questions are: 
Which photochemical processes occur in the atmosphere and how do they modify the optically absorbing organic component of the aerosols?

Can those processes constitute an alternative pathway for oligomer formation?

How can these processes affect the photo-oxidant budget?

Recent findings confirm the presence of light absorbing organic material (HULIS, biomass burning particles, PAHs) in atmospheric aerosols. Laboratory studies have shown the formation of oligomeric material with molecular mass ranging from 400-1000 Daltons within a few hours (Jang et al., 2002; Kalberer and Paulsen, 2004). The process occurs by classical carbonyl chemistry and by the action of radicals on the condensed precursors (Gelencser et al., 2003; Jang et al., 2002; Kalberer et al., 2004). During oxidative aging, initially non-absorbing organic compounds can be converted into compounds that significantly absorb UV and even visible light. This has been shown for hydroxy substituted aromatics typically associated with biomass burning aerosol (Gelencser et al., 2003; Krivacsy et al., 2000). A second type of coloured organic compounds is associated with soil dust, where humic substances of terrestrial origin are likely the major organic fraction (Miller et al., 2004). Coloured organic material is also produced by combustion processes, like biomass burning or fossil fuel combustion (GonzalezPerez et al., 2004).

The presence of light absorbing material in aerosol particles can allow photosensitized processes. A significant body of literature exists on photo-induced charge or energy transfer in organic molecules related to biochemistry and water waste treatment (Canonica et al., 2000, 2005; Giese et al., 2005). The primary absorbing species (photosensitizer) is excited into a triplet state and depending on the redox properties of available reaction partners and the medium, charge or energy transfer can occur to other molecules. Typically, photosensitized processes can lead to both reduction and oxidation of intermediates and final products. While aquatic photochemists have recognized a number of such processes that accelerate degradation of dissolved organic matter, (e.g. Haag et al., 1984; Zafiriou et al., 1984), little is currently known about photochemistry in atmospheric particles. Nevertheless, in view of the presence of partially oxidized organics in atmospheric particles, it has to be assumed that such processes will significantly affect their chemical properties during aging (Gomez et al., 2006; Park et al., 2006).

In particular, the presence of light has recently been shown to modify the reactivity at the gas-liquid interface. For example, George et al., 2005) showed that the uptake kinetics of $\mathrm{NO}_{2}$ on various surfaces, taken as proxies for organic surfaces encountered in the troposphere, was strongly enhanced once these surfaces were irradiated with light in the range $300-500 \mathrm{~nm}$, suggesting a mechanism in which photosensitized electron transfer is occurring. Stemmler et al. (2006) observed an even stronger photoenhancement on humic acid interfaces, on which the light-activated reduction of $\mathrm{NO}_{2}$ was observed to be a major source of gaseous HONO. These photoenhanced uptake kinetics were observed for both macroscopic plane surfaces and for submicron aerosols Stemmler et al., 2007) and also on PAH Brigante et al., 2008).

Similar observations were made for ozone reacting at organic interfaces made of humic acids D'Anna et al., 2009) or model compounds (such as benzophenone and phenol) under simulated atmospheric conditions with respect to relative humidity, pressure, temperature and $\mathrm{O}_{3}$ concentration using a coated flow tube reactor (Jammoul et al., 2008). Interestingly, the contact angle between these surfaces and water was observed to increase indicating higher organic film hydrophobicity upon combined exposure to light and ozone Nieto-Gligorovski et al., 2008). Not only are these interfaces more hydrophobic upon aging, they exhibit a new absorption band extending to $450 \mathrm{~nm}$. Appearance (or redshifting) of UV-visible features during SOA formation and aging were also observed during the processing of particles formed by the oxidation of limonene, indicating solar radiation may play a significant role in the processing of SOA in the atmosphere (Walser et al., 2007, 2008). Light absorbing oligomers were also observed from the aldol condensation of carbonyl in sulfuric acid solutions (Nozière and Esteve, 2005, 2007). Amino acid- and ammonium sulfate-catalyzed reactions in water and ionic solutions were also suggested to produce light-absorbing "humic-like" compounds (Nozière et al., 2007).

\section{Recommendations for further work}

The impact of light on atmospheric gas phase chemistry has been studied for a long time, especially its ability to break bonds (i.e., photolysis). Recently, it has been recognized that HULIS, a term used to describe high molecular weight, partially chromophoric organic material with some similarity to humic soil components, constitute an important fraction of organic matter in tropospheric aerosols. Therefore, it may be interesting to consider the impact of light on the chemical aging of organic aerosol particles. While the existence of HULIS is now certain, its atmospheric chemistry is still mostly unexplored. In this context, some of the fundamental unanswered questions are:

Which photochemical processes occur in the atmosphere and how do they modify the optically absorbing organic component of the aerosols?

Can those processes constitute an alternative pathway for oligomer formation?

How can these processes affect the photo-oxidant budget?

What is the role of adsorbed water on these chemical transformations? 


\section{Conclusions and outlook}

\subsection{Next steps}

Despite a large number of heterogeneous process studies over the past decades, which have improved our understanding of stratospheric ozone depletion, acid deposition, and aerosol PM chemistry, many heterogeneous processes fundamental to atmospheric chemistry are still poorly understood or need to be more fully characterized. Current knowledge is not sufficient to furnish a scientifically sound basis for constructing the chemical and physico-chemical process modules that need to be included in the improved numerical models of our atmospheric environment. Without further progress our ability to better assess the current and future states of our atmosphere and the life it supports will remain impaired.

Accordingly, many important questions concerning the fate (including uptake and accommodation processes) of numerous chemicals still remain to be answered. Sustained efforts will be required to:

Build evaluated databases for reaction rates and photochemical parameters for processes in the gas phase, on particle surfaces, and in the condensed phase

Implement theoretical methods as additional tools for predicting heterogeneous processes affecting degradation schemes, reaction rates, secondary product yields, and the aerosol formation potential of new chemicals under atmospheric conditions.

Improve Chemistry and Transport Models (CTMs) by implementing the newly gained knowledge in their chemical modules, thereby enhancing their usefulness as chemical assessment tools for policy makers with regard to the fate of new chemicals in the atmosphere.

Use the new kinetic parameters as they become available from the leading atmospheric chemistry laboratories in Europe and elsewhere to develop and apply multiphase modelling tools to interpret the results of large field measurement campaigns, thus maximising the amount of information, which may be obtained from these expensive activities.

Laboratory and supporting theoretical and computer simulation studies are essential in this respect as they are the unique way of providing scientifically sound and evaluated data for numerical atmospheric models.

\subsection{Evaluation and dissemination of trace gas uptake data}

We now consider how the knowledge gained on the subject of trace gas uptake in the atmosphere is transferred to the atmospheric community. The rate of a heterogeneous process is usually expressed in terms of the uptake coefficient, $\gamma$, which is defined as the fraction of molecules colliding with the surface in unit time that are permanently lost from the gas phase, e.g. by reaction on the surface or the bulk. A gas may also be taken up into a liquid due to its solubility. This is expressed in terms of the Henry's law constant, $\mathrm{H}\left(\mathrm{M} \mathrm{atm}^{-1}\right)$, which relates the partial pressure of the trace gas and its molar concentration in the condensed phase at equilibrium. In reactions on solid or liquid surfaces the equivalent measure of phase partitioning is the Langmuir equilibrium constant for surface adsorption. Under some conditions the uptake flux is also determined by diffusive transport in either the gas or condensed phase, requiring values for diffusion coefficients in either phase. The rate constants of condensed phase reactions may also determine uptake rates. Timely evaluation of the experimental data to provide optimum values of all these parameters and their open access presentation are essential requisites for advancement of this aspect of atmospheric chemistry.

The NASA Kinetics Panel and IUPAC Atmospheric Kinetics Evaluation Group have both addressed reviewed and evaluated kinetic parameters for heterogeneous reactions for use in atmospheric studies (NASA, 2006; IUPAC, 2009; Crowley et al., 2010). Relatively few reactions are sufficiently well defined to make recommendations for uptake coefficients. Part of the difficulty is the definition of the surface characteristics of the atmospheric particles supporting the reactions. Most progress has been made in understanding of the reactions at low temperature on supercooled liquid sulfate aerosols.

In their recent evaluation, the NASA panel (NASA, 2006) has presented recommendations for a full parameterization of the reactions of $\mathrm{N}_{2} \mathrm{O}_{5}, \mathrm{ClONO}_{2}$ and $\mathrm{HOCl}$ on super-cooled sulphuric acid aerosols for stratospheric conditions. This is based on the parameters $a, H, D_{g}, D_{l}, k_{\mathrm{rxn}}$ which give the overall uptake coefficient $\gamma$ in the resistance model. The resistance model is a useful approximation that is usually sufficiently accurate, but the same parameters are required for calculations with dynamic flux models. The NASA Panel has also evaluated uptake coefficients for selected heterogeneous reactions on a range of substrates of stratospheric relevance, including ice, NAT and other solid acid hydrates, liquid water, alumina and soot. They have also assembled an extended compilation of Henry's law parameters for pure water, and a procedure for estimating the effective Henry's law parameters for aqueous salt solutions. Updated NASA panel evaluations for gas uptake by aqueous liquid, ice, and alumina surfaces are scheduled for publication in late 2010.

Uptake on solids is in principle similar to uptake on liquids except that the diffusion into the condensed phase is usually negligible. Consequently, uptake on solids is prone to saturation phenomena leading to time dependent uptake kinetics requiring adsorption equilibrium constants and surface rate constants. Very few atmospheric models currently give a detailed explicit representation of these heterogeneous rate processes.

The IUPAC group assembled a large compilation of experimental data related to heterogeneous reactions on a range of substrates of atmospheric interest with relevance for both 
troposphere and stratosphere (IUPAC, 2009; Crowley et al., 2010). This group has recently extended their evaluations to include recommended parameters to describe partition coefficients and reactive uptake coefficients to solid surfaces. The new evaluation covers the heterogeneous processes on surfaces of: ice, mineral dust, sulfuric acid hydrate and nitric acid hydrate surfaces. Recommendations for uptake coefficients and adsorption parameters and are now presented on the IUPAC website. The material consists of an introduction to atmospheric heterogeneous processes, summary sheets containing the recommended uptake parameters for the evaluated processes, and four appendices containing the data sheets that provide information upon which the recommendations are made.

The review and evaluation of kinetic and thermodynamic data relevant to atmospheric processes is a tedious and sometimes thankless task that is performed on a volunteer basis by the NASA panel and IUPAC group members. However, it is a vital activity that confirms what we know and highlights what we do not know about important kinetic processes affecting atmospheric PM properties and trace gas chemistry.

\subsection{Support for ongoing trace gas uptake research}

Unfortunately, current European funding schemes do not provide strong support for such fundamental studies (but rather support field campaign and large scale modelling). This situation may be due to the fact that funding agencies are focusing on "simple" issues (such as ozone modelling) for which current state-of-art models may be an appropriate answer. However, do models really reflect current knowledge as made available by laboratory based studies? How confident are we that we have adequate knowledge of all relevant physico-chemical processes? These questions underline the need to strengthen the link between modellers and "lab scientists".

There have been a limited number of European programs that have supported the required laboratory studies. One of those is an ESF Research Networking Programme focused on "Interdisciplinary Tropospheric Research: From the Laboratory to Global Change (INTROP)", which has promoted exchange of knowledge and sharpening of common awareness through support for the organization of conferences and topical workshops. It has also promoted training of young scientists. Training of young scientists is a key feature for reaching and perpetuating the highest possible level of scientific research. Similar promotion of exchange and collaboration between atmospheric scientists, including those engaged in experimental studies in laboratories and simulation chamber facilities has been provided in ACCENT, Atmospheric Composition Change; the European Network of Excellence. Unfortunately, neither INTROP nor ACCENT can directly support the needed experimental studies. Both these programs came to an end in 2009 and follow-on programs are badly needed.
In North America funding for laboratory and supporting theoretical/simulation studies has either declined substantially or threatens to do so.

In the US redirection of NASA to focus on space exploration activities and technologies greatly reduced funding for Earth science topics, with core laboratory and theoretical science that is not directly related to analysis of satellite data suffering large cuts. There is currently an effort to better balance NASA's science and space exploration portfolios, but whether support for fundamental laboratory and supporting theoretical research will rebound is unclear. Prior research budget cuts at the Environmental Protection Agency have limited it as a funding source for topics relevant to this review; its research budget is currently increasing but these increases may be difficult to sustain. The Department of Energy's Atmospheric Science Program has recently been consolidated into a larger Atmospheric Systems Research program dominated by field measurements and atmospheric modelling; the future of its modest laboratory program is undefined. Atmospheric chemistry programs at the National Oceanic and Atmospheric Administration have also suffered budget reductions from historic highs. The Atmospheric Chemistry Program budget at the National Science Foundation has enjoyed modest growth, but is highly oversubscribed due to budget cuts at other US agencies.

In Canada, most current funding sources are highly targeted and do not provide funds for basic laboratory research in atmospheric processes. Two exceptions are the NSERC Discovery Grant program that provides relatively modest grants and the Foundation for Climate and Atmospheric Sciences (CFCAS) that has been the major source of funding for relevant laboratory and supporting theoretical work since 2000; CFCAS funding is scheduled to expire in 2010.

Without a reinvigorated commitment to fund fundamental laboratory and supporting theoretical/simulation work progress in meeting the scientific challenges discussed in this document will stall. As a result our ability to use atmospheric models to understand how our current atmosphere works and how it will behave in the future will fall well short of the level needed to assess what we need to do to provide life supporting air quality and a sustainable climate for coming generations.

Acknowledgements. We gratefully acknowledge financial support from the European Commission through the ACCENT (Atmospheric Composition Change - Network of Excellence) under Contract No. 505337. This support made possible the international gathering of scientists that led to this review. We also thank Hannah Barjat and Brianne Hills who helped with document preparation.

Edited by: V. F. McNeill 


\section{References}

Abbatt, J. P. D.: Heterogeneous interactions of $\mathrm{BrO}$ and $\mathrm{ClO}$ : Evidence for $\mathrm{BrO}$ surface recombination and reaction with $\mathrm{HSO}_{3}^{-} / \mathrm{SO}_{3}^{2}$, Geophys. Res. Lett., 23, 1681-1684, 1996.

Abbatt, J. P. D.: Interactions of atmospheric trace gases with ice surfaces: adsorption and reaction, Chem. Rev., 103, 4783-4800, 2003.

Abbatt, J. P. D. and Waschewsky, G. C. G.: Heterogeneous interactions of $\mathrm{HOBr}, \mathrm{HNO}_{3}, \mathrm{O}_{3}$ and $\mathrm{NO}_{2}$ with deliquescent $\mathrm{NaCl}$ aerosols at room temperature, J. Phys. Chem. A., 102, 37193725, 1998.

Adams, J. W., Holmes, N. S., and Crowley, J. N.: Uptake and reaction of $\mathrm{HOBr}$ on frozen and dry $\mathrm{NaCl} / \mathrm{NaBr}$ surfaces between 253 and 233 K, Atmos. Chem. Phys., 2, 79-91, doi:10.5194/acp2-79-2002, 2002.

Adams, J. W., Rodriguez, D., and Cox, R. A.: The uptake of $\mathrm{SO}_{2}$ on Saharan dust: a flow tube study, Atmos. Chem. Phys., 5, 26432676, doi:10.5194/acp-5-2643-2005, 2005.

Adamson, A. W. and Gast, A. P.: Physical chemistry of surfaces, J. Wiley and Sons, New York, NY, USA, 75-86, 1997.

Aguzzi, A. and Rossi, M. J.: The Kinetics of the uptake of $\mathrm{HNO}_{3}$ on Ice, Solid $\mathrm{H}_{2} \mathrm{SO}_{4} / \mathrm{H}_{2} \mathrm{O}$ and Solid Ternary Solutions of $\mathrm{H}_{2} \mathrm{SO}_{4} / \mathrm{HNO}_{3} / \mathrm{H}_{2} \mathrm{O}$ in the Temperature Range 180 to $211 \mathrm{~K}$, Phys. Chem. Chem. Phys., 3, 3707-3716, 2001.

Aguzzi, A., Flückiger, B. and Rossi, M. J.: The nature of the interface and the diffusion coefficient of $\mathrm{HCl} /$ ice and $\mathrm{HBr}$ /ice in the temperature range 190-205 K, Phys. Chem. Chem. Phys., 5, 4157-4169, 2003.

Alcala-Jornod, C. and Rossi, M. J.: Chemical kinetics of the interaction of $\mathrm{H}_{2} \mathrm{O}$ vapor with soot in the range $190=\mathrm{T} / \mathrm{K}=300$ : A diffusion tube study, J. Phys. Chem. A, 108, 10667-10680, 2004.

Alejandre, J., Tildesley, D. J., and Chapela, G. A.: MolecularDynamics Simulation of the Orthobaric Densities and SurfaceTension of Water, J. Chem. Phys., 102, 4574-4583, 1995.

Allen, H. C., Gragson, D. E., and Richmond, G. L.: Molecular structure and adsorption of dimethyl sulfoxide at the surface of aqueous solutions, J. Phys. Chem. B, 103, 660-666, 1999.

Allen, M. P., and Tildesley, D. J.: Computer Simulation of Liquids, Oxford University Press, New York, USA, 24-32, 1987.

Ammann, M. and Pöschl, U.: Kinetic model framework for aerosol and cloud surface chemistry and gas-particle interactions - Part 2: Exemplary practical applications and numerical simulations, Atmos. Chem. Phys., 7, 6025-6045, doi:10.5194/acp-7-60252007, 2007.

Ammann, M., Pöschl, U., and Rudich, Y.: Effects of reversible adsorption and Langmuir-Hinshelwood surface reactions on gas uptake by atmospheric particles, Phys. Chem. Chem. Phys., 5, 351-356, 2003.

Anonymous Referee \#1: Interactive comment on "Alkene ozonolysis SOA: inferences of composition and droplet growth kinetics from Köhler theory analysis" by A. Asa-Awuku et al., Anonymous Referee \#1, Atmos. Chem. Phys. Discuss., 7, S4052S4062, doi:10.5194/acp-7-S4052-2007, 2007.

Anttila, T., Kiendler-Scharr, A., Tillmann, R., and Mentel, T. F.: On the Reactive Uptake of Gaseous Compounds by OrganicCoated Aqueous Aerosols: Theoretical Analysis and Application to the Heterogeneous Hydrolysis of $\mathrm{N}_{2} \mathrm{O}_{5}$, J. Phys. Chem. A., 110, 10435-10443, 2006.

Archuleta, C. M., DeMott, P. J., and Kreidenweis, S. M.: Ice nu- cleation by surrogates for atmospheric mineral dust and mineral dust/sulfate particles at cirrus temperatures, Atmos. Chem. Phys., 5, 2617-2634, doi:10.5194/acp-5-2617-2005, 2005.

Ardura, D. and Donaldson, D. J.: Where does acid hydrolysis take place?, Phys. Chem. Chem. Phys., 11, 857-863, 2009.

Arens, F., Gutzwiller, L., Baltensperger, U., Heinz W. Gäggeler, H. W., and Ammann, M.: Heterogeneous reaction of $\mathrm{NO}_{2}$ on diesel soot particles, Environ. Sci. Technol., 35, 2191-2199, 2001.

Asa-Awuku, A., Engelhart, G. J., Lee, B. H., Pandis, S. N. and Nenes, A.: Relating CCN activity, volatility, and droplet growth kinetics of $\beta$-caryophyllene secondary organic aerosol, Atmos. Chem. Phys., 9, 795-812, doi:10.5194/acp-9-795-2009, 2009.

Asa-Awuku, A., Nenes, A., Gao, S., Flagan, R. C., and Seinfeld, J. H.: Water-soluble SOA from Alkene ozonolysis: composition and droplet activation kinetics inferences from analysis of CCN activity, Atmos. Chem. Phys., 10, 1585-1597, doi:10.5194/acp10-1585-2010, 2010.

Aubin, D. G. and Abbatt, J. P. D.: Interaction of $\mathrm{NO}_{2}$ with Hydrocarbon Soot: Focus on HONO Yield, Surface Modification and Mechanism, J. Phys. Chem. A, 111, 6263-6273, 2007.

Ardura, D. and Donaldson, D.J..: Where does acid hydrolysis take place?, Phys. Chem. Chem. Phys., 11, 857-863, 2009.

Aumont, B., Madronich, S., Ammann, M., Kalberer, M., Baltensperger, U., Hauglustaine, D. and Brocheton, F.: On the $\mathrm{NO}_{2}$ + Soot Reaction in the Atmosphere, J. Geophys. Res., 104, 17291736, 1999.

Autrey, T., Brown, A. K., Camaioni, D. M., Dupuis, M., Foster, N. S., and Getty, A.: Thermochemistry of aqueous hydroxyl radical from advances in photoacoustic calorimetry and ab initio continuum solvation theory, J. Am. Chem. Soc., 126, 3680-3681, 2004.

Baldwin, A. C. and Golden, D. M.: Heterogeneous atmospheric reactions - Sulfuric acid aerosols as tropospheric sinks, Science, 206, 562-563, 1979.

Badger, C. L., George, I., Griffiths, P. T., Abbatt, J. P. D., and Cox, R. A.: Reaction uptake of $\mathrm{N}_{2} \mathrm{O}_{5}$ by aerosol particles containing humic acid and ammonium sulfate, J. Phys. Chem. A., 110, 6986-6994, 2006.

Bartels-Rausch, T., Huthwelker, T., Gäggeler, H. W., and Ammann, M.: Atmospheric Pressure Coated-Wall Flow-Tube Study of Acetone Adsorption on Ice, J. Phys. Chem. A, 109, 4531-4539, 2005.

Behnke, W., Krüger, H.-U., Scheer, V., and Zetsch, C.: Formation of atomic $\mathrm{Cl}$ from sea spray via photolysis of nitryl chloride - determination of the sticking coefficient of $\mathrm{N} 2 \mathrm{O} 5$ on $\mathrm{NaCl}$ aerosol, J. Aerosol Sci., 22, 609-612, 1991.

Ben-Naim, A. and Marcus, Y.: Solvation Thermodynamics of Nonionic Solutes, J. Chem. Phys., 81, 2016-2027, 1984.

Benjamin, I.: Theoretical-Study of Ion Solvation at the Water Liquid-Vapor Interface, J. Chem. Phys., 95, 3698-3709, 1991.

Benjamin, I.: Structure, thermodynamics, and dynamics of the liquid/vapor interface of water/dimethylsulfoxide mixtures, J. Chem. Phys., 110, 8070-8079, 1999.

Berendsen, H. J. C., Postma, J. P. M., van Gunsteren, W. F., and Hermans, J.: Interaction models for water in relation to protein hydration, in: Intermolecular Forces, edited by: Pullman, B., 24, Reidel, Dordrecht, The Netherlands, 331-342, 1981.

Berendsen, H. J. C., Grigera, J. R., and Straatsma, T. P.: The Missing Term in Effective Pair Potentials, J. Phys. Chem., 91, 6269- 
6271, 1987.

Bertram, A. K., Ivanov, A. V., Hunter, M., Molina, L. T., and Molina, M. J.: The reaction probability of $\mathrm{OH}$ on organic surfaces of tropospheric interest, J. Phys. Chem. A, 105, 9415-9421, 2001.

Bertram, T. H., Thornton, J. A., Riedel, T. P., Middlebrook, A. M., Bahreini, R., Bates, T. S., Quinn, P. K. and Coffman, D. J.: Direct observations of $\mathrm{N}_{2} \mathrm{O}_{5}$ reactivity on ambient aerosol particles, Geophys. Res. Lett., 36, L19803, doi:10.1029/2009GL040248, 2009.

Biermann, U. M., Crowley, J. N., Huthwelker, T., Moortgat, G. K., Crutzen, P. J., and Peter, T.: FTIR studies on lifetime prolongation of stratospheric ice particles due to NAT coating, Geophys. Res. Lett., 25, 3939-3942, 1998.

Bougiatioti, A., Fountoukis, C., Kalivitis, N., Pandis, S. N., Nenes, A., and Mihalopoulos, N.: Cloud condensation nuclei measurements in the marine boundary layer of the Eastern Mediterranean: CCN closure and droplet growth kinetics, Atmos. Chem. Phys., 9, 7053-7066, doi:10.5194/acp-9-7053-2009, 2009

Boy, M., Kulmala, M., Ruuskanen,T., Pihlatie, M., Reissell, A., Aalto, P. P., Keronen, P., Dal Maso, M., Hellen, H., Hakola, H., Janssen, R., Hanke, M., and Arnold, F.: Sulphuric acid closure and contribution to nucleation mode particle growth, Atmos. Chem. Phys., 5, 863-878, doi:10.5194/acp-5-863-2005, 2005.

Brigante, M., Cazoir, D., D’Anna, B., George, C., and Donaldson, D. J.: Photoenhanced uptake of NO2 by pyrene solid films, J. Phys. Chem. A, 112, 9503-9508, doi:10.1021/jp802324g, 2008.

Broekhuizen, K. E., Thornberry, T., Kumar, P. P., and Abbatt, J. P. D.: Formation of cloud condensation nuclei by oxidative processing: Unsaturated fatty acids, J. Geophys. Res.-Atmos., 109, D24206, doi:10.1029/2004JD005298, 2004.

Brown, R. L.: Tubular Flow Reactors with First Order Kinetics, J. Res. Natl. Bur. Stand., 83, 1-8, 1978.

Brown, S. S., Ryerson, T. B., Wollny, A. G., Brock, C. A., Peltier, R., Sullivan, A. P., Weber, R. J., Dube, W. P., Trainer, M., Meagher, J. F., Fehsenfeld, F. C., and Ravishankara, A. R.: Variability in nocturnal nitrogen oxide processing and its role in regional air quality, Science, 311, 67-70, 2006.

Brown, S. S., Dube, W. P., Fuchs, F., et al.: Reactive uptake coefficients for $\mathrm{N}_{2} \mathrm{O}_{5}$ determined from aircraft measurements during the Second Texas Air Quality Study: Comparison to current model parameterizations, J. Geophys. Res., 114, D00F10, doi:1029/2008JD011679, 2009.

Buajarern, J. Mitchem, L., and Reid, J. P.: Manipulation and Characterization of Aqueous Sodium Dodecyl Sulfate/Sodium Chloride Aerosol Particles, J. Phys. Chem. A, 111, 13038-13045, 2007.

Buch, V., Milet, A., Vácha, R., Jungwirth, P., and Devlin, J. P.: Water surface is acidic, Proc. Natl. Acad. Sci., , 104, 7342-7347, 2007.

Caloz, F., Fenter, F, Tabor, K. D., and Rossi, M. J.: Paper I: Design and construction of a Knudsen-cell reactor for the study of heterogeneous reactions over the temperature range 130-750 K: Performances and limitations, Rev. Sci. Instrum., 68, 3172-3179, 1997.

Canneaux, S., Soetens, J. C., Henon, E., and Bohr, F.: Accommodation of ethanol, acetone and benzaldehyde by the liquid-vapor interface of water: A molecular dynamics study, Chem. Phys., $327,512-517,2006$.
Canonica, S., Hellrung, B., and Wirz, J.: Oxidation of Phenols by Triplet Aromatic Ketones in Aqueous Solution, J. Phys. Chem. A, 104, 1226-1232, 2000.

Canonica, S., Kohn, T., Mac, M., Real, F. J., Wirz, J., and Von Gunten, U.: Photosensitizer Method to Determine Rate Constants for the Reaction of Carbonate Radical with Organic Compounds, Environ. Sci. Technol., 39, 9182-9188, 2005.

Cantrell, W., Shaw, G., Cass, G. R., Chowdhury, Z., Hughes, L. S., Prather, K. A., Guazzotti, S. A., and Coffee, K. R.: Closure between aerosol particles and cloud condensation nuclei at Kaashidhoo Climate Observatory, J. Geophys. Res., 106, 28711 28718, 2001.

Cape, J. N., Hamilton, R., and Heal, M. R.: Reactive uptake of ozone at simulated leaf surfaces: Implications for 'non-stomatal' ozone flux, Atmos. Environ., 43, 1116-1123, 2009.

Cappa, C. D., Smith, J. D., Drisdell, W. S., Saykally, R. J., and Cohen, R. C.: Interpreting the H/D isotope fractionation of liquid water during evaporation without condensation, J. Phys. Chem. C, 111, 7011-7020, 2007.

Cappa, C.D., Drisdell, W. S., Smith, J. D., Saykally, R. J., and Cohen, R. C.: Isotope Fractionation of Water during Evaporation without Condensation. J. Phys. Chem. B, 109, 24391-24400, 2005.

Carignano, M. A., Jacob, M. M., and Avila, E. E.: On the uptake of ammonia by the water/vapor interface, J. Phys. Chem. A, 112, 3676-3679, 2008.

Carravetta, V. and Clementi, E.: Water Water Interaction Potential - An Approximation Of The Electron Correlation Contribution By A Functional Of The Scf Density-Matrix, J. Chem. Phys., 81, 2646-2651, 1984.

Carslaw, K. S., and Peter, T.: Uncertainties in reactive uptake coefficients for solid stratospheric particles.1. Surface Chemistry, Geophys. Res. Lett., 24, 1743-1746, 1997.

Castro, A., Bhattacharyya, K., and Eisenthal, K. B.: Energetics Of Adsorption Of Neutral And Charged Molecules At The AirWater-Interface By 2nd Harmonic-Generation - Hydrophobic And Solvation Effects, J. Chem. Phys., 95, 1310-1315, 1991.

Chandler, D.: Statistical-mechanics of isomerization dynamics in liquids and transition-state approximation, J. Chem. Phys., 68, 2959-2970, 1978.

Che, D. L., Smith, J. D., Leone, S. R., Ahmed, M., and Wilson, K. R.: Quantifying the reactive uptake of $\mathrm{OH}$ by organic aerosols in a continuous flow stirred tank reactor, Phys. Chem. Chem. Phys., 11, 7885-7895, 2009.

Chuang, P. Y.: Measurement of the timescale of hygroscopic growth for atmospheric aerosols, J. Geophys. Res.-Atmos., 108, 4282, doi:10.1029/2002JD002757, 2003.

Chughtai, A. R., Jassim, J. A., Peterson, J. H., Stedman, D. H., and Smith, D. M.: Spectroscopic And Solubility Characteristics Of Oxidized Soots, Aerosol Sci. Technol., 15, 112-126, 1991.

Clegg, S. L., Seinfeld, J. H., and Brimblecombe, P.: Thermodynamic modelling of aqueous aerosols containing electrolytes and dissolved organic compounds, J. Aerosol. Sci., 32, 713-738, 2001.

Clegg, S. M. and Abbatt, J. P. D.: Uptake of gas-phase $\mathrm{SO}_{2}$ and $\mathrm{H}_{2} \mathrm{O}_{2}$ by ice surfaces: dependence on partial pressure, temperature and surface acidity, J. Phys. Chem. A 105, 6630-6636, 2001

Clement, C. F., Kulmala, M., and Vesala, T.: Theoretical consideration on sticking probabilities, J. Aerosol Sci., 27, 869-882, 
1996.

Cilfford, D., Bartesis-Rausch, T. and Donaldson, D. J.: Suppression of aqueous surface hydrolysis by monolayers of short chain organic amphiphiles, Phys. Chem. Chem. Phys., 9, 1362-1369, 2007.

Clifford, D. and Donaldson, D. J.: Direct experimental evidence for a heterogeneous reaction of ozone with bromide at the airaqueous interface, J. Phys. Chem. A, 111, 9809-9814, 2007.

Clifford, D., D. J. Donaldson, D. J., Brigante, M., D'Anna, B., and George, C.: Reactive uptake of ozone by chlorophyll at aqueous surfaces, Environ. Sci. Technol., 42, 1138-1143, 2008.

Cooper, P. and Abbatt, J. P. D.: Heterogeneous Interactions of $\mathrm{OH}$ and $\mathrm{HO}_{2}$ Radicals with Surfaces Characteristic of Atmospheric Particulate Matter, J. Phys. Chem., 100, 2249-2254, 1996.

Cosman, L. M. and Bertram, A. K.: Reactive uptake of N2O5 on aqueous $\mathrm{H} 2 \mathrm{SO} 4$ solutions coated with 1-component and 2component monolayers, J. Phys. Chem. A, 112, 4625-4635, 2008.

Cosman, L. M., Knopf, D. A., and Bertram, A. K.: N2O5 reactive uptake on aqueous sulfuric acid solutions coated with branched and straight-chain insoluble organic surfactants, J. Phys. Chem. A, 112, 2386-2396, 2008.

Crowley, J. N., Ammann, M., Cox, R. A., Hynes, R. G., Jenkin, Me. E., Mellouki, A., Rossi, M. J., Troe, J., and Wallington, T. J.: Evaluated kinetic and photochemical data for atmospheric chemistry: Volume V - heterogeneous reactions on solid substrates, Atmos. Chem. Phys., 10, 9059-9223, doi:10.5194/acp-10-90592010, 2010.

Cwiertny, D. M., Young, M. A., and Grassian, V. H.: Chemistry and photochemistry of mineral dust aerosol, Ann. Rev. Phys. Chem., 59, 27-51, 2008.

D’Anna, B., Jammoul, A., George, C., Stemmler, K., Fahrni, S., Ammann, M., and Wisthaler, A.: Light-induced ozone depletion by humic acid films and submicron aerosol particles, J. Geophys. Res.-Atmos., 114, 12, D12301, doi:10.1029/2008jd011237, 2009.

Dabkowski, J., Zagorska, I., Dabkowska, M., Koczorowski, Z., and Trasatti, S.: Adsorption of DMSO at the free surface of water: Surface excesses and surface potential shifts in the low concentration range, J. Chem. Soc.-Faraday Trans., 92, 3873-3878, 1996.

Dang, L. X. and Chang, T. M.: Molecular dynamics study of water clusters, liquid, and liquid-vapor interface of water with manybody potentials, J. Chem. Phys., 106, 8149-8159, 1997.

Dang, L. X. and Feller, D.: Molecular dynamics study of waterbenzene interactions at the liquid/vapor interface of water, J. Phys. Chem. B, 104, 4403-4407, 2000.

Dang, L. X. and Garrett, B. C.: Molecular mechanism of water and ammonia uptake by the liquid/vapor interface of water, Chem. Phys. Lett., 385, 309-313, 2004.

Danckwerts, P. V.: Gas-Liquid Reactions, McGraw-Hill, New York, NY, USA, 276 pp., 1970.

Davidovits, P., Hu, J. H., Worsnop, D. R., Zahniser, M. S., and Kolb, C. E.: Entry of Gas Molecules into Liquids, Faraday Discussions, 100, 65-82, 1995.

Davidovits, P., Worsnop, D. R., Williams, L. R., Kolb, C. E., and Gershenzon, M.: Comment on "Mass accommodation coefficient of water: Molecular dynamics simulation and revised analysis of droplet train/flow reactor experiment", J. Phys. Chem. B,
109, 14742-14746, 2005.

Davidovits, P., Worsnop, D. R., Jayne, J. T., Kolb, C. E., Winkler, P., Vrtala, A., Wagner, P. E., Kulmala, M., Lehtinen, K. E. J., Vesala, T. and Mozurkewich, M.: Mass accommodation coefficient of water vapor on liquid water, Geophys. Res. Lett. 31, L22111, doi:10.1029/2004GL020835, 2004.

Davidovits, P., Kolb, C. E., Williams, L. R., Jayne, J. T., and Worsnop, D. R.: Mass Accommodation and Chemical Reactions at Gas-Liquid Interfaces, Chem. Rev., 106, 1323-1354, doi:10.1021/cr040366k, 2006.

Davies, J. A. and Cox, R. A..: Kinetics of the Heterogeneous Reaction of $\mathrm{HNO}_{3}$ with $\mathrm{NaCl}$ : Effect of Water Vapor, J. Phys. Chem. A, 102, 7631-7642, 1998.

Davis, E. J.: A History and State-of-the-Art of Accommodation Coefficients, Atmos. Res., 82, 561-578, 2006.

Davis, E. J.: Interpretation of Uptake Coefficient Data Obtained with Flow Tubes, J. Phys. Chem. A, 112, 1922-1932, 2008.

Decesari, S., Facchini, M. C., Matta, E., Mircea, M., Fuzzi, S., Chughtai, A. R., and Smith, D. M..: Water soluble organic compounds formed by oxidation of soot, Atmos. Environ., 36, 18271832, 2002.

de Gouw, J. A. and Lovejoy, E. R.: Reactive uptake of ozone by liquid organic compounds, Geophys. Res. Lett., 25, 931-934, 1998.

Delval, C., Flückiger, B., and Rossi, M. J.: The rate of water vapor evaporation from ice substrates in the presence of $\mathrm{HCl}$ and $\mathrm{HBr}$ : implications for the lifetime of atmospheric ice particles, Atmos. Chem. Phys. , 3, 1131-1145, 2003.

Delval, C. and Rossi, M. J.: The kinetics of condensation and evaporation of $\mathrm{H}_{2} \mathrm{O}$ from pure ice in the range 173-223 K: A quartz crystal microbalance study, Phys. Chem. Chem. Phys., 6, 46654676, 2004.

Demou, E. and Donaldson, D. J.: Adsorption of atmospheric gases at the air-water interface. 4: The influence of salts, J. Phys. Chem. A, 106, 982-987, 2002.

Dinar, E., Anttila, T., and Rudich, Y.: CCN activity and hygroscopic growth of organic aerosols following reactive uptake of ammonia, Environ. Sci. Technol. 42, 793-799, 2008.

Djikaev, Y. S. and Tabazadeh, A.: Effect of adsorption on the uptake of organic trace gas by cloud droplets, J. Geophys. Res.-Atmos., 108, 4689, doi:10.1029/2003JD003741, 2003.

Docherty, K. S. and Ziemann, P. J.: Reaction of oleic acid particles with $\mathrm{NO}_{3}$ radicals: Products, mechanism, and implications for radical-initiated organic aerosol oxidation, J. Phys. Chem. A, 110, 3567-3577, 2006.

Donaldson, D. J., Guest, J. A., and Goh, M. C.: Evidence for Adsorbed $\mathrm{SO}_{2}$ at the Aqueous Air Interface, J. Phys. Chem., 99, 9313-9315, 1995.

Donaldson, D. J.: Adsorption of atmospheric gases at the air-water interface. I. $\mathrm{NH}_{3}$, J. Phys. Chem. A, 103, 62-70, 1999.

Donaldson, D. J. and Anderson, D.: Adsorption of atmospheric gases at the air-water interface. 2. C-1-C-4 alcohols, acids, and acetone, J. Phys. Chem. A, 103, 871-876, 1999.

Donaldson, D. J., Mmereki, B. T., Chaudhuri, S. R., Handley, S., and Oh, M.: Uptake and reaction of atmospheric organic vapours on organic films, Faraday Discuss., 130, 227-239, 2005.

Donaldson, D. J. and Vaida, V.: The influence of organic films at the air-aqueous boundary on atmospheric processes, Chem. Rev., 106, 1445-1461, 2006.

Donaldson, D. J. and Valsaraj, K. T.: Adsorption and Reaction of 
Trace Gas-Phase Organic Compounds on Atmospheric Water Surfaces: A Critical Review, Environ. Sci. Technol., 44, 865873, 2010.

Drisdell, W. S., Cappa, C. D., Smith, J. D., Saykally, R. J., and Cohen, R. C.: Determination of the evaporation coefficient of D2O, Atmos. Chem. Phys., 8, 6699-6706, doi:10.5194/acp-86699-2008, 2008.

Eliason, T. L., Aloisio, S., Donaldson, D. J., Cziczo, D. J., and Vaida, V.: Processing of unsaturated organic acid films and aerosols by ozone, Atmos. Environ., 37, 2207-2219, 2003.

Engelhart, G. J., Asa-Awuku, A., Nenes, A., and Pandis, S. N.: $\mathrm{CCN}$ activity and droplet growth kinetics of fresh and aged monoterpene secondary organic aerosol, Atmos. Chem. Phys., 8, 3937-3949, doi:10.5194/acp-8-3937-2008, 2008.

Esteve, W., Budzinski, H., and Villenave, E. : Relative rate constants for the heterogeneous reactions of $\mathrm{NO}_{2}$ and $\mathrm{OH}$ radicals with polycyclic aromatic hydrocarbons adsorbed on carbonaceous particles. Part 2: PAHs adsorbed on diesel particulate exhaust SRM 1650a, Atmos. Environ., 40, 201-211, 2006.

Ewald, P. P.: The calculation of optical and electrostatic grid potential, Ann. Phys.-Berlin, 64, 253-287, 1921.

Falkovich, A. H. and Rudich, Y..: Analysis of semivolatile organic compounds in atmospheric aerosols by direct sample introduction thermal desorption GC/MS, Environ. Sci. Technol., 35, 2326-2333, 2001.

Falkovich, A. H., Schkolnik, G., Ganor, E., and Rudich, Y.: Adsorption of organic compounds pertinent to urban environments onto mineral dust particles, J. Geophys. Res., 109, D01201, doi:1029/2003JD003919, 2004.

Ferry, D., Suzanne, J., Nitsche, S., Popovitcheva, O. B., and Shonija, N. K.: Water adsorption and dynamics on kerosene soot under atmospheric conditions, J. Geophys. Res.-Atmos., 107(D23), doi:10.1029/2002JD002459, 2002.

Finlayson-Pitts, B. J.: The tropospheric chemistry of sea-salt: A molecular level view of the chemistry of $\mathrm{NaCl}$ and $\mathrm{NaBr}$, Chem. Rev., 103, 4801-4822, 2003.

Finlayson-Pitts, B. J. and Pitts Jr. J. N.: Chemistry of the Upper and Lower Atmosphere: Theory, Experiments, and Applications, Academic Press, San Diego, CA, USA, 969 pp., 1999.

Folkers, M., Mentel, T. H., and Wahner, A.: Influence of an organic coating on the reactivity of aqueous aerosols probed by the heterogeneous hydrolysis of $\mathrm{N}_{2} \mathrm{O}_{5}$, Geophys. Res. Lett., 30, 1644, doi:10.1029/2003GL017168, 2003.

Fountoukis, C. and Nenes, A.: Continued development of a cloud droplet formation parameterization for global climate models, J. Geophys. Res., 110, D11212, doi:10.1029/2004JD005591, 2005.

Fountoukis C., Nenes, A., Meskhidze, N., et al.: Aerosol-cloud drop concentration closure for clouds sampled during the International Consortium for Atmospheric Research on Transport and Transformation 2004 campaign, J. Geophys. Res., 112, D10S30, doi:10.1029/2006JD007272, 2007.

Fried, A., Henry, B. E., Calvert, J. G., and Mozurkewich, M.: The Reaction Probability of $\mathrm{N}_{2} \mathrm{O}_{5}$ with Sulfuric-Acid Aerosols at Stratospheric Temperatures and Compositions, J. Geophys. Res.Atmos., 99, 3517-3532, 1994.

Frinak, E. and Abbatt, J. P. D.: $\mathrm{Br}_{2}$ production from the heterogeneous reaction of gas-phase $\mathrm{OH}$ with aqueous salt solutions: Impacts of acidity, halide concentration and organic surfactants, J. Phys. Chem. A, 110, 10456-10464, 2006.
Frinak, E. K., Wermeille, S. J., Mashburn, C. D., Tolbert, M. A., and Pursell, C. J.: Heterogeneous reaction of gaseous nitric acid on $\gamma$-phase iron(III)oxide, J. Phys. Chem., 108, 1560-1566, 2004.

Fuchs, N. A. and Sutugin, A. G.: Highly Dispersed Aerosols, Ann Arbor Science Publishers, Ann Arbor, MI, USA, 105 pp., 1970.

Fuzzi, S., Andreae, M. O., Huebert, B. J., Kulmala, M., Bond, T. C., Boy, M., Doherty, S. J., Guenther, A., Kanakidou, M., Kawamura, K., Kerminen, V.-M., Lohmann, U., Russell, L. M., and Pöschl, U.: Critical Assessment of the Current State of Scientific Knowledge, Terminology, and Research Needs Concerning the Role of Organic Aerosols in the Atmosphere, Climate, and Global Change, Atmos. Chem. Phys., 6, 2017-2038, doi:10.5194/acp-6-2017-2006, 2006.

Gao, R. S., Popp, P. J., Fahey, D. W., Marcy, T. P., Herman, R. L., Weinstock, E. M., Baumgardner, D. G., Garrett, T. J., Rosenlof, K. H., Thompson, T. L., Bui, P. T., Ridley, B. A., Wofsy, S. C., Toon, O. B., Tolbert, M. A., Kärcher, B., Peter, T., Hudson, P. K., Weinheimer, A. J., and Heymsfield, A. J.: Evidence that nitric acid increases relative humidity in low-temperature Cirrus clouds, Science, 303, 516-520, 2004.

Garrett, B. C., Schenter, G. K., and Morita, A.: Molecular simulations of the transport of molecules across the liquid/vapor interface of water, Chem. Rev., 106, 1355-1374, 2006.

Gelencser, A., Hoffer, A., Kiss, G., Tombacz, E., Kurdi, R., and Bencze, L.: In-situ Formation of Light-Absorbing Organic Matter in Cloud Water, J. Atmos. Chem., 45, 25-33, $2003 \mathrm{a}$.

Gelencser, A., Hoffer, A., Kiss, G., Tombacz, E., Kurdi, R., and Bencze, L.: In-situ Formation of Light-Absorbing Organic Matter in Cloud Water, J. Atmos. Chem., 45, 25-33, 2003 b.

George, C., Ponche, J. L., Mirabel, P., Behnke, W., Scheer, V., and Zetzsch, C.: Study of the uptake of $\mathrm{N}_{2} \mathrm{O}_{5}$ by water and $\mathrm{NaCl}$ solutions, J. Phys. Chem., 98, 8780-8784, 1994.

George, C., Strekowski, R. S., Kleffmann, J., Stemmler, K., and Ammann, M.: Photoenhanced uptake of gaseous NO2 on solidorganic compounds: a photochemical source of HONO?, Faraday Discuss., 130, 195-210, doi:10.1039/b417888m, 2005.

George, I. J., Vlasenko, A., Slowik, J. G., Broekhuizen, K., and Abbatt, J. P. D.: Heterogeneous oxidation of saturated organic aerosols by hydroxyl radicals: Uptake kinetics, condensed-phase products, and particle size change, Atmos. Chem. Phys., 7, 4187-4201, doi:10.5194/acp-7-4187-2007, 2007.

George, I, J. Slowik, J. G., and Abbatt, J. P. D.: Chemical aging of ambient organic aerosol from heterogeneous reaction with hydroxyl radicals, Geophys. Res. Lett., 35, L13811, doi:10.1029/2008GL033884, 2008.

Ghosal, S., Hemminger, J. C., Bluhm, H., Mun, B. S., Hebenstreit, E. L. D., Ketteler, G., Ogletree, D. F., Requejo, F. G., and Salmeron, M.: Electron Spectroscopy of Aqueous Solution Interfaces Reveals Surface Enhancement of Halides, Science, 307, 563-566, 2005.

Giese, B., Napp, M., Jacques, O., Boudebous, H., Taylor, A. M., and Wirz, J.: Multistep electron transfer in oligopeptides: Direct observation of radical cation intermediates, Angew. Chem. Int. Ed., 44, 4073-4075, 2005.

Girardet, C. and Toubin, C.: Molecular atmospheric pollutant adsorption on ice: a theoretical survey, Surf. Sci. Rep., 44, 159238, 2001.

Golden, D. M., Spokes, G. N., and Benson, S. W.: Very Low Pressure Pyrolysis (VLPP); a versatile kinetic method, Angew. 
Chem., 85, 602-614, 1973.

Gomez, A. L., Park, J., Walser, M. L., Lin, A., and Nizkorodov, S. A.: UV photodissociation spectroscopy of oxidized undecylenic acid films, J. Phys. Chem. A, 110, 3584-3592, 2006.

Gonzalez-Perez, J. A., Gonzalez-Vila, F. J., Almendros, G., and Knicker, H.: The effect of fire on soil organic matter - a review, Environ. Int., 30, 855-870, 2004.

Goodman, A. I., Bernard, E. T., and Grassian, V. H.: Spectroscopic study of nitric acid and water adsorption on oxide particles: enhanced nitric acid uptake kinetics in the presence of adsorbed water, J. Phys. Chem. A 105, 6443-6457, 2001.

Gopalakrishnan, S., Liu, D. F., Allen, H. C., Kuo, M., and Shultz, M. J.: Vibrational spectroscopic studies of aqueous interfaces: Salts, acids, bases, and nanodrops, Chem. Rev., 106, 1155-1175, 2006.

Griffiths, P. T., Badger, C. L., Cox, R. A., Folkers, M., Henk, H. H., and Mentel, T. F.: Reactive Uptake of N2O5 by Aerosols Containing Dicarboxylic Acids. Effect of Particle Phase, Composition, and Nitrate Content, J. Phys. Chem. A, 113, 5082-5090, 2009.

Gross, S. and Bertram, A.: Reactive uptake of $\mathrm{NO}_{3}, \mathrm{~N}_{2} \mathrm{O}_{5}, \mathrm{NO}_{2}$, $\mathrm{HNO}_{3}$ and $\mathrm{O}_{3}$ on three types of polycyclic hydrocarbon surfaces, J. Phys. Chem. A, 112, 3104-3113, 2008.

Gross, S., R. Iannone, Song, X., and Bertram, A.: Reactive uptake studies of $\mathrm{NO}_{3}$ and $\mathrm{N}_{2} \mathrm{O}_{5}$ on alkenoic acid, alkanoate, and polyalcohol substrates to probe nighttime aerosol chemistry, Phys. Chem. Chem. Phys., 11, 7792-7803, 2009.

Grote, R. T. and Hynes, J. T.: G-H theory, J. Chem. Phys., 73, 27152732, 1980.

Guimbaud, C., Arens, F., Gutzwiller, L., Gaggeler, H. W., and Ammann, M.: Uptake of $\mathrm{HNO}_{3}$ to deliquescent sea-salt particles: a study using the short-lived radioactive isotope tracer N-13, Atmos. Chem. Phys., 2, 249-257, doi:10.5194/acp-2-249-2002, 2002

Gustafsson R. J., Orlov, A., Badger, C. L., Griffiths, P. T., Cox, R. A., and Lambert, R. M.: A comprehensive evaluation of water uptake on atmospherically relevant mineral surfaces: DRIFT spectroscopy, thermogravimetric analysis and aerosol growth measurements, Atmos. Chem. Phys., 5, 34153421, doi:10.5194/acp-5-3415-2005, 2005.

Gustafsson, R. J., Orlov, A., Griffiths, P. T., Cox, R. A., and Lambert, R. M.: Reduction of $\mathrm{NO}_{2}$ to nitrous acid on illuminated titanium dioxide aerosol surfaces: implications for photocatalysis and atmospheric chemistry, Chem. Comm., 37, 3936-3938, 2006.

Haag, W. R., Hoigne, J., Gassman, E., and Braun, A. M.: Singlet Oxygen in Surface Waters .2. Quantum Yields of Its Production by Some Natural Humic Materials as a Function of Wavelength, Chemosphere, 13, 641-650, 1984.

Hallquist, M., Stewart, D. J., Baker, J., and Cox, R. A.: Hydrolysis of $\mathrm{N}_{2} \mathrm{O}_{5}$ on sulphuric acid aerosols, J. Phys. Chem. A., 104, 3984-3990, 2000.

Hallquist, M., Stewart, D. J., Stephenson, S. K., and Cox, R. A.: Hydrolysis of N2O5 on sub-micron sulphate aerosols, Phys. Chem. Chem. Phys., 5, 3453-3463, 2003.

Hanisch, F. and Crowley, J. N.: Heterogeneous reactivity of gaseous nitric acid on $\mathrm{Al} 2 \mathrm{O} 3, \mathrm{CaCO} 3$ and atmospheric dust samples: A Knudsen cell study, J. Phys. Chem. A 105, 3096-3106, 2001a.

Hanisch, F. and Crowley, J. N.: The heterogeneous reactivity of gaseous nitric acid on authentic mineral dust samples, and on individual mineral and clay mineral components, Phys. Chem. Chem. Phys., 3, 2474-2482, 2001 b.

Hanisch, F. and Crowley, J. N.: Ozone: decomposition on Saharan dust: an experimental investigation, Atmos. Chem. Phys., 3, 119-130, 2003.

Hanson, D. and Mauersberger, K.: $\mathrm{HCl} / \mathrm{H}_{2} \mathrm{O}$ Solid Phase Vapor Pressures and $\mathrm{HCl}$ Solubility in Ice, J. Phys. Chem., 94, 47004705, 1990.

Hanson, D., Burkholder, J. B., Howard, C. J., and Ravishankara, A. R.: Measurement of $\mathrm{OH}$ and $\mathrm{HO}_{2}$ Radical Uptake Coefficients on Water and Sulfuric Acid Surfaces, J. Phys. Chem., 96, 49794985, 1992.

Hanson, D. R. and Lovejoy, E. R.: The reaction of $\mathrm{ClONO}_{2}$ with submicometer sulfuric acid aerosol, Science, 267, 1326-1328, 1995.

Hanson, D. R. and Lovejoy, E. R.: Heterogeneous reactions in liquid sulfuric acid: $\mathrm{HOCl}+\mathrm{HCl}$ as a model system, J. Phys. Chem., 100, 6397-6405, 1996.

Hanson, D. R. and Kosciuch, E.: Reply to "Comment on "The $\mathrm{NH}_{3}$ mass accommodation coefficient for uptake onto sulfuric acid solutions"," J. Phys. Chem. A., 108, 8549-8551, 2004.

Hanson, D. R.: Surface specific reactions on liquids, J. Phys. Chem. B., 101, 4998-5001, 1997a.

Hanson, D. R.: Reaction of $\mathrm{N}_{2} \mathrm{O}_{5}$ with $\mathrm{H}_{2} \mathrm{O}$ on bulk liquids and on particles and the effect of dissolved $\mathrm{HNO}_{3}$, Geophys, Res. Lett., 24, 1087-1090, 1997b.

Hanson, D. R.: Reaction of $\mathrm{ClONO}_{2}$ with $\mathrm{H}_{2} \mathrm{O}$ and $\mathrm{HCl}$ in sulfuric acid and $\mathrm{HNO}_{3} / \mathrm{H}_{2} \mathrm{SO}_{4} / \mathrm{H}_{2} \mathrm{O}$ mixtures, J. Phys. Chem. A., 102, 4794-4807, 1998.

Hartkopf, A. and Karger, B. L.: Study of Interfacial Properties of Water by Gas-Chromatography, Acc. Chem. Res., 6, 209-216, 1973.

Hearn, J. D. and Smith, G. D.: A chemical ionization mass spectrometry method for the online analysis of organic aerosols, Anal. Chem., 76, 2820-2826, 2004.

Hearn, J. D., Lovett, A. J., and Smith, G. D.: Ozonolysis of oleic acid particles: evidence for a surface reaction and secondary reactions involving Criegee intermediates, Phys. Chem. Chem. Phys., 7, 501-511, 2005.

Henson, B. F., Wilson, K. R., Robinson, J. M., Noble, C. A., Casson, J. L., and Worsnop, D. R.: Experimental isotherms of $\mathrm{HCl}$ on $\mathrm{H}_{2} \mathrm{O}$ ice under stratospheric conditions: connections between bulk and interfacial thermodynamics, J. Chem. Phys., 121, 84868499., 2004.

Hessberg von, P., Pouvesle, N., Winkler, A. K., Schuster, G., and Crowley, J. N.: Interaction of formic and acetic acid with ice surfaces between 187 and $227 \mathrm{~K}$. Investigation of single species- and competitive adsorption, Phys. Chem. Chem. Phys., 10, 23452355, 2008.

Hoff, J. T., Mackay, D., Gillham, R., and Shiu, W. Y.: Partitioning of Organic-Chemicals at the Air-Water-Interface in Environmental Systems, Environ. Sci. Technol., 27, 2174-2180, 1993.

Hu, J. H., Shi, Q., Davidovits, P., Worsnop, D. R., Zahniser, M. S., and Kolb, C. E.: Reactive Uptake of $\mathrm{Cl} 2(\mathrm{G})$ and $\mathrm{Br} 2(\mathrm{G})$ by Aqueous Surfaces as a Function of $\mathrm{Br}-$ and I- Ion Concentration - the Effect of Chemical-Reaction at the Interface, J. Phys. Chem., 99, 8768-8776, 1995.

Hu, J. H. and Abbatt, J. P. D.: Reaction Probabilities for $\mathrm{N}_{2} \mathrm{O}_{5}$ 
Hydrolysis on Sulfuric Acid and Ammonium Sulfate Aerosols at Room Temperature, J. Phys. Chem. A, 101, 871-878, 1997.

Huff, A. K. and Abbatt, J. P. D.: Gas-Phase $\mathrm{Br}_{2}$ Production in Heterogeneous Reactions of $\mathrm{Cl} 2, \mathrm{HOCl}$ and $\mathrm{BrCl}$ with Halide-Ice Surfaces, J. Phys. Chem. A., 104, 7284-7293, 2000.

Huff, A. K. and Abbatt, J. P. D.: Kinetics and Product Yields in the Heterogeneous Reactions of $\mathrm{HOBr}$ with Simulated Sea Ice Surfaces, J. Phys. Chem. A., 106, 5279-5287, 2002.

Hunt, S. W., Roeselová, M., Wang, W., Wingen, L. M., Knipping, E. M., Tobias, D. J., Dabdub, D., and Finlayson-Pitts, B. J.: Formation of Molecular Bromine from the Reaction of Ozone with Deliquesced $\mathrm{NaBr}$ Aerosol: Evidence for Interface Chemistry, J. Phys. Chem. A., 108, 11559-11572, 2004.

Huntzicker, J. J., Cary, R. A., and Ling, C. S.: Neutralization of Sulfuric Acid Aerosol by Ammonia, Environ. Sci. Technol., 14, 819-824, 1980.

Huthwelker, T., Ammann, M., and Peter, T.: The uptake of acidic gases on ice, Chem. Rev., 106, 1375-1444, 1980, 2006.

Hynes, J. T.: The theory of reactions in solution, in: Theory of Chemical Reaction Dynamics, edited by: Baer, M., CRC Press, Boca Raton, FL, USA, 171-234, 1985.

Iinuma, Y., Boge, O. Kahnt, A., et al.: Laboratory chamber studies on the formation of organosulfates from reactive uptake of monoterpene oxides, Phys. Chem. Chem. Phys., 11, 7985-7997, 2009.

Ismail, A. E., Grest, G. S., and Stevens, M. J.: Capillary waves at the liquid-vapor interface and the surface tension of water, J. Chem. Phys., 125, 014702, doi:10.1063/1.2209240, 2006.

IUPAC: IUPAC Subcommittee for Gas Kinetic Data Evaluation; http://www.iupac-kinetic.ch.cam.ac.uk/ International Union of Pure and Applied Chemistry, 2009.

Jammoul, A., Gligorovski, S., George, C., and D'Anna, B.: Photosensitized heterogeneous chemistry of ozone on organic films, J. Phys. Chem. A, 112, 1268-1276, doi:10.1021/jp074348t, 2008.

Jang, M., Czoschke, N. M., Lee, S., and Kamens, R. M.: Heterogeneous Atmospheric Aerosol Production by Acid-Catalyzed Particle-Phase Reactions, Science, 298, 814-817, 2002.

Jayne, J. T., Davidovits, P., Worsnop, D. R., Zahniser, M. S., and Kolb, C. E.: Uptake of $\mathrm{SO}_{2}(\mathrm{~g})$ by aqueous surfaces as a function of PH - The effect of chemical-reaction at the interface, J. Phys. Chem., 94, 6041-6048, 1990.

Jayne, J. T., Duan, S. X., Davidovits, P., Worsnop, D. R., Zahniser, M. S., and Kolb, C. E.: Uptake of Gas-Phase Alcohol and Organic-Acid Molecules by Water Surfaces, J. Phys. Chem., 95, 6329-6336, 1991.

Jayne, J.T., Duan, S. X., Davidovits, P., Worsnop, D. R., Zahniser, M. S., and Kolb, C. E.: Uptake of gas-phase aldehydes by water surfaces, J. Phys. Chem., 96, 5452-5460, 1992.

Jones, C. C, Chughtai, A. R., Murugaverl, B., and Smith, D. M.: Effects of air/fuel combustion ratio on the polycyclic aromatic hydrocarbon content of carbonaceous soots from selected fuels, Carbon, 42, 2471-2484, 2004.

Jorgensen, W. L., Chandrasekhar, J., Madura, J. D., Impey, R. W., and Klein, M. L.: Comparison of Simple Potential Functions for Simulating Liquid Water, J. Chem. Phys., 79, 926-935, 1983.

Jungwirth, P. and Tobias, D. J.: Specific ion effects at the air/water interface, Chem. Rev., 106, 1259-1281, 2006.

Kalberer, M., Paulsen, D., Sax, M., Steinbacher, M., Dommen, J., Prevot, A. S. H., Fisseha, R.,Weingartner, E., Frankevich, V.,
Zenobi, R., and Baltensperger, U.: Identification of Polymers as Major Components of Atmospheric Organic Aerosols, Science, Washington DC, USA, 303, 1659-1662, 2004.

Kane, S. M., Caloz, F., and Leu, M.-T.: Heterogeneous Uptake of Gaseous $\mathrm{N}_{2} \mathrm{O}_{5}$ by $\left(\mathrm{NH}_{4}\right)_{2} \mathrm{SO}_{4}, \mathrm{NH}_{4} \mathrm{HSO}_{4}$, and $\mathrm{H}_{2} \mathrm{SO}_{4}$ Aerosols, J. Phys. Chem. A 105, 6465-6470, 2001.

Karagulian, F. and Rossi, M. J.: The heterogeneous kinetics of $\mathrm{NO}_{3}$ on atmospheric mineral dust surrogates, Phys. Chem. Chem. Phys. , 7, 3150-3162, 2005.

Karagulian, F., Santschi, C., and Rossi, M. J.: The heterogeneous chemical kinetics of $\mathrm{N}_{2} \mathrm{O}_{5}$ on $\mathrm{CaCO}_{3}$ and other atmospheric mineral dust surrogates, Atmos. Chem. Phys., 6, 1373-1388, doi:10.5194/acp-6-1373-2006, 2006a.

Karagulian, F. and Rossi, M. J.: The heterogeneous decomposition of ozone on atmospheric mineral dust surrogates at ambient temperatures, Int. J. Chem. Kin., 38, 407-419, 2006 b.

Karagulian, F. and Rossi, M. J.: Heterogeneous chemistry of the $\mathrm{NO}_{3}$ free radical and $\mathrm{N}_{2} \mathrm{O}_{5}$ on decane flame soot at ambient temperature: Reaction products and kinetics, J. Phys. Chem. A, 111, 1914-1926, 2007.

Karpovich, D. S. and Ray, D.: Adsorption of dimethyl sulfoxide to the liquid/vapor interface of water and the thermochemistry of transport across the interface, J. Phys. Chem. B, 102, 649-652, 1998.

Katrib, Y., Le Calve, S., and Mirabel, P.: Uptake measurements of dibasic esters by water droplets and determination of their Henry's law constants, J. Phys. Chem. A, 107, 11433-11439, 2003.

Katrib, Y., Martin, S. T., Hung, H. M., Rudich, Y., Zhang, H. Z., Slowik, J. G., Davidovits, P., Jayne, J. T., and Worsnop, D. R.: Products and mechanisms of ozone reactions with oleic acid for aerosol particles having core-shell morphologies, J. Phys. Chem. A, 108, 6686-6695, 2004.

Katrib, Y., Biskos, G., Buseck, P. R., Davidovits, P., Jayne, J. T., Mochida, M., Wise, M. E., Worsnop, D. R., and Martin, S. T.: Ozonolysis of mixed oleic-acid/stearic-acid particles: Reaction kinetics and chemical morphology, J. Phys. Chem. A, 109, 10910-10919, 2005a.

Katrib, Y., Martin, S. T., Rudich, Y., Davidovits, P., Jayne, J. T., and Worsnop, D. R.: Density changes of aerosol particles as a result of chemical reaction, Atmos. Chem. Phys., 5, 275-291, doi:10.5194/acp-5-275-2005, 2005b.

Kemball, C. and Rideal, E. K.: The Adsorption of Vapours on Mercury .1. Non-Polar Substances, Proc. Roy. Soc. Lond. Math. Phys., 187, 53-73, 1946.

King, D. A. and Wells, M. G.: Molecular beam investigation of adsorption kinetics on bulk metal targets: Nitrogen on tungsten, Surf. Sci., 29, 454-482, 1972.

Kleffmann, J., Becker, K. H., and Lackhoff, M., et al. : Heterogeneous conversion of $\mathrm{NO}_{2}$ on carbonaceous surfaces, Phys. Chem. Chem. Phys., 1, 5443-5450, 1999.

Knopf, D. A., Anthony, L. M., and Bertram, A. K.: Reactive uptake of $\mathrm{O}_{3}$ by multicomponent and multiphase mixtures containing oleic acid, J. Phys. Chem. A, 109, 5579-5589, 2005.

Knopf, D. A., Mak, J., Gross, S., and Bertram, A. K.: Does Atmospheric Processing of Saturated Hydrocarbon Surfaces by NO Lead to Volatilization? Geophys. Res. Lett., 33, L17816, doi:10.1029/2006GL026884, 2006.

Kolb, C. E., Worsnop, D., Zahniser, M. S., Davidovits, P., Keyser, 
L. F., Leu, M.-T., Molina, M. J., Hanson, D. R., Ravishankara, A. R., Williams, L. R., and Tolbert, M. A.: Laboratory Studies of Atmospheric Heterogeneous Chemistry, in: Progress and Problems in Atmospheric Chemistry, edited by: Barker, J. R., Adv. Series Phys. Chem., 3 C.-Y. Ng, series editor, World Scientific, Singapore, 771-875, 1995.

Krieger, U. K., Huthwelker, T., Daniel, C., Weers, U., Peter, T., and Lanford, W. A.: Rutherford Backscattering to Study the NearSurface Region of Volatile Liquids and Solids, Science, 295, 1048-1050, 2002.

Krivacsy, Z., Kiss, G., Varga, B., Galambos, I., Sarvari, Z., Gelencser, A., Molnar, A., Fuzzi, S., Facchini, M. C., Zappoli, S., Andracchio, A., Alsberg, T., Hansson, H. C., and Persson, L.: Study of humic-like substances in fog and interstitial aerosol by size-exclusion chromatography and capillary electrophoresis, Atmos. Environ., 34, 4273-4281, 2000.

Kulmala, M. and Vesala, T.: Condensation in the Continuum Regime, J. Aerosol Sci., 22, 337-346, 1991.

Kulmala, M., Vesala, T., and Wagner, P. E.: An analytical expression for the rate of binary condensational growth, Proc. R. Soc. Lond. A, 441, 589-605, 1993a.

Kulmala, M., Laaksonen, A., Korhonen, P., Vesala, T., Ahonen, T., and Barrett, J. C.: The effect of atmospheric nitric acid vapour on CCN activation, J. Geophys Res., 98, 22949-22958, 1993b.

Kulmala, M. and Wagner, P. E.: Mass accommodation and uptake coefficients - a quantitative comparison, J. Aerosol Sci., 32, 833$841,2001$.

Kuo, I. F. W. and Mundy, C. J.: An ab initio molecular dynamics study of the aqueous liquid-vapor interface, Science, 303, 658660, 2004.

Kwamena, N. O. A., Thornton, J. A., and Abbatt, J. P. D.: Kinetics of surface-bound benzo $[\alpha]$ pyrene and ozone on solid organic and salt aerosols, J. Phys. Chem. A, 108, 11626-11634, 2004.

Kwamena, N. O. A., Earp, M. E., Young, C. J., and Abbatt, J. P. D.: Kinetic and product yield study of the heterogeneous gassurface reaction of anthracene and ozone, J. Phys. Chem. A, 110, 3638-3646, 2006.

Kwan, A. J., Crounse, J. D., Clarke, A. D., Shinozuka, Y., Anderson, B. E., Crawford, J. H., Avery, M. A., McNaughton, C. S., Brune, W. H., Singh, H. B., and Wennberg, P. O.: On the Flux of Oxygenated Volatile Organic Compounds from Organic Aerosol Oxidation, Geophys. Res. Lett. 33, L15815, doi:10.1029/2006GL026144, 2006.

Laaksonen, A., Vesala. T., Kulmala, M., Winkler, P. M., and Wagner, P. E.: Commentary on cloud modelling and the mass accommodation coefficient of water, Atmos. Chem. Phys., 5, 461-464, doi:10.5194/acp-5-461-2005, 2005.

Lambe, A. T., Miracolo, M. A., Hennigan, C. J., Robinson, A. L., and Donahue, N. M.: Effective Rate Constants and Uptake Coefficients for the Reactions of Organic Molecular Markers ( $n-$ Alkanes, Hopanes, and Steranes) in Motor Oil and Diesel Primary Organic Aerosols with Hydroxyl, Environ. Sci. Technol., 43, 8794-8800, 2009.

Lammel, G. and Novakov, T.: Water Nucleation Properties Of Carbon-Black And Diesel Soot particles, Atmos. Environ., 29, 813-823, 1995.

Laskin, A., Wang, H., Robertson, W. H., Cowin, J. P., Ezell, M. J., and Finlayson-Pitts, B. J.: A New Approach to Determining GasParticle Reaction Probabilities and Application to the Heteroge- neous Reaction of Deliquesced Sodium Chloride Particles with Gas-Phase Hydroxyl Radicals, J. Phys. Chem. A, 110 1061910627, 2006.

Lawrence, J. R., Glass, S. V., and Nathanson, G. M.: Evaporation of Water through Butanol Films at the Surface of Supercooled Sulfuric Acid, J. Phys. Chem. A, 109, 7449-7457, 2005 a.

Lawrence, J. R., Glass, S. V., Park, S.-C., and Nathanson, G. M.: Surfactant Control of Gas Uptake: Effect of Butanol Films on $\mathrm{HCl}$ and $\mathrm{HBr}$ Entry into Supercooled Sulfuric Acid, J. Phys. Chem. A, 109, 7458-7465, 2005 b.

Lee, C. Y. and Scott, H. L.: The Surface-Tension Of Water - A Monte-Carlo Calculation Using An Umbrella Sampling Algorithm, J. Chem. Phys., 73, 4591-4596, 1980.

Lemberg, H. L. and Stillinger, F. H.: Central-Force Model For Liquid Water, J. Chem. Phys., 62, 1677-1690, 1975.

Li, L., Chen, Z. M., Zhang, Y. H., Zhu, T., Li, J. L. and Ding, J.: Kinetics and mechanism of heterogeneous oxidation of sulfur dioxide by ozone on surface of calcium carbonate, Atmos. Chem. Phys., 6, 2453-2464, doi:10.5194/acp-6-2453-2006, 2006.

Li, Y., Davidovits, P., Shi, Q., Jayne, J., Kolb, C., and Worsnop, D.: Mass and Thermal Accommodation Coefficients of $\mathrm{H}_{2} \mathrm{O}(\mathrm{g})$ on Liquid Water as a Function of Temperature, J. Phys. Chem. A, 105, 10627-10634, 2001.

Lie, G. C., Clementi, E., and Yoshimine, M.: Study of the structure of molecular complexes. XIII. Monte Carlo simulation of liquid water with a configuration interaction pair potential, J. Chem. Phys., 64, 2314-2323, 1976.

Lie, G. C., Grigoras, S., Dang, L. X., Yang, D. Y., and McLean, A. D.: Monte-Carlo Simulation of the Liquid-Vapor Interface of Water Using an Ab-Initio Potential, J. Chem. Phys., 99, 39333937, 1993.

Liu, Y., Gibson, E. R., Cain, J. P., Wang, H., Grassian, V. H., and Laskin, A.: Kinetics of Heterogeneous Reaction of $\mathrm{CaCO}_{3}$ Particles with Gaseous $\mathrm{HNO}_{3}$ over a Wide Range of Humidity, J. Phys. Chem. A, 112, 1561-1571, 2008.

Livingston, F. E. and George, S. M.: Effect of $\mathrm{HNO}_{3}$ and $\mathrm{HCl}$ on $\mathrm{D}_{2} \mathrm{O}$ Desorption Kinetics from Crystalline $\mathrm{D}_{2} \mathrm{O}$ Ice, J. Phys. Chem. A, 102, 10280-10288, 1998.

Longfellow, C. A., Ravishankara, A. R., Hanson, D. R., et al.: Reactive and nonreactive uptake on hydrocarbon soot: $\mathrm{HNO}_{3}, \mathrm{O}_{3}$, and $\mathrm{N}_{2} \mathrm{O}_{5}$, J. Geophys. Res.-Atmos., 105, 24345-24350, 2000.

Lu, H., McCartney, S. A., Chonde, M., Smyla, D., and Sadtchenko, V.: Fast thermal desorption spectroscopy study of morphology and vaporization kinetics of polycrystalline ice films, J. Chem. Phys., 125, 044709, doi:10.1063/1.2212395, 2006.

Magee, N., Moyle, A. M., and Lamb, D.: Experimental determination of the deposition coefficient of small cirrus-like ice crystals near $-50^{\circ} \mathrm{C}$, Geopyhs. Res. Lett., 33, L17813, doi:10.1029/2006GL026665, 2006.

Magi, L., Schweitzer, F., Pallares, C., Cherif, S., Mirabel, P., and George, C.: Investigation of the uptake rate of ozone and methyl hydroperoxide by water surfaces, J. Phys. Chem. A., 101, 49434949, 1997.

Mahiuddin, S., Minofar, B., Borah, J. M., Das, M. R., and Jungwirth, P.: Propensities of oxalic, citric, succinic, and maleic acids for the aqueous solution/vapour interface: Surface tension measurements and molecular dynamics simulations, Chem. Phys. Lett., 462, 217-221, 2008.

Mashburn, C. D., Frinak, E. K., and Tolbert, M. A.: Heteroge- 
neous uptake of nitric acid on $\mathrm{Na}$-montmorillonite clay as a function of relative humidity, J. Geophys. Res., 111, D15213, doi:10.1029/2005JD006525, 2006.

Matsumoto, M. and Kataoka, Y.: Study on Liquid Vapor Interface of Water .1. Simulational Results of Thermodynamic Properties and Orientational Structure, J. Chem. Phys., 88, 3233-3245, 1988.

Matsumoto, M., Takaoka, Y., and Kataoka, Y.: Liquid Vapor Interface of Water-Methanol Mixture .1. Computer-Simulation, J. Chem. Phys., 98, 1464-1472, 1993.

Matsumoto, M.: Molecular dynamics simulation of interphase transport at liquid surfaces, Fluid Phase Equilibria, 125, 195203, 1996.

Matsuoka, O., Clementi, E., and Yoshimine, M.: CI study of the water dimer potential surface, J. Chem. Phys., 64, 1351-1362, 1976.

McFiggans, Artaxo, G. P., Baltensperger, U., Coe, H., Facchini, M. C., Feingold, G., Fuzzi, S., Gysel, M., Laaksonen, A., Lohmann, U., Mentel, T. F., Murphy, D. M., O’Dowd, C. D., Snider, J. R., and Weingartner, E.: The Effect of Physical and Chemical Aerosol Properties on Warm Cloud Droplet Activation, Atmos. Chem. Phys., 6, 2593-2649, doi:10.5194/acp-6-2593, -2006m 2006.

McMurry, P. H., Takano, H., and Anderson, G. R.: Study of the Ammonia (Gas)-Sulfuric Acid (Aerosol) Reaction Rate, Environ. Sci. Technol., 17, 347-352, 1983.

McNeill, V. F., Loerting, T., Geiger, F. M., Trout, B. L., and Molina, M. J.: Hydrogen chloride-induced surface disordering on ice, Proc. Natl. Acad. Sci. USA, 103, 9422-9427, 2006a.

McNeill, V. F., Patterson, J., Wolfe, G. M., and Thornton, J. A.: The effect of varying levels of surfactant on the reactive uptake of $\mathrm{N}_{2} \mathrm{O}_{5}$ to aqueous aerosol, Atm. Chem. Phys., 6, 1635-1644, $2006 \mathrm{~b}$.

McNeill, V. F, Geiger, F. M., Loerting, Th., Trout, B. L., Molina, L. T., and Molina, M. J.: Interaction of hydrogen chloride with ice surfaces: The effects of grain size, surface roughness and surface disorder, J. Phys. Chem. A, 111, 6274-6284, 2007.

McNeill, V. F., Yatavelli, R. L. N., Thornton, J. A., Stipe, C. B., and Landgrebe, O.: Heterogeneous $\mathrm{OH}$ oxidation of palmitic acid in single component and internally mixed aerosol particles: vaporization and the role of particle phase, Atmos. Chem. Phys., 8, 5465-5476, doi:10.5194/acp-8-5465-2008, 2008.

Mentel, T. F., Sohn, M., and Wahner, A.: Nitrate effect in the heterogeneous hydrolysis of dinitrogen pentoxide on aqueous aerosols, Phys. Chem. Chem. Phys., 1, 5451-5457, DOI: 10.1039/a905338g, 1999.

Miet, K., Le Menach, K., Flaud, P.-M., Budzinski, H. and Villenave, E.: Heterogeneous reactivity of pyrene and 1-nitropyrene with $\mathrm{NO}_{2}$ : Kinetics, product yields and mechanism, Atmos. Environ., 43, 837-843, 2009a.

Miet, K., Le Menach, K., Flaud, P.-M., Budzinski, H. and Villenave, E.: Heterogeneous reactions of ozone with pyrene, 1hydroxypyrene and 1-nitropyrene adsorbed on particles, Atmos. Environ., 43, 3699-3707, 2009b.

Miller, C. A., Abbott, N. L., and de Pablo, J. J.: Surface Activity of Amphiphilic Helical beta-Peptides from Molecular Dynamics Simulation, Langmuir, 25, 2811-2823, 2009.

Miller, R. L., Tegen, I., and Perlwitz, J.: Surface radiative forcing by soil dust aerosols and the hydrologic cycle, J. Geophys. Res. Atmos., 109, D04203, doi:10.1029/2003JD004085, 2004.
Minofar, B., Jungwirth, P., Das, M. R., Kunz, W., and Mahiuddin, S.: Propensity of formate, acetate, benzoate, and phenolate for the aqueous solution/vapor interface: Surface tension measurements and molecular dynamics simulations, J. Phys. Chem. C, 111, 8242-8247, 2007.

Mmereki, B. T., Hicks, J. M., and Donaldson, D. J.: Adsorption of atmospheric gases at the air-water interface. 3: Methylamines, J. Phys. Chem. A, 104, 10789-10793, 2000.

Mmereki, B. T., Chaudhuri, S. R., and Donaldson, D. J.: Enhanced uptake of PAHs by organic-coated aqueous surfaces, J. Phys. Chem. A, 107, 2264-2269, 2003.

Mmereki, B. T., Donaldson, D. J., Gilman, J. B., Eliason, T. L., and Vaida, V.: Kinetics and products of the reaction of gas-phase ozone with anthracene adsorbed at the air-aqueous interface, Atmos. Environ., 38, 6091-6103, 2004.

Mogili, P. K., Kleiber, P. D., Young, M. A., and Grassian, V. H.: Heterogeneous Uptake of Ozone on Reactive Components of Mineral Dust Aerosol: An Environmental Aerosol reaction Chamber Study. J. Phys. Chem. A, 110, 13799-13807, 2006a..

Mogili, P.K., Kleiber, P. D., Young, M. A., and Grassian, V. H.: $\mathrm{N}_{2} \mathrm{O}_{5}$ hydrolysis on the components of mineral dust and sea salt aerosol: comparison study in an environmental aerosol reaction chamber, Atmos. Environ., 40, 7401-7408, 2006 b.

Möhler, O., Benz, S., Saathoff, H., Schnaiter, M.,Wagner, R., Schneider, J., Walter, S., Ebert, V., and Wagner, S.: The effect of organic coating on the heterogeneous ice nucleation efficiency of mineral dust aerosols, Environ. Res. Lett., 3, 025007, doi:10.1088/1748-9326/3/2/025007, 2008.

Moise, T. and Rudich, Y.: Reactive uptake of ozone by proxies for organic aerosols: Surface versus bulk processes, J. Geophys. Res.-Atmos., 105, 14667-14676, 2000.

Moise, T. and Rudich, Y.: Reactive uptake of $\mathrm{Cl}$ and $\mathrm{Br}$ atoms by organic surfaces - a perspective on the processing of organic aerosols by tropospheric oxidants, Geophys. Res. Lett., 28, 4083-4086, 2001.

Moise, T. and Rudich, Y.: Reactive uptake of ozone by aerosolassociated unsaturated fatty acids: Kinetics, mechanism, and products, J. Phys. Chem. A, 106, 6469-6476, 2002.

Moise, T., Rudich, Y., Rousse, D., and George, C.: Multiphase decomposition of novel oxygenated organics in aqueous and organic media, Environ. Sci. Technol., 39, 5203-5208, 2005.

Molina, M. J., Ivanov, A. V., Trakhtenberg, S., and Molina, L. T.: Atmospheric evolution of organic aerosol, Geophys. Res. Lett., 31, L22104, doi:10.1029/2004GL020910, 2004.

Monge, M. E., D’Anna, B., Mazri, L., Giroir-Fendler, A., Ammann, M., Donaldson, D. J., and George, C.: Light changes the atmospheric reactivity of soot, Proc. Nat. Acad. Sci., 107, 6605609, 2010.

Morita, A.: Molecular dynamics study of mass accommodation of methanol at liquid-vapor interfaces of methanol/water binary solutions of various concentrations, Chem. Phys. Lett., 375, 1-8, 2003a.

Morita, A. and Garrett, B. C.: Molecular theory of mass transfer kinetics and dynamics at gas-water interface, Fluid Dynam. Res., 40, 459-473, 2008.

Morita, A., Sugiyama, M., Kameda, H., Koda, S., and Hanson, D. R.: Mass accommodation coefficient of water: Molecular dynamics simulation and revised analysis of droplet train/flow reactor experiment, J. Phys. Chem. B, 108, 9111-9120, 2004a. 
Morita, A., Kanaya, Y., and Francisco, J. S.: Uptake of the $\mathrm{HO}_{2}$ radical by water: Molecular dynamics calculations and their implications for atmospheric modeling, J. Geophys. Res.-Atmos., 109, D09201, doi:10.1029/2003JD004240, 2004b.

Morris, J. W., Davidovits, P., Jayne, J. T., Shi, Q., Kolb, C. E., Worsnop, D. R., Barney, W. S., Jimenez, J., and Cass, G.: Kinetics of submicron oleic acid aerosols with ozone: A novel aerosol mass spectrometric technique, Geophys. Res. Lett., 29, 1357, doi:1029/2002GL014692, 2002.

Mozurkewich, M.: Effect of competitive adsorption on polar stratospheric cloud reactions, Geophys. Res. Lett., 20, 355-358, 1993.

Mozurkewich, M., McMurry, P. H., Gupta, A., et al.: Mass accommodation coefficient for $\mathrm{HO}_{2}$ radicals on aqueous particles, J. Geophys. Res., 92, 4163-4170, 1987.

Muller, B. and Heal, M. R.: The mass accommodation coefficient of ozone on an aqueous surface, Phys. Chem. Chem. Phys., 4, 3365-3369, 2002.

Mundy, C. J., Rousseau, R., Curioni, A., Kathmann, S. M., and Schemer, G. K.: A molecular approach to understanding complex systems: computational statistical mechanics using state-ofthe-art algorithms on terascale computational platforms, edited by: Stevens, R. L., SciDAC 2008: Scientific Discovery through Advanced Computing, 012014, 12014-12014, 2008.

Nagayama, G. and Tsuruta, T.: A general expression for the condensation coefficient based on transition state theory and molecular dynamics simulation, J. Chem. Phys., 118, 1392-1399, 2003.

NASA.: Chemical Kinetics and Photochemical Data for Use in Atmospheric Studies, JPL Publication No. 06-2; Jet Propulsion Laboratory, National Aeronautics and Space Administration, Pasadena, CA, USA, available online at: http://jpldataeval. jpl.nasa.gov/, 2006.

Ndour, M., D’Anna, B., George, C., Ka, O., Balkanski, Y., Kleffmann, J., Stemmler, K., and Ammann, M.: Photoenhanced update of $\mathrm{NO}_{2}$ on mineral dust: laboratory experiments and model simulations, Geophys. Res. Lett. 35, L05812, doi:10.1029/2007GL032006, 2008.

Niesar, U., Corongiu, G., Huang, M. J., Dupuis, M., and Clementi, E.: Preliminary-Observations On A New Water Water Potential, International J. Quant. Chem., 36(S23), 421-443, 1989.

Niesar, U., Corongiu, G., Clementi, E., Kneller, G. R., and Bhattacharya, D. K.: Molecular-Dynamics Simulations of Liquid Water Using the Ncc Ab Initio Potential, J. Phys. Chem., 94, 79497956, 1990.

Nieto-Gligorovski, L., Net, S., Gligorovski, S., Zetzsch, C., Jammoul, A., D'Anna, B., and George, C.: Interactions of ozone with organic surface films in the presence of simulated sunlight: impact on wettability of aerosols, Phys. Chem. Chem. Phys., 10, 2964-2971, doi:10.1039/b717993f, 2008.

Nozière, B. and Esteve, W.: Organic reactions increasing the absorption of sulphuric acid aerosols, Geophys. Res. Lett., 32, L03812, doi:10.1029/2004GL02194, 2005.

Nozière, B. and Esteve, W.: Light-absorbing aldol condensation products in acidic aerosols: Spectra, kinetics, and contribution to the absorption index, Atmos. Environ., 41, 1150-1163, 2007.

Nozière, B., Dziedzic, P., and Córdova, A.: Formation of secondary light-absorbing "fulvic-like" oligomers: A common process in aqueous and ionic atmospheric particles? Geophys. Res. Lett., 34, L21812, doi:10.1029/2007GL031300, 2007.

Oppliger, R., Allanic, A., and Rossi, M. J.: Real-time kinetics of the uptake of $\mathrm{ClONO}_{2}$ on ice and in the presence of $\mathrm{HCl}$ in the temperature range $160 \mathrm{~K}=T=200 \mathrm{~K}$, J. Phys. Chem. A, 101, 1903-1911, 2007.

Oum, K. W., Lakin, M. J., DeHaan, D. O., Brauers, T., and Finlayson-Pitts, B. J.: Formation of Molecular Chlorine from the Photolysis of Ozone and Aqueous Sea-Salt Particles, Science, 279, 74-76, 1998a.

Oum, K. W., Lakin, M. J., and Finlayson-Pitts, B. J.: Bromine Activation in the Troposphere by the Dark Reaction of $\mathrm{O}_{3}$ and Seawater Ice, Geophys. Res. Lett., 25, 3923, doi:10.1029/1998GL900078,1998b.

Padró, L. T., Asa-Awuku, A., Morrison, R., and Nenes, A.: Inferring thermodynamic properties from $\mathrm{CCN}$ activation experiments: single-component and binary aerosols, Atmos. Chem. Phys., 7, 5263-5274, doi:10.5194/acp-7-5263-2007, 2007.

Parent, P. and Laffon, C.: Adsorption of $\mathrm{HCl}$ on the water ice surface studied by X-ray absorption spectroscopy, J. Phys. Chem. B, 109, 1547-1553, 2005.

Park, J., Gomez, A. L., Walser, M. L., Lin, A., and Nizkorodov, S. A.: Ozonolysis and photolysis of alkene-terminated selfassembled monolayers on quartz nanoparticles: implications for photochemical aging of organic aerosol particles, Phys. Chem. Chem. Phys., 8, 2506-2512, 2006.

Park, S.-C., Burden, D. K., and Nathanson, G. M.: The Inhibition of $\mathrm{N}_{2} \mathrm{O}_{5}$ Hydrolysis in Sulfuric Acid by 1-Butanol and 1-Hexanol Surfactant Coatings, J. Phys. Chem. A., 111, 2921-2929, 2007.

Partay, L. B., Jedlovszky, P., Hoang, P. N. M., Picaud, S., and Mezei, M.: Free-energy profile of small solute molecules at the free surfaces of water and ice, as determined by cavity insertion Widom calculations, J. Phys. Chem. C, 111, 9407-9416, 2007.

Patel, S., Zhong, Y., Bauer, B. A., and Davis, J. E.: Interfacial Structure, Thermodynamics, and Electrostatics of Aqueous Methanol Solutions via Molecular Dynamics Simulations Using Charge Equilibration Models, J. Phys. Chem. B, 113, 9241-9254, 2009.

Paul, S. and Chandra, A.: Dynamics of water molecules at liquidvapour interfaces of aqueous ionic solutions: effects of ion concentration, Chem. Phys. Lett., 373, 87-93, 2003.

Paul, S. and Chandra, A.: Binding of hydrogen bonding solutes at liquid-vapour interfaces of molecular fluids, Chem. Phys. Lett., 400, 515-519, 2004.

Pegram, L. M. and Record, M. T.: Partitioning of atmospherically relevant ions between bulk water and the water/vapor interface, Proc. Natl. Acad. Sci., 103, 14278-14281, 2006.

Perraudin, E., Budzinski, H., and Villenave, E.: Kinetic study of the reactions of ozone with polycyclic aromatic hydrocarbons adsorbed on atmospheric model particles, J. Atmos. Chem., 56, 57-82, 2007.

Persiantseva, N. M., Popovicheva, O. B., Shonija, N. K., et al.: Wetting and hydration of insoluble soot particles in the upper troposphere, J. Environ. Monitor., 6, 939-945, 2004.

Peter, T., Marcolli, C., Spichtinger, P., Corti, T., Baker, M. B., and Koop, T.: When dry air is too humid, Science, 314, 1399-1402, 2006.

Petersen, P. B., Johnson, J. C., Knutsen, K. P., and Saykally, R. J.: Direct experimental validation of the Jones-Ray effect, Chem. Phys. Lett., 397, 46-50, 2004.

Petersen, P. B. and Saykally, R. J.: On the Nature of Ions at the Liquid Water Surface, Ann. Rev. Phys. Chem., 57, 333-364, 2006.

Petzold, A., Gysel, M., Vancassel, X., Hitzenberger, R., Puxbaum, 
H., Vrochticky, S., Weingartner, E., Baltensperger, U., and Mirabel, P.: On the effects of organic matter and sulphurcontaining compounds on the $\mathrm{CCN}$ activation of combustion particles, Atmos. Chem. Phys., 5, 3187-3203, doi:10.5194/acp-53187-2005, 2005.

Pfrang, C., Shiraiwa, M., and Pöschl, U.: Coupling aerosol surface and bulk chemistry with a kinetic double layer model (K2-SUB): oxidation of oleic acid by ozone, Atmos. Chem. Phys., 10, 45374557, doi:10.5194/acp-10-4537-2010, 2010.

Pohorille, A. and Benjamin, I.: Molecular-Dynamics of Phenol at the Liquid Vapor Interface of Water, J. Chem. Phys., 94, 5599$5605,1991$.

Pohorille, A. and Benjamin, I.: Structure and Energetics of Model Amphiphilic Molecules at the Water Liquid Vapor Interface a Molecular-Dynamics Study, J. Phys. Chem., 97, 2664-2670, 1993a.

Pohorille, A. and Wilson, M. A.: Molecular Structure of Aqueous Interfaces, Journal of Molecular Structure: Theochem, 284, 271298, 1993b.

Pöschl, U.: Formation and decomposition of hazardous chemical components contained in atmospheric aerosol particles, Journal of Aerosol Medicine-Deposition Clearance and Effects In The Lung, 15, 203-212, 2002.

Pöschl, U., Letzel, T., Schauer, C., and Niessner, R.: Interaction of ozone and water vapor with spark discharge soot aerosol particles coated with benzo[a]pyrene: $\mathrm{O}_{3}$ and $\mathrm{H}_{2} \mathrm{O}$ adsorption, benzo[a]pyrene degradation, and atmospheric implications, J. Phys. Chem. A, 105, 4029-4041, 2001.

Pöschl, U., Rudich, Y., and Ammann, M.: Kinetic model framework for aerosol and cloud surface chemistry and gas-particle interactions - Part 1: General equations, parameters, and terminology, Atmos. Chem. Phys., 7, 5989-6023, doi:10.5194/acp-75989-2007, 2007.

Pradhan, M., Kyriakou, G., Archibald, A. T., Papageorgiou, A. C., Kalberer, M., and Lambert, R. M.: Heterogeneous uptake of gaseous hydrogen peroxide by Gobi and Saharan dust aerosols: a potential missing sink for $\mathrm{H}_{2} \mathrm{O}_{2}$ in the troposphere, Atmos. Chem. Phys., 10, 7127-7136, doi:10.5194/acp-10-71272010, 2010.

Pratt, L. R. and Pohorill e, A.: Hydrophobic effects and modeling of biophysical aqueous solution interfaces, Chem. Rev., 102, 26712691, 2002.

Pratte, P., van den Bergh, H., and Rossi, M. J.: The kinetics of $\mathrm{H}_{2} \mathrm{O}$ vapor condensation and evaporation on different types of ice in the range 130-210K, J. Phys. Chem. A, 110, 3042-3058, 2006.

Preszler Prince, A., Grassian, V. H., Kleiber, P., and Young, M. A.: Heterogeneous conversion of calcite aerosol by nitric acid, Phys. Chem. Chem. Phys., 9, 622-634, 2007.

Quinlan, M. A., Reihs, C. M., Golden, D. M., and Tolbert, M. A.: Heterogeneous Reactions on model polar stratospheric cloud surfaces, J. Phys. Chem., 94, 3255-3260, 1990.

Rahman, A., Stillinger, F. H., and Lemberg, H. L.: Study of a Central Force Model for Liquid Water by Molecular-Dynamics, J. Chem. Phys., 63, 5223-5230, 1975.

Remorov, R. G. and Bradwell, M. W.: Langmuir Approach in the Study of Interfacial Mass Transfer, Surf. Sci., 585, 59-65, 2005.

Remorov, R. G. and George, C.: Analysis of chemical kinetics at the gas-aqueous interface for submicron aerosols, Phys. Chem. Chem. Phys., 8, 4897-4901, 2006.
Riipinen, I., Koponen, I. K., Frank, G. P., Hyvärinen, A.-P., Vanhanen, J., Lihavainen, H., Lehtinen, K. E. J., Bilde, M. and Kulmala, M.: Adipic and malonic acid aqueous solutions: surface tensions and saturation vapor pressures, J. Phys. Chem. A., 111, 12995-13002, 2007.

Robbins, R. C. and Cadle, R. D.: Kinetics of the Reaction Between Gaseous Ammonia and Sufuric Acid Droplets in an Aerosol, J. Phys. Chem., 62, 469-471, 1958.

Roberts, J. M., Osthoff, H. D., Brown, S. S., and Ravishankara, A. R.: $\mathrm{N}_{2} \mathrm{O}_{5}$ Oxidizes Chloride to $\mathrm{Cl}_{2}$ in Acidic Atmospheric Aerosol, Science, 321, 1059-1059, 2008.

Robinson, G. N., Worsnop, D. R., Jayne, J. T., Kolb, C. E., and Davidovits, P.: Heterogeneous uptake of $\mathrm{ClONO}_{2}$ and $\mathrm{N}_{2} \mathrm{O}_{5}$ by sulfuric acid solutions, J. Geophys. Res., 102, 3583-3602, 1997.

Roeselova, M., Jungwirth, P., Tobias, D. J., and Gerber, R. B.: Impact, trapping, and accommodation of hydroxyl radical and ozone at aqueous salt aerosol surfaces. A molecular dynamics study, J. Phys. Chem. B, 107, 12690-12699, 2003.

Roeselova, M., Vieceli, J., Dang, L. X., Garrett, B. C., and Tobias, D. J.: Hydroxyl radical at the air-water interface, J. Am. Chem. Soc., 126, 16308-16309, 2004.

Roth, C. M., Goss, K.-U., and Schwarzenbach, R. P.: Adsorption of a diverse set of organic vapors on the bulk water surface, J. Coll. Int. Sci., 252, 21-30, 2002.

Rudich, Y.: Laboratory perspectives on the chemical transformations of organic matter in atmospheric particles, Chem. Rev., 103, 5097-5124, 2003.

Rudich, Y., Donahue, N. M., and Menache, M. G.: Aging of Organic Aerosol: Bridging the Gap Between Laboratory and Field Studies, Ann. Rev. Phys. Chem., 58: 321-352, 2007.

Rudich, Y., Talukdar, R., and Ravishankara, A. R.: Reactive uptake of $\mathrm{NO}_{3}$ on pure water and ionic solutions, J. Geophys. Res., 101, 21023-21032, 1996.

Rudolf, R., Vrtala, A., Kulmala, M., Vesala, T., and Wagner, P. E.: Experimental study of sticking probabilities for condensation of nitric acid-water vapor mixtures, J. Aerosol Sci., 32, 913-932, 2001.

Reutter, P. H. Su, Trentmann, J., Simmel, M., Rose, D., Gunthe, S. S., H. Wernli, H., Andreae, M. O., and Pöschl, U.: Aerosoland updraft-limited regimes of cloud droplet formation: in?uence of particle number, size and hygroscopicity on the activation of cloud condensation nuclei (CCN), Atmos. Chem. Phys., 9, 70677080, doi:10.5194/acp-9-7067-2009, 2009.

Russell, L. M. Hawkins, L. N. Frossard, A. A., Quinn, P. K., and Bates, T. S.: Carbohydrate-like composition of submicron atmospheric particles and their production from ocean bubble bursting, Proc. Natl. Acad. Sci., 107, 6652-6657, 2010.

Saathoff, H., Naumann, K.-H., Riemer, N., et al.: The loss of $\mathrm{NO}_{2}, \mathrm{HNO}_{3}, \mathrm{NO}_{3} / \mathrm{N}_{2} \mathrm{O}_{5}$, and $\mathrm{HO}_{2} / \mathrm{HOONO}_{2}$ on soot aerosol: A chamber and modeling study, Geophys. Res. Lett., 28, 19571960, 2001.

Sage, A. M., Weitkamp, E. A., Robinson, A. L., and Donahue, N. M.: Reactivity of oleic acid in organic particles: changes in oxidant uptake and reaction stoichiometry with particle oxidation, Phys. Chem. Chem. Phys., 11, 7951-7962, 2009.

Salgado, M. S. and Rossi, M. J.: Flame soot generated under controlled combustion conditions: Heterogeneous reaction of $\mathrm{NO}_{2}$ on hexane soot, Int. J. Chem. Kin., 34, 620-631, 2002.

Sander, R.: Compilation of Henry's Law Constants for Inorganic 
and Organic Species of Potential Importance in Environmental

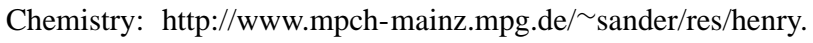
html, 1999.

Santschi, C. and Rossi, M. J.: The uptake of $\mathrm{CO}_{2}, \mathrm{SO}_{2}, \mathrm{HNO}_{3}$ and $\mathrm{HCl}$ on $\mathrm{CaCO}_{3}$ at $300 \mathrm{~K}$ : mechanism and the role of adsorbed water, J. Phys. Chem. A 110, 6789-6802, 2006.

Saul, T. D., Tolocka, M. P., and Johnston, M. V.: Reactive uptake of nitric acid onto sodium chloride aerosols across a wide range of humidities, J. Phys. Chem. A, 110, 7614-7620, 2006.

Schenter, G. K., Garrett, B. C., and Truhlar, D. G.: Generalized transition state theory in terms of the potential of mean force, J. Chem. Phys., 119, 5828-5833, 2003.

Schutze, M. and Herrmann, H.: Determination of phase transfer parameters for the uptake of $\mathrm{HNO}_{3}, \mathrm{~N}_{2} \mathrm{O}_{5}$ and $\mathrm{O}_{3}$ on single aqueous drops, Phys. Chem. Chem. Phys., 4, 60-67, 2002.

Schweitzer, F., Mirabel, P., and George, C.: Multiphase Chemistry of $\mathrm{N}_{2} \mathrm{O}_{5}, \mathrm{ClNO}_{2}$ and $\mathrm{BrNO}_{2}$, J. Phys. Chem. A, 102, 39423952, 1998.

Seinfeld, J. H. and Pandis, S. N.: Atmospheric Chemistry and Physics: from air pollution to climate change, Wiley, New York, USA, 610-611, 1998.

Seisel, S., Börensen, C., Vogt, R., and Zellner, R.: The heterogeneous reaction of $\mathrm{HNO}_{3}$ on mineral dust and $\gamma$ alumina surfaces : a combined Knudsen cell and DRIFTS study, Phys. Chem. Chem. Phys., 6, 5498-5508, 2004.

Shantz, N. C., Chang, R. Y.-W., Slowik, J. G., Vlasenko, A., Abbatt, J. P. D., and Leaitch, W. R.: Slower CCN growth kinetics of anthropogenic aerosol compared to biogenic aerosol observed at a rural site, Atmos. Chem. Phys., 10, 299-312, doi:10.5194/acp10-299-2010, 2010.

Shaw, R. A. and Lamb, D.: Experimental determination of the thermal accommodation and condensation co-efficients of water, J. Chem. Phys., 111, 10659-10663, 1999.

Shi, Q., Li, Y. Q., Davidovits, P., Jayne, J. T., Worsnop, D. R., Mozurkewich, M., and Kolb, C. E.: Isotope exchange for gasphase acetic acid and ethanol at aqueous interfaces: A study of surface reactions, J. Phys. Chem. B, 103, 2417-2430, 1999.

Shilling, J. E., Tolbert, M. A., Toon, O. B., Jensen, E. J., Murray, B. J., and Bertram, A. K.: Measurements of the vapor pressure of cubic ice and their implications for atmospheric ice clouds, Geophys. Res. Lett., 33, L17801, doi:10.1029/2006GL026671, 2006.

Shin, J. Y. and Abbott, N. L.: Combining molecular dynamics simulations and transition state theory to evaluate the sorption rate constants for decanol at the surface of water, Langmuir, 17, 8434-8443, 2001.

Shiraiwa, M., Garland, R. M. and Pöschl, U.: Kinetic double-layer model of aerosol surface chemistry and gas-particle interactions (K2-SURF): Degradation of polycyclic aromatic hydrocarbons exposed to $\mathrm{O}_{3}, \mathrm{NO}_{2}, \mathrm{H}_{2} \mathrm{O}, \mathrm{OH}$ and $\mathrm{NO}_{3}$, Atmos. Chem. Phys., 9, 9571-9586, doi:10.5194/acp-9-9571-2009, 2009.

Shiraiwa, M., Pfrang, C., and Pöschl, U.: Kinetic multi-layer model of aerosol surface and bulk chemistry (KM-SUB): the influence of interfacial transport and bulk diffusion on the oxidation of oleic acid by ozone, Atmos. Chem. Phys., 10, 3673-3691, doi:10.5194/acp-10-3673-2010, 2010.

Shonija, N. K., Popovicheva, O. B., Persiantseva, N. M., et al.: Hydration of aircraft engine soot particles under plume conditions: Effect of sulfuric and nitric acid processing, J. Geophys. Res.-
Atmos., 112, D02208, doi:10.1029/2006JD007217, 2007.

Simpson, A. J., Lam, B., Diamond, M. L., Donaldson, D. J., Lefebvre, B. A., Moser, A. Q., Williams, A. J., Larin, N. I., and Kvasha, M. P.: Assessing the organic composition of urban surface films using nuclear magnetic resonance spectroscopy, Chemosphere, 63, 142-152, 2006.

Sjostedt, S., and Abbatt, J. P. D.: Release of gas-phase halogens from sodium halide substrates: heterogeneous oxidation of frozen solutions and desiccated salts by hydroxyl radicals, Environ. Res. Lett., 3, 045007, doi:10.1088/1748-9326/3/4/045007, 2008.

Smith, J. D., Cappa, C. D., Drisdell, W. S., Cohen, R. C., and Saykally, R. J.: Raman Thermometry Measurements of Free Evaporation from Liquid Water Droplets, J. Amer. Chem. Soc., 128, 12892-12898, 2006.

Sokhan, V. P. and Tildesley, D. J.: Molecular dynamics simulation of the non-linear optical susceptibility at the phenol/water/air interface, Faraday Discussions, 104, 193-208, 1996.

Sprik, M. and Klein, M. L.: A Polarizable Model For Water Using Distributed Charge Sites, J. Chem. Phys., 89, 7556-7560, 1988.

Stadler, D., and Rossi, M. J.: The reactivity of $\mathrm{NO}_{2}$ and $\mathrm{HONO}$ on flame soot at ambient temperature: The influence of combustion conditions, Phys. Chem. Chem. Phys., 2, 5420-5429, 2000.

Stemmler, K., Ammann, M., Donders, C., Kleffmann, J., and George, C.: Photosensitized reduction of nitrogen dioxide on humic acid as a source of nitrous acid, Nature, 440, 195-198, 2006.

Stemmler, K., Ndour, M., Elshorbany, Y., Kleffmann, J., D’Anna, B., George, C., Bohn, B., and Ammann, M.: Light induced conversion of nitrogen dioxide into nitrous acid on submicron humic acid aerosol, Atmos. Chem. Phys., 7, 4237-4248, doi:10.5194/acp-7-4237-2007, 2007.

Stemmler, K., Vlasenko, A., Guimbaud, C. and Ammann, M.: The effect of fatty acid surfactants on the uptake of nitric acid to deliquesced $\mathrm{NaCl}$ aerosol, Atmos. Chem. Phys., 8, 5127-5141, doi:10.5194/acp-8-5127-2008, 2008.

Stewart, D. J., Griffiths, P. T., and Cox, R. A.: Reactive uptake coefficients for heterogeneous reaction of $\mathrm{N}_{2} \mathrm{O}_{5}$ with submicron aerosols of $\mathrm{NaCl}$ and natural sea salt, Atmos. Chem. Phys., 4, 1381-1388, doi:10.5194/acp-4-1381-2004, 2004.

Stewart, E., Shields, R. L., and Taylor, R. S.: Molecular dynamics simulations of the liquid/vapor interface of aqueous ethanol solutions as a function of concentration, J. Phys. Chem. B, 107, 2333-2343, 2003.

Stillinger, F. H. and Rahman, A.: ST2 potential, J. Chem. Phys., 60, 1545-1557, 1974.

Stillinger, F. H. and Rahman, A.: Revised central force potentials for water, J. Chem. Phys., 68, 666-670, 1978.

Stipp, S. L. S., Eggleston, C. M., and Nielsen, B. S.: Calcite surface structure observed at microphotographic and molecular scales with atomic force microscopy (AFM), Geochim. Cosmochim. Acta, 58, 3023-3033, 1994.

Styler, S.A., Brigante, M., D’Anna, B., George, C. H., and Donaldson, D. J.: Photoenhanced ozone loss on solid pyrene films, Phys. Chem. Chem. Phys., 11, 7876-7884, 2009.

Sullivan, R. C., Moore, M. J. K., Petters, M. D., Kreidenweis, S. M., Roberts, G. C., and Prather, K. A.: Timescale for hygroscopic conversion of calcite mineral particles through heterogeneous reaction with nitric acid, Phys. Chem. Chem. Phys., 11, 7826-7837, 2009. 
Sumner, A. L., Menke, E. J., Dubowski, Y., Newberg, J. T., Penner, R. M., Hemminger, J. C., Wingen, L. M., Brauers, T., and Finlayson-Pitts, B. J.: The nature of water on surfaces of laboratory systems and implications for heterogeneous chemistry in the troposphere, Phys. Chem. Chem. Phys., 6, 604-613, 2004.

Sun, X. Q., Chang, T. M., Cao, Y., Niwayama, S., Hase, W. L., and Dang, L. X.: Solvation of Dimethyl Succinate in a Sodium Hydroxide Aqueous Solution. A Computational Study, J. Phys. Chem. B, 113, 6473-6477, 2009.

Symington, A.: The Heterogeneous Interaction of Organic Acids with Ice Surfaces at Temperatures of the Upper Troposphere, CPGS Dissertation, Newham College, University of Cambridge, 2006.

Tabazadeh, A. and Turco, R.: A model for heterogeneous chemical processes on the surfaces of ice and nitric acid trihydrate particles, J. Geophys. Res., 98, 12727-12740, 1993.

Takami, A., Kato, S., Shimono, A., and Koda, S.: Uptake coefficient of $\mathrm{OH}$ radical on aqueous surface, Chem. Phys. 231, 215-227, 1998.

Taketani, F., Kanaya, Y., and Akimoto, H.: Kinetics of heterogeneous reactions of $\mathrm{HO}_{2}$ radical at ambient concentration levels with $\left(\mathrm{NH}_{4}\right)_{2} \mathrm{SO}_{4}$ and $\mathrm{NaCl}$ aerosol particles, J. Phys. Chem. A, 112, 2370-2377, 2008.

Taketani, F., Kanaya, Y., Akimoto, H..: Heterogeneous loss of $\mathrm{HO}_{2}$ by $\mathrm{KCl}$, synthetic sea salt, and natural seawater aerosol particles, Atmos. Environ., 43, 1660-1665, 2009.

Tarek, M., Tobias, D. J., and Klein, M. L.: Molecular dynamics investigation of an ethanol-water solution, Physica A, 231, 117122, 1996a.

Tarek, M., Tobias, D. J., and Klein, M. L.: Molecular dynamics investigation of the surface/bulk equilibrium in an ethanol-water solution, Journal of the Chemical Society-Faraday Transactions 92, 559-563, 1996b.

Taylor, R. S., Dang, L. X., and Garrett, B. C.: Molecular dynamics simulations of the liquid/vapor interface of SPC/E water, J. Phys. Chem., 100, 11720-11725, 1996.

Taylor, R. S., Ray, D., and Garrett, B. C.: Understanding the mechanism for the mass accommodation of ethanol by a water droplet, J. Phys. Chem. B, 101, 5473-5476, 1997.

Taylor, R. S. and Garrett, B. C.: Accommodation of alcohols by the liquid/vapor interface of water: Molecular dynamics study, J. Phys. Chem. B, 103, 844-851, 1999.

Taylor, R. S. and Shields, R. L.: Molecular-dynamics simulations of the ethanol liquid-vapor interface, J. Chem. Phys., 119, 1256912576, 2003.

Tervahattu, H, Hartonen, K, Kerminen, VM, Kupiainen, K, Aarnio, P; Koskentalo, T, Tuck, AF and Vaida, V,: New evidence of an organic layer on marine aerosols:, J. Geophys. Res. 107(D7), 4053, doi:10.1029/2000JD000282, 2002.

Thibert, E. and Dominé, F.: Thermodynamics and Kinetics of the Solid Solution of $\mathrm{HNO}_{3}$ in Ice. J. Phys. Chem. B, 102, 44324439, 1998.

Thomas, E. R., Frost, G. J., and Rudich, Y.: Reactive uptake of ozone by proxies for organic aerosols: Surface-bound and gas-phase products, J. Geophys. Res.-Atmos., 106, 3045-3056, 2001.

Thornberry, T. and Abbatt, J. P. D.: Heterogeneous reaction of ozone with liquid unsaturated fatty acids: detailed kinetics and gas-phase product studies, Phys. Chem. Chem. Phys., 6, 84-93,
2004.

Thornberry, T. D. and Abbatt, J. P. D.: Detailed analysis of the kinetics and products of the reaction of ozone with condensed phase oleic acid, Abstracts of Papers Am. Chem. Soc., 224, U328-U328, 2002.

Thornton, J. A. and Abbatt, J. P. D.: $\mathrm{N}_{2} \mathrm{O}_{5}$ reaction on sub-micron sea-salt aerosols: Kinetics, products and the effect of surface active organics, J. Phys. Chem. A., 109, 10004-10012, 2005 a.

Thornton, J. A. and Abbatt, J. P. D.: Measurements of $\mathrm{HO}_{2}$ uptake to aqueous aerosol: Mass accommodation coefficients and net reactive loss, J. Geophys. Res., 110, D08309, doi:10.1029/2004JD005402, 2005b.

Thornton, J. A., Braban, C. F., and Abbatt, J. P. D.: $\mathrm{N}_{2} \mathrm{O}_{5}$ hydrolysis on sub-micron organic aerosols: the effect of relative humidity, particle phase, and particle size, Phys. Chem. Chem. Phys., 5, 4593-4603, 2003.

Townsend, R. M., Gryko, J., and Rice, S. A.: Structure of the Liquid Vapor Interface of Water, J. Chem. Phys., 82, 4391-4392, 1985.

Tsuruta, T. and Nagayama, G.: Molecular dynamics studies on the condensation coefficient of water, J. Phys. Chem. B., 108, 1736$1743,2004$.

Ullerstam, M., Vogt, R., Langer, S., and Ljungström, E.: The kinetics and mechanism of $\mathrm{SO}_{2}$ oxidation by $\mathrm{O}_{3}$ on mineral dust, Phys. Chem. Chem. Phys., 4, 4694-4699, 2002.

Ullerstam, M., Thornberry, T., and Abbatt, J. P. D.: Uptake of gasphase nitric acid to ice at low partial pressures: Evidence for unsaturated surface coverage, Faraday Discuss., 130, 211-226, 2005.

Ullerstam, M., Johnson, M. S., Vogt, R., and Ljungström, E.: DRIFTS and Knudsen cell study of the heterogeneous reactivity of $\mathrm{SO}_{2}$ and $\mathrm{NO}_{2}$ on mineral dust, Atmos. Chem. Phys., 3, 2043-2051, doi:10.5194/acp-3-2043-2003, 2003.

Underwood, G. M., Li, P., Al-Abadleh, H., and Grassian, V. H.: A Knudsen cell study of the heterogeneous reactivity of nitric acid on oxide and mineral dust particles, J. Phys. Chem. A, 105, 6609-6620, 2001.

Usher, C. R., Michel, A. E., and Grassian, V. H.: Reactions on mineral dust, Chem. Rev., 103, 4883-4939, 2003.

Utter, R. G., Burkholder, J. B., Howard, C. J., and Ravishankara, A. R.: Measurement of the Mass Accommodation Coefficient of Ozone on Aqueous Surfaces, J. Phys. Chem., 96, 4973-4979, 1992.

Vacha, R., Slavicek, P., Mucha, M., Finlayson-Pitts, B. J., and Jungwirth, P.: Adsorption of atmospherically relevant gases at the air/water interface: Free energy profiles of aqueous solvation of $\mathrm{N}_{2}, \mathrm{O}_{2}, \mathrm{O}_{3}, \mathrm{OH}, \mathrm{H}_{2} \mathrm{O}, \mathrm{HO}_{2}$, and $\mathrm{H}_{2} \mathrm{O}_{2}$, J. Phys. Chem. A, 108, 11573-11579, 2004.

Vazquez, G., Alvarez, E., Cancela, A., and Navaza, J. M.: Density, Viscosity, and Surface-Tension of Aqueous-Solutions of Sodium-Sulfite and Sodium-Sulfite Plus Sucrose from 25Degrees-C to 40-Degrees-C, J. Chem. Eng. Data, 40, 1101$1105,1995$.

Vesala, T., Kulmala, M., Rudolf, R., Vrtala, A., and Wagner, P. E.: Models for condensational growth and evaporation of binary aerosol particles, J. Aerosol Sci., 28, 565-598, 1997.

Vieceli, J., Roeselova, M., and Tobias, D. J.: Accommodation coefficients for water vapor at the air/water interface, Chem. Phys. Lett., 393, 249-255, 2004.

Vieceli, J., Roeselova, M., Potter, N., Dang, L. X., Garrett, B. 
C., and Tobias, D. J.: Molecular dynamics simulations of atmospheric oxidants at the air-water interface: Solvation and accommodation of $\mathrm{OH}$ and $\mathrm{O}_{3}$, J. Phys. Chem. B., 109, 15876-15892, 2005.

Vlasenko, A., George, I. J., and Abbatt, J. P. D.: Formation of Volatile Organic Compounds in the Heterogeneous Oxidation of Condensed-Phase Organic Films by Gas-Phase OH, J. Phys. Chem. A, 112, 1552-1560, 2008.

Vlasenko, A., Huthwelker, T., Gaggeler, H. W., and Ammann, M.: Kinetics of the heterogeneous reaction of nitric acid with mineral dust particles: an aerosol flowtube study, Phys. Chem. Chem. Phys., 11, 7921-7930, 2009.

Vlasenko, A., Sjogren, S., Weingartner, E., Stemmler, K., Gäggeler, H. W., and Ammann, M.: Effect of humidity on nitric acid uptake to mineral dust aerosol particles, Atmos. Chem. Phys., 6, 21472160, doi:10.5194/acp-6-2147-2006, 2006.

Voigtländer, J., Stratmann, F., Niedermeier, D., Wex, H., and Kiselev, A.: Mass Accommodation coefficient of water: A combined computational fluid dynamics and experimental analysis, J. Geophys. Res., 112, D2028, doi:10.1029/2007JD008604, 2007.

Wachsmuth, M., Gäggeler, H. W., von Glasow, R., and Ammann, M.: Accommodation coefficient of HOBr on deliquescent $\mathrm{NaBr}$ aerosol particles, Atmos. Chem. Phys., 2, 121-131, doi:10.5194/acp-2-121-2002, 2002.

Wadia, Y., Tobias, D. J., Stafford, R., and Finlayson-Pitts, B. J.: Real-time monitoring of the kinetics and gas-phase products of the reaction of ozone with an unsaturated phospholipid at the airwater interface, Langmuir, 16, 9321-9330, 2000.

Wagner, C., Hanisch, F., Holmes, N., de Coninck, H., Schuster, G. and Crowley, J. N.: The interaction of $\mathrm{N}_{2} \mathrm{O}_{5}$ with mineral dust: aerosol flow tube and Knudsen reactor studies, Atmos. Chem. Phys., 8, 91-109, doi:10.5194/acp-8-91-2008, 2008.

Wagner, C., Schuster, G., and Crowley, J. N.: An aerosol flow tube study of the interaction of $\mathrm{N}_{2} \mathrm{O}_{5}$ with calcite, Arizona dust and quartz, Atmos. Environ., 43, 5001-5008, 2009.

Wagner, P. E.: A constant-angle Mie scattering method (CAMS) for investigation of particle formation processes, J. Coll. Interf. Sci., 105, 456-467, 1985.

Wahner, A., Mentel, T. F., Sohn, M., and Stier, J.: Heterogeneous reaction of $\mathrm{N}_{2} \mathrm{O}_{5}$ on sodium nitrate aerosol, J. Geophys. Res., 193, 31103-31112, doi:10.1029/1998JD100022, 1998.

Walser, M. L., Park, J., Gomez, A. L., Russell, A. R., and Nizkorodov, S.: Photochemical aging of secondary organic aerosol particles generated from the oxidation of d-limonene, Phys. Chem. A, 111, 1907-1913, 2007.

Walser, M. L., Desyaterik, Y., Laskin, J., Laskin, A., and Nizkorodov, S.: High-resolution mass spectrometric analysis of secondary organic aerosol produced by ozonation of limonene, Phys. Chem. Chem. Phys., 10, 1009-1022, 2008.

Wei, X., Miranda, P. B., and Shen, Y. R.: Surface vibrational spectroscopic study of surface melting ice, Phys. Rev. Lett. , 86, 1554-1557, 2001.

Wilson, M., Pohorille, A., and Pratt, L. R.: Molecular-Dynamics of the Water Liquid Vapor Interface, J. Phys. Chem. , 91, 48734878, 1987a.

Wilson, M. A., Pohorille, A., and Pratt, L. R.: Molecular-Dynamics of the Water Liquid-Vapor Interface, Journal of the Electrochemical Society, 134, C505-C505, 1987b.
Wilson, M. A. and Pohorille, A.: Adsorption and solvation of ethanol at the water liquid-vapor interface: A molecular dynamics study, J. Phys. Chem. B, 101, 3130-3135, 1997.

Winkler, A. K., Holmes, N. S., Crowley, J. N., et al.: Interaction of methanol, acetone, and formaldehyde with ice surfaces between 198 and 223 K, Phys. Chem. Chem. Phys., 4, 5270-5275, 2002.

Winkler, P. M., Vrtala, A., Wagner, P. E., Kulmala, M., Lehtinen, K. E. J., and Vesala, T.: Mass and thermal accommodation during gas-liquid condensation of water, Phys. Rev. Lett., 93, 075701, doi:10.1103/PhysRevLett.93.075701, 2004.

Winkler, P.M., Vrtala, A., Rudolf, R., Wagner, P. E., Riipinen, I., Vesala, T., Lehitnen, K. E. J., Viisanen, Y., and Kulmala, M.: Condensation of water vapor: Experimental determination of mass and thermal accommodation coefficients, J. Geophys. Res., 111, D19202, doi:10.1029/2006JD007194, 2006.

Wooldridge, P., Zhang, R., and Molina, M. J.: Phase equilibria of $\mathrm{H}_{2} \mathrm{SO}_{4}, \mathrm{HNO}_{3}$ and $\mathrm{HCl}$ hydrates and the composition of polar stratospheric clouds, J. Geophys. Res., 100, 1389-1396, 1995.

Worsnop, D. R., Zahniser, M. S., Kolb, C. E., Gardner, J. A., Watson, L. R., Van Doren, J. M., Jayne, J. T., and Davidovits, P.: Temperature dependence of mass accommodation of sulfur dioxide and hydrogen peroxide on aqueous surfaces, J. Phys. Chem., 93, 1159-1172, 1989.

Worsnop, D. R., Fox, L. E., Zahniser, M. S., and Wofsy, S. C.: Vapor pressures of solid hydrates of nitric acid: implications for polar stratospheric clouds, Science, 259, 71-74, 1993.

Worsnop, D. R., Shi Q., Jayne, J. T., Kolb, C. E., Swartz, E., and Davidovits, P.: Gas Phase Diffusion in Droplet Train Measurements of Uptake Coefficients, J. Aerosol Sci., 32, 877-881, 2001.

Worsnop, D. R., Williams, L. R., Kolb, C. E., Mozurkewich, M., Gershenzon, M., and Davidovits, P.: Comment on "The $\mathrm{NH}_{3}$ mass accommodation coefficient for uptake onto sulfuric acid solution”, J. Phys. Chem. A., 108, 8546-8548, 2004 a.

Worsnop, D. R., Williams, L. R., Kolb, C. E., Mozurkewich, M., Gershenzon, M., and Davidovits, P.: Comment on "Gas-phase flow and diffusion analysis of the droplet-train/flow-reactor technique for the mass accommodation process, J. Phys. Chem. A, 108: 8542-8543, 2004b.

Wren, S. N. and Donaldson, D. J.: Glancing-Angle Raman Spectroscopic Probe for Reaction Kinetics at Water Surfaces, Phys. Chem. Chem. Phys., 12, 2648-2654, 2010.

Zafiriou, O. C., Joussotdubien, J., Zepp, R. G., and Zika, R. G.: Photochemistry of Natural-Waters, Environ. Sci. Technol., 18, A358-A371, 1984.

Zhu, S. B., Fillingim, T. G., and Robinson, G. W.: Flexible Simple Point-Charge Water In A Self-Supporting Thin-Film, J. Phys. Chem., 95, 1002-1006, 1991.

Ziemann, P. J.: Aerosol products, mechanisms, and kinetics of heterogeneous reactions of ozone with oleic acid in pure and mixed particles, Faraday Discussions, 130, 469-490, 2005.

Zientara, M., Jakubczyk, D., Kolwas, K., and Kolwas, M.: Temperature Dependence of the Evaporation Coefficient of Water in Air and Nitrogen under Atmospheric Pressure: Study in Water Droplets, J. Phys. Chem. A, 112, 5152-5158, 2008.

Zondlo, M. A., Hudson, P. K., Prenni, A. J., and Tolbert, M. A.: Chemistry and microphysics of polar stratospheric clouds and Cirrus clouds, Ann. Rev. Phys. Chem., 51, 473-499, 2000. 